\title{
Further improvements in Waring's problem, IV: Higher powers
}

by

\author{
R. C. Vaughan (University Park, PA) and \\ T. D. Wooley (Ann Arbor, MI)
}

1. Introduction. As usual we define $G(k)$ to be the least number $s$ such that every sufficiently large natural number is the sum of, at most, $s k$ th powers of natural numbers. In this paper we continue the program, initiated in Vaughan and Wooley [18] and extended in [16] and [17], of comprehensively developing the repeated efficient differencing process of Wooley [19]. Following Vaughan [13], our methods depend on upper bounds for the number, $S_{s}^{(k)}(P, R)$, of solutions of the diophantine equations

$$
x_{1}^{k}+\ldots+x_{s}^{k}=y_{1}^{k}+\ldots+y_{s}^{k},
$$

with $x_{i}, y_{i} \in \mathcal{A}(P, R)$, where throughout we write

$$
\mathcal{A}(P, R)=\{n \in[1, P] \cap \mathbb{Z}: p \text { prime, } p \mid n \text { implies } p \leq R\} .
$$

In Vaughan and Wooley [18] we established bounds for $G(k)$ when $5 \leq k \leq 9$, and reported on preliminary bounds for $G(k)$ when $10 \leq k \leq 15$. We now extend the latter calculations to bound $G(k)$ when $9 \leq k \leq 20$, exploiting subsequent developments and making some further technical refinements.

Theorem 1.1. When $9 \leq k \leq 20$, one has $G(k) \leq H(k)$, where $H(k)$ is given in the following table.

\begin{tabular}{c|cccccccccccc}
\hline$k$ & 9 & 10 & 11 & 12 & 13 & 14 & 15 & 16 & 17 & 18 & 19 & 20 \\
$H(k)$ & 50 & 59 & 67 & 76 & 84 & 92 & 100 & 109 & 117 & 125 & 134 & 142 \\
\hline
\end{tabular}

For comparison, Wooley $[19,22]$ has obtained the bounds $G(k) \leq 8 k-18$ for $10 \leq k \leq 17$, and $G(18) \leq 127, G(19) \leq 135, G(20) \leq 144$. Meanwhile,

2000 Mathematics Subject Classification: 11P05, 11J71, 11P55.

Research of the first author supported in part by NSF grant DMS-9970632.

Research of the second author supported in part by NSF grants DMS-9622773 and DMS-9970440, and by a Fellowship from the David and Lucile Packard Foundation. Much of this paper was completed during the author's stay at the Institute for Advanced Study and Princeton University, supported by the 1998 Salem Prize. 
for smaller exponents one has the bounds

$$
G(5) \leq 17, \quad G(6) \leq 24, \quad G(7) \leq 33, \quad G(8) \leq 42, \quad G(9) \leq 51,
$$

by collecting together the conclusions of Vaughan and Wooley [16, 17, 18]. Indeed, we reported in [18] that preliminary calculations indicated the validity of the bounds

$$
\begin{gathered}
G(10) \leq 59, \quad G(11) \leq 67, \quad G(12) \leq 76, \quad G(13) \leq 84, \\
G(14) \leq 92, \quad G(15) \leq 100,
\end{gathered}
$$

though we gave no details of these calculations. It is worth remarking that a number of authors have obtained estimates weaker than those established (or described) in Vaughan and Wooley [18] and Wooley [22] (see especially $[8,9,10,11])$, following the publication of the papers [18] and [22]. However, the only improvement on the bounds contained in [18] and [22] known to the authors is a result of Meng [11], namely that $G(20) \leq 143$, and this is now superseded by Theorem 1.1.

It transpires that our estimates for the mean values $S_{s}^{(k)}(P, R)$ required in the proof of Theorem 1.1 are also of use in both localised and unlocalised estimates for the fractional part of $\alpha n^{k}$.

Theorem 1.2. Let $\alpha \in \mathbb{R}$ and $\varepsilon>0$. Then when $7 \leq k \leq 20$, there is a real number $N(\varepsilon, k)$ with the property that whenever $N \geq N(\varepsilon, k)$, one has

$$
\min _{1 \leq n \leq N}\left\|\alpha n^{k}\right\| \leq N^{\varepsilon-\sigma(k)},
$$

where $\sigma(k)^{-1}=S(k)$, and $S(k)$ is given by the following table.

\begin{tabular}{c|ccccccc}
\hline$k$ & 7 & 8 & 9 & 10 & 11 & 12 & 13 \\
$S(k)$ & 57.23 & 69.66 & 82.08 & 94.62 & 107.27 & 119.78 & 132.34 \\
\hline \multicolumn{10}{c}{} \\
\hline$k$ & 14 & 15 & 16 & 17 & 18 & 19 & 20 \\
$S(k)$ & 145.02 & 157.76 & 170.52 & 183.32 & 196.24 & 209.17 & 222.16 \\
\hline
\end{tabular}

For comparison, Baker [1] shows that $\sigma(k)^{-1}=2^{k-1}$ is permissible in Theorem 1.2 for each $k$ (following Danicic [5]), and describes how Vinogradov's methods yield sharper estimates for larger $k$. We note that Theorem 1.2 provides improvements on these exponents whenever $k \geq 7$. When $k$ is large, meanwhile, the conclusion of Theorem 1.2 of Wooley [22] shows that $\sigma(k)^{-1}=k(\log k+O(\log \log k))$ is permissible.

Theorem 1.3. Let $\alpha \in \mathbb{R}$ and $\varepsilon>0$. Then there are infinitely many natural numbers $n$ with

$$
\left\|\alpha n^{k}\right\| \leq n^{\varepsilon-\tau(k)},
$$


where $\tau(k)^{-1}=T(k)$, and $T(k)$ is given by the following table.

\begin{tabular}{c|ccccccc}
\hline$k$ & 8 & 9 & 10 & 11 & 12 & 13 & 14 \\
$T(k)$ & 57.72 & 67.25 & 76.71 & 86.18 & 95.46 & 104.77 & 114.02 \\
\hline \multicolumn{10}{|c}{} \\
\hline$k$ & 15 & 16 & 17 & 18 & 19 & 20 \\
$T(k)$ & 123.24 & 132.46 & 141.64 & 150.82 & 159.95 & 169.06 \\
\hline
\end{tabular}

The conclusion of Theorem 1.3 may be compared with Corollary 2 to Theorem 1.1 of Wooley [20], which shows that the exponent $\tau(k)^{-1}=$ $9.028 k$ is permissible for every $k$ (improving on earlier work of Heath-Brown [7]). For smaller $k$, moreover, Heath-Brown [7] has shown that $\tau(k)^{-1}=$ $3 \cdot 2^{k-3}$ is permissible for $k \geq 6$. The conclusion of Theorem 1.3 improves on the latter for $k \geq 8$. For $k=7$, meanwhile, our methods yield $\tau(7)^{-1}=$ 48.13, which narrowly fails to surpass Heath-Brown's exponent $\tau(7)^{-1}=48$. As noted by Heath-Brown [7], when $\alpha$ is algebraic, the method used to establish Theorem 1.3 shows, via an application of Roth's theorem, that the conclusion of Theorem 1.2 holds with $\sigma(k)$ replaced by $\tau(k)$.

Broadly speaking, our proof of Theorem 1.1 follows the pattern of Vaughan and Wooley [18]. We discuss the salient features of the underlying methods in Section 2 of this paper. The calculations involved in the proof are substantial, and thus one of the major challenges of this paper is the development of a strategy for handling the inherent complexity of our methods. There are three significant improvements on the methods of [18] of which we make use. Firstly, we employ the methods of Vaughan and Wooley [16], together with some refinements described in Section 5, in order to better handle the mean values of exponential sums over difference polynomials on the major arcs of our Hardy-Littlewood dissection. Such methods significantly enhance our estimates for mean values towards the end of the iteration process. Secondly, we make use of the new estimates for smooth Weyl sums contained in Wooley [22]. For larger $k$, these new estimates alone save several variables in the representations underlying our bounds for $G(k)$. Finally, in Section 3 of this paper, we establish new estimates for mean values of $2^{l}$ th power moments of exponential sums over difference polynomials, establishing an important technical refinement of the corresponding estimates contained in [18]. Although these latter estimates are of significance only in the initial segment of the iteration process, they nonetheless lead to improvements in mean value estimates significant to the estimates recorded in Theorems 1.2 and 1.3. We remark that the highly technical estimates described in Wooley [21] offer the prospect of further refinements in the mean value estimates described herein. However, it would seem that for larger $k$, such improvements are not significant so far as bounds for $G(k)$ are concerned. 
2. Preliminary observations. In order to put the work of the present paper in its proper setting, we first recall some of the notation and discussion of [18]. Throughout, $k$ will denote an arbitrary integer exceeding 2 , the letter $s$ will denote a positive integer, and $\varepsilon$ and $\eta$ will denote sufficiently small positive numbers. We take $P$ to be a large real number depending at most on $k, s, \varepsilon$ and $\eta$. We use $\ll$ and $\gg$ to denote Vinogradov's well-known notation, implicit constants depending at most on $k, s, \varepsilon$ and $\eta$. We make frequent use of vector notation for brevity. For example, $\left(c_{1}, \ldots, c_{t}\right)$ is abbreviated to $\mathbf{c}$. Also, we write $e(\alpha)$ for $e^{2 \pi i \alpha}$, and $[x]$ for the greatest integer not exceeding $x$.

In an effort to simplify our analysis, we adopt the following convention concerning the numbers $\varepsilon$ and $R$. Whenever $\varepsilon$ or $R$ appear in a statement, either implicitly or explicitly, we assert that for each $\varepsilon>0$, there exists a positive number $\eta_{0}(\varepsilon, s, k)$ such that the statement holds whenever $R=P^{\eta}$, with $0<\eta \leq \eta_{0}(\varepsilon, s, k)$. Note that the "value" of $\varepsilon$, and $\eta_{0}$, may change from statement to statement, and hence also the dependency of implicit constants on $\varepsilon$ and $\eta$. Notice that since our iterative methods will involve only a finite number of statements (depending at most on $k, s$ and $\varepsilon$ ), there is no danger of losing control of implicit constants through the successive changes implicit in our arguments. Finally, we use the symbol $\approx$ to indicate that constants and powers of $R$ and $P^{\varepsilon}$ are to be ignored.

For each $s \in \mathbb{N}$ we take $\phi_{i}=\phi_{i, s}(i=1, \ldots, k)$ to be real numbers, with $0 \leq \phi_{i} \leq 1 / k$, to be chosen later. We then take

$$
\begin{aligned}
P_{j} & =2^{j} P, & M_{j} & =P^{\phi_{j}}, \\
H_{j} & =P_{j} M_{j}^{-k}, & Q_{j} & =P_{j}\left(M_{1} \ldots M_{j}\right)^{-1} \quad(0 \leq j \leq k),
\end{aligned}
$$

and here, and throughout, the empty product is taken to be unity. We also write

$$
\widetilde{H}_{j}=\prod_{i=1}^{j} H_{i} \quad \text { and } \quad \widetilde{M}_{j}=\prod_{i=1}^{j} M_{i} R .
$$

We define the modified forward difference operator, $\Delta_{1}^{*}$, by

$$
\Delta_{1}^{*}(f(x) ; h ; m)=m^{-k}\left(f\left(x+h m^{k}\right)-f(x)\right),
$$

and define $\Delta_{j}^{*}$ recursively by

$$
\begin{aligned}
\Delta_{j+1}^{*}\left(f(x) ; h_{1}, \ldots,\right. & \left.h_{j+1} ; m_{1}, \ldots, m_{j+1}\right) \\
& =\Delta_{1}^{*}\left(\Delta_{j}^{*}\left(f(x) ; h_{1}, \ldots, h_{j} ; m_{1}, \ldots, m_{j}\right) ; h_{j+1} ; m_{j+1}\right) .
\end{aligned}
$$

We also adopt the convention that $\Delta_{0}^{*}(f(x) ; h ; m)=f(x)$.

For $0 \leq j \leq k$ let

$$
\Psi_{j}=\Psi_{j}\left(z ; h_{1}, \ldots, h_{j} ; m_{1}, \ldots, m_{j}\right)=\Delta_{j}^{*}\left(f(z) ; 2 h_{1}, \ldots, 2 h_{j} ; m_{1}, \ldots, m_{j}\right),
$$

where $f(z)=\left(z-h_{1} m_{1}^{k}-\ldots-h_{j} m_{j}^{k}\right)^{k}$. 
We write

$$
f_{j}(\alpha)=\sum_{x \in \mathcal{A}\left(Q_{j}, R\right)} e\left(\alpha x^{k}\right), \quad f_{j}^{+}(\alpha)=\sum_{\substack{x \in \mathcal{A}\left(Q_{j}, R\right) \\ x>\frac{1}{2} Q_{j} R^{-j}}} e\left(\alpha x^{k}\right)
$$

and

$$
g_{j}(\alpha)=\sum_{\frac{1}{2} Q_{j} R^{-j}<x \leq Q_{j}} e\left(\alpha x^{k}\right)
$$

and write also

$$
F_{j}(\alpha)=\sum_{z, \mathbf{h}, \mathbf{m}} e\left(\alpha \Psi_{j}(z ; \mathbf{h} ; \mathbf{m})\right),
$$

where the summation is over $z, \mathbf{h}, \mathbf{m}$ with

$$
1 \leq z \leq P_{j}, \quad M_{i}<m_{i} \leq M_{i} R, \quad m_{i} \in \mathcal{A}(P, R), \quad 1 \leq h_{i} \leq 2^{j-i} H_{i},
$$

for $1 \leq i \leq j$. We define $S_{s}^{(k)}(P, R)$ as in the introduction. Suppose that the real numbers $\lambda_{s}^{(k)}(1 \leq s<\infty)$ have the property that

$$
S_{s}^{(k)}(P, R) \ll P^{\lambda_{s}^{(k)}+\varepsilon} .
$$

Then we say that the $\lambda_{s}^{(k)}$ are permissible exponents. Such numbers certainly exist, since we may trivially take $\lambda_{s}^{(k)}=2 s$. Then for each $s$, we define the quantity $\Delta_{s}^{(k)}$ by

$$
\lambda_{s}^{(k)}=2 s-k+\Delta_{s}^{(k)} .
$$

When $\lambda_{s}^{(k)}$ is a permissible exponent, we say that $\Delta_{s}^{(k)}$ is an admissible exponent. When no confusion is possible, we suppress the superscript $k$.

The efficient differencing process which underlies our arguments is implicit in the following lemmata.

LEMMA 2.1. We have

$$
\int_{0}^{1}\left|F_{0}(\alpha)^{2} f_{0}(\alpha)^{2 s}\right| d \alpha \ll P^{\varepsilon} M_{1}^{2 s-1}\left(P M_{1} Q_{1}^{\lambda_{s}}+\int_{0}^{1}\left|F_{1}(\alpha) f_{1}(\alpha)^{2 s}\right| d \alpha\right) .
$$

Further, the inequality $(2.2)$ holds also when $f_{i}(\alpha)$ is replaced by $f_{i}^{+}(\alpha)$ for $i=0,1$.

Pr o of. The inequality (2.2) is immediate from Lemma 2.1 of [18]. Meanwhile, a consideration of the intermediate underlying diophantine equations reveals that the replacement of the exponential sums $f_{i}(\alpha)$ by $f_{i}^{+}(\alpha)$ $(i=0,1)$ is easily accomodated within the argument of the proofs of Lemmata 2.2 and 2.3 of Wooley [19], and thus the second conclusion of the lemma also follows with minimal effort. 

by

Following [18], we abbreviate an inequality of the form (2.2) symbolically

$$
F_{0}^{2} f_{0}^{2 s} \mapsto F_{1} f_{1}^{2 s}
$$

with a similar convention when $f_{i}$ is replaced by $f_{i}^{+}(i=0,1)$.

Lemma 2.2. Whenever $0<t<2 s$ and $1 \leq j \leq k-1$, we have

$$
\int_{0}^{1}\left|F_{j}(\alpha) f_{j}(\alpha)^{2 s}\right| d \alpha \ll P^{\varepsilon}\left(Q_{j}^{\lambda_{t}}\right)^{1 / 2}\left(\widetilde{H}_{j} \widetilde{M}_{j} M_{j+1}^{4 s-2 t-1} T_{j+1}\right)^{1 / 2},
$$

where

$$
T_{j+1}=P \widetilde{H}_{j} \widetilde{M}_{j+1} Q_{j+1}^{\lambda_{2 s-t}}+\int_{0}^{1}\left|F_{j+1}(\alpha) f_{j+1}(\alpha)^{4 s-2 t}\right| d \alpha .
$$

Further, the inequality $(2.3)$ holds also when $f_{i}(\alpha)$ is replaced by $f_{i}^{+}(\alpha)$ for $i=j, j+1$.

Pr o of. The first conclusion of the lemma is immediate from Lemma 2.2 of [18], on correcting a typographic error in the statement of the latter. The second conclusion of the lemma follows as in the proof of Lemma 2.2 of [18], on making use of Lemmata 2.3 and 3.1 of Wooley [19], the replacement of the exponential sum $f_{i}(\alpha)$ by $f_{i}^{+}(\alpha)(i=j, j+1)$ leading to minor cosmetic changes only.

Lemma 2.3. Whenever $0<t<2 s$ and $1 \leq j \leq k-1$, we have

$$
\int_{0}^{1}\left|F_{j}(\alpha) f_{j}^{+}(\alpha)^{2 s}\right| d \alpha \ll P^{\varepsilon}\left(Q_{j}^{\lambda_{t}}\right)^{1 / 2}\left(\widetilde{H}_{j} \widetilde{M}_{j} M_{j+1}^{4 s-2 t-1} \widetilde{T}_{j+1}\right)^{1 / 2}
$$

where

$$
\widetilde{T}_{j+1}=P \widetilde{H}_{j} \widetilde{M}_{j+1} Q_{j+1}^{\lambda_{2 s-t}}+\int_{0}^{1}\left|F_{j+1}(\alpha) g_{j+1}(\alpha)^{2} f_{j+1}(\alpha)^{4 s-2 t-2}\right| d \alpha .
$$

Proof. The proof is based on the use of Lemmata 2.3 and 3.1 of Wooley [19], in a manner similar to the proof of Lemma 2.2 of Vaughan and Wooley [18]. By applying Schwarz's inequality as in the proof of Lemma 2.2 of [18], we find that

$$
\begin{aligned}
\int_{0}^{1} \mid F_{j}(\alpha) f_{j}^{+} & (\alpha)^{2 s} \mid d \alpha \\
& \ll\left(\int_{0}^{1}\left|f_{j}^{+}(\alpha)\right|^{2 t} d \alpha\right)^{1 / 2}\left(\int_{0}^{1}\left|F_{j}(\alpha)^{2} f_{j}^{+}(\alpha)^{4 s-2 t}\right| d \alpha\right)^{1 / 2} .
\end{aligned}
$$

But on considering the underlying diophantine equations, 


$$
\int_{0}^{1}\left|f_{j}^{+}(\alpha)\right|^{2 t} d \alpha \leq \int_{0}^{1}\left|f_{j}(\alpha)\right|^{2 t} d \alpha \ll Q_{j}^{\lambda_{t}+\varepsilon},
$$

and by the argument of the proof of Lemmata 2.3 and 3.1 of [19], again noting that the replacement of $f_{i}(\alpha)$ by $f_{i}^{+}(\alpha)$ is easily accomodated, one finds that

$$
\begin{aligned}
\int_{0}^{1}\left|F_{j}(\alpha)^{2} f_{j}^{+}(\alpha)^{4 s-2 t}\right| d \alpha & \\
& \ll P^{\varepsilon} \widetilde{H}_{j} \widetilde{M}_{j} M_{j+1}^{4 s-2 t-1}\left(P \widetilde{H}_{j} \widetilde{M}_{j+1} Q_{j+1}^{\lambda_{2 s-t}}+T_{j+1}^{*}\right),
\end{aligned}
$$

where $T_{j+1}^{*}$ denotes the number of solutions of the diophantine equation

$$
\Psi_{j+1}(z ; \mathbf{h} ; \mathbf{m})+\sum_{i=1}^{2 s-t}\left(x_{2 i-1}^{k}-x_{2 i}^{k}\right)=0,
$$

with $z, \mathbf{h}, \mathbf{m}$ satisfying (2.1) for $1 \leq i \leq j+1$, and with $x_{i} \in \mathcal{A}\left(Q_{j+1}, R\right)$ and $x_{i}>\frac{1}{2} Q_{j+1} R^{-j-1}$ for $1 \leq i \leq 4 s-2 t$. Here we note that our summation conditions differ from those of (3.3) of [19] only by virtue of the notation defined above, and the latter condition on the $x_{i}$.

Let $T_{j+1}^{+}$denote the number of solutions of the diophantine equation (2.10) with $z, h_{i}, m_{i}$ satisfying (2.1) for $1 \leq i \leq j+1$, and with

$$
\frac{1}{2} Q_{j+1} R^{-j-1}<x_{1}, x_{2} \leq Q_{j+1} \quad \text { and } \quad x_{i} \in \mathcal{A}\left(Q_{j+1}, R\right) \quad(3 \leq i \leq 4 s-2 t) .
$$

Then it is evident that $T_{j+1}^{*} \leq T_{j+1}^{+}$, and moreover, on considering the underlying diophantine equations,

$$
T_{j+1}^{+}=\int_{0}^{1} F_{j+1}(\alpha)\left|g_{j+1}(\alpha)^{2} f_{j+1}(\alpha)^{4 s-2 t-2}\right| d \alpha \leq \widetilde{T}_{j+1} .
$$

The conclusion of the lemma therefore follows by combining (2.7)-(2.9). by

We abbreviate inequalities of the form (2.3) and (2.5) symbolically

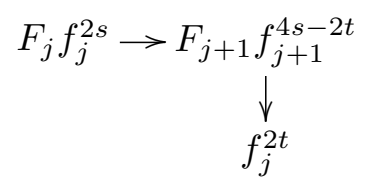

and

$$
\begin{gathered}
F_{j} f_{j}^{+2 s} \rightarrow F_{j+1} g_{j+1}^{2} f_{j+1}^{4 s-2 t-2} \\
\downarrow \\
f_{j}^{2 t}
\end{gathered}
$$

respectively. 
The integrals on the right hand side of (2.2), (2.4) and (2.6) may be estimated in two ways other than simply repeating the efficient differencing process.

Firstly, we may apply Hölder's inequality in the form

$$
\int_{0}^{1}\left|F_{j}(\alpha) f_{j}(\alpha)^{2 s}\right| d \alpha \ll I_{l}^{a} I_{l+1}^{b} U_{v}^{c} U_{w}^{d}
$$

where

$$
I_{m}=\int_{0}^{1}\left|F_{j}(\alpha)\right|^{2^{m}} d \alpha \quad(m=l, l+1)
$$

and

$$
U_{u}=\int_{0}^{1}\left|f_{j}(\alpha)\right|^{2 u} d \alpha \quad(u=v, w),
$$

in which $l, v$ and $w$ are non-negative integers, and $a, b, c, d$ are non-negative real numbers with

$$
a+b+c+d=1, \quad 2^{l} a+2^{l+1} b=1, \quad v c+w d=s .
$$

The $2^{m}$ th power mean values of $F_{j}$ may be estimated in terms of the number of solutions of certain diophantine equations, as we describe below. Also, we have $U_{v} \ll Q_{j}^{\lambda_{v}+\varepsilon}$ and $U_{w} \ll Q_{j}^{\lambda_{w}+\varepsilon}$. We abbreviate an inequality of the shape (2.11) symbolically by

$$
F_{j} f_{j}^{2 s} \Rightarrow\left(F_{j}^{2^{l}}\right)^{a}\left(F_{j}^{2^{l+1}}\right)^{b}\left(f_{j}^{2 v}\right)^{c}\left(f_{j}^{2 w}\right)^{d} .
$$

We discuss such inequalities further in Section 4 below.

Secondly, we may apply the Hardy-Littlewood method along the lines of Vaughan and Wooley [16]. We then abbreviate the resulting inequality symbolically in the form

$$
F_{j} f_{j}^{2 s} \Rightarrow\left(F_{j}\right)\left(f_{j}^{2 s}\right) \quad \text { or } \quad F_{j} g_{j}^{2} f_{j}^{2 s-2} \Rightarrow\left(F_{j}\right)\left(g_{j}^{2} f_{j}^{2 s-2}\right) .
$$

We discuss the material from [16] required in this paper in Section 5 below.

By considering the underlying diophantine equations, we have

$$
S_{s+1}(P, R) \leq \int_{0}^{1}\left|F_{0}(\alpha)^{2} f_{0}(\alpha)^{2 s}\right| d \alpha .
$$

Also, on writing

$$
H(\alpha ; Q)=\sum_{1 \leq x \leq Q} e\left(\alpha x^{k}\right) \text { and } h(\alpha ; Q)=\sum_{\substack{x \in \mathcal{A}(Q, R) \\ x>Q / 2}} e\left(\alpha x^{k}\right),
$$


it follows from a consideration of the underlying diophantine equations and Hölder's inequality that

$$
\begin{aligned}
S_{s+1}(P, R) & \leq \int_{0}^{1}\left|\sum_{\substack{i=0 \\
2^{i} \leq \sqrt{P}}}^{\infty} h\left(\alpha ; 2^{-i} P\right)+H(\alpha ; \sqrt{P})\right|^{2 s+2} d \alpha \\
& \ll P^{s+1}+(\log P)^{2 s+2} \max _{\substack{0 \leq i<\infty \\
2^{i} \leq \sqrt{P}}}^{1}\left|h\left(\alpha ; 2^{-i} P\right)\right|^{2 s+2} d \alpha \\
& \ll P^{s+1}+P^{\varepsilon} \max _{\substack{0 \leq i<\infty \\
2^{i} \leq \sqrt{P}}}^{1}\left|H\left(\alpha ; 2^{-i} P\right)^{2} h\left(\alpha ; 2^{-i} P\right)^{2 s}\right| d \alpha .
\end{aligned}
$$

Since the last integral has the shape

$$
\int_{0}^{1}\left|F_{0}(\alpha)^{2} f_{0}^{+}(\alpha)^{2 s}\right| d \alpha,
$$

it follows in either case that we may use a sequence of connected inequalities (in the obvious sense) to bound $S_{s}(Q, R)$ in terms of $S_{t}\left(Q^{\prime}, R\right)(t=1,2, \ldots)$. By optimising parameters one obtains in this way a permissible exponent $\lambda_{s+1}$ for which

$$
S_{s+1}(P, R) \ll P^{\lambda_{s+1}+\varepsilon} .
$$

The use of such inequalities within an iterative process is discussed in detail in Section 2 of [18]. While we avoid detailed discussion of such issues, we will indicate the manner in which optimal parameters are to be found. Notice that whenever the methods of this paper establish that $\lambda_{s+1}$ is a permissible exponent, then on considering the underlying diophantine equations, it is apparent that they also establish the upper bound

$$
\int_{0}^{1}\left|F_{0}(\alpha)^{2} f_{0}^{+}(\alpha)^{2 s}\right| d \alpha \ll P^{\lambda_{s+1}+\varepsilon},
$$

since our starting point in deriving such a permissible exponent is an application of Lemma 2.1. Either the mean value on the left hand side of (2.12) occurs explicitly in the latter application, or else a similar expression in which $f_{0}^{+}$is replaced by $f_{0}$, and of course a consideration of the underlying diophantine equations readily confirms that this last mean value majorises the former.

Finally, having established estimates for the mean values $S_{s}(P, R)$, one must still employ these bounds within the proofs of Theorems 1.1-1.3. We discuss the latter details in Sections 6 and 7. The calculation of the exponents $\lambda_{s}^{(k)}$, and subsequent computation of $G(k), \tau(k), \sigma(k)$ is deferred to Sections 8 to 23 . 
3. Estimates for the number of solutions of auxiliary equations. In this section we explore some technical refinements of the methods of Section 3 of [18] concerning the moments of the exponential sum $F_{j}(\alpha)$. Before proceeding further we require some notation. Let $R_{j}^{(s)}(P ; \phi)$ denote the number of solutions of the diophantine equation

$$
\sum_{i=1}^{s} \Psi_{j}\left(z_{i} ; \mathbf{h}^{(i)} ; \mathbf{m}^{(i)}\right)=\sum_{i=1}^{s} \Psi_{j}\left(w_{i} ; \mathbf{g}^{(i)} ; \mathbf{n}^{(i)}\right)
$$

with

$$
\begin{gathered}
1 \leq z_{i}, w_{i} \leq P_{j}, \quad 1 \leq h_{t}^{(i)}, g_{t}^{(i)} \leq 2^{j-t} H_{t}, \\
M_{t}<m_{t}^{(i)}, n_{t}^{(i)} \leq M_{t} R, \quad m_{t}^{(i)}, n_{t}^{(i)} \in \mathcal{A}(P, R),
\end{gathered}
$$

for $1 \leq t \leq j$ and $1 \leq i \leq s$. Also, we put $J=\left[\frac{1}{2}(k-j)\right]$, and define $K_{j}(P ; \phi)$ to be the number of solutions of the system of diophantine equations

$$
\sum_{i=1}^{j} h_{i}^{2 r}\left(m_{i}^{2 r k}-n_{i}^{2 r k}\right)=0 \quad(1 \leq r \leq J),
$$

with $\mathbf{h}, \mathbf{m}$ and $\mathbf{n}$ in the ranges defined by (3.2) and (3.3).

It transpires that the estimates for $R_{j}^{(s)}=R_{j}^{(s)}(P ; \phi)$ that are presently attainable are of interest only when $s$ is a small power of 2 . Moreover, one may bound $R_{j}^{\left(2^{l}\right)}$ in terms of $R_{j}^{\left(2^{l-1}\right)}$ and $R_{j+l}^{(1)}$ as follows.

Lemma 3.1. When $1 \leq l \leq k-2$ and $1 \leq j \leq k-l-1$, one has

$$
\begin{aligned}
& R_{j}^{\left(2^{l}\right)}\left(P ; \phi_{1}, \ldots, \phi_{j}\right) \\
& \ll P^{2^{l}-1}\left(\widetilde{H}_{j} \widetilde{M}_{j}\right)^{2^{l}} R_{j}^{\left(2^{l-1}\right)}\left(P ; \phi_{1}, \ldots, \phi_{j}\right) \\
& \quad+P^{2^{l+1}-2 l-2}\left(\widetilde{H}_{j} \widetilde{M}_{j}\right)^{2^{l+1}-2} R_{j+l}^{(1)}\left(P ; \phi_{1}, \ldots, \phi_{j}, 0, \ldots, 0\right) .
\end{aligned}
$$

Proof. This is a natural development of the proof of Lemma 3.1 of [18]. On considering the underlying diophantine equations, it follows from (3.1) that

$$
R_{j}^{\left(2^{l}\right)}(P ; \phi)=\int_{0}^{1}\left|F_{j}(\alpha)\right|^{2^{l+1}} d \alpha .
$$

Write

$$
\Psi_{j, l}(z ; \mathbf{h} ; \mathbf{m} ; \mathbf{u})=\Delta_{l}^{*}\left(\Psi_{j}(z ; \mathbf{h} ; \mathbf{m}) ; \mathbf{u} ; 1, \ldots, 1\right),
$$

where $\mathbf{u}=\left(u_{1}, \ldots, u_{l}\right)$. Then by applying standard Weyl differencing (see, for example, Lemma 2.3 of Vaughan [15]), an application of Hölder's inequality reveals that

$$
\left|F_{j}(\alpha)\right|^{2^{l}} \ll P^{2^{l}-1}\left(\widetilde{H}_{j} \widetilde{M}_{j}\right)^{2^{l}}+P^{2^{l}-l-1}\left(\widetilde{H}_{j} \widetilde{M}_{j}\right)^{2^{l}-1}|G(\alpha)|,
$$


where

$$
G(\alpha)=\sum_{\mathbf{h}, \mathbf{m}} \sum_{1 \leq u_{1} \leq P_{j}} \ldots \sum_{1 \leq u_{l} \leq P_{j}} \sum_{\substack{1 \leq z \leq P_{j}-u_{1}-\ldots-u_{l} \\ z \in I(\mathbf{u})}} e\left(\alpha \Psi_{j, l}(z ; \mathbf{h} ; \mathbf{m} ; \mathbf{u})\right),
$$

and here $I(\mathbf{u})$ denotes an interval depending only on $\mathbf{u}$, and the summation over $\mathbf{h}$ and $\mathbf{m}$ is over the ranges given in (3.2) and (3.3). Thus we deduce that

$$
\begin{aligned}
R_{j}^{\left(2^{l}\right)}(P ; \phi) \ll & P^{2^{l}-1}\left(\widetilde{H}_{j} \widetilde{M}_{j}\right)^{2^{l}} \int_{0}^{1}\left|F_{j}(\alpha)\right|^{2^{l}} d \alpha \\
& +P^{2^{l}-l-1}\left(\widetilde{H}_{j} \widetilde{M}_{j}\right)^{2^{l}-1} \int_{0}^{1}\left|G(\alpha) F_{j}(\alpha)^{2^{l}}\right| d \alpha .
\end{aligned}
$$

An application of Schwarz's inequality, combined with a consideration of the underlying diophantine equations, therefore reveals that

$$
\begin{aligned}
R_{j}^{\left(2^{l}\right)}(P ; \phi) \ll & P^{2^{l}-1}\left(\widetilde{H}_{j} \widetilde{M}_{j}\right)^{2^{l}} R_{j}^{\left(2^{l-1}\right)}(P ; \phi) \\
& +P^{2^{l}-l-1}\left(\widetilde{H}_{j} \widetilde{M}_{j}\right)^{2^{l}-1}\left(R_{j}^{\left(2^{l}\right)}(P ; \phi) S\right)^{1 / 2},
\end{aligned}
$$

where $S$ denotes the number of solutions of the equation

$$
\Psi_{j, l}(z ; \mathbf{h} ; \mathbf{m} ; \mathbf{u})=\Psi_{j, l}(w ; \mathbf{g} ; \mathbf{n} ; \mathbf{v}),
$$

with the variables $\mathbf{h}, \mathbf{g}, \mathbf{m}, \mathbf{n}$ in the ranges defined by (3.2) and (3.3), and with

$$
\begin{gathered}
1 \leq u_{i}, v_{i} \leq P_{j} \quad(1 \leq i \leq l), \\
1 \leq z \leq P_{j}-u_{1}-\ldots-u_{l}, \quad 1 \leq w \leq P_{j}-v_{1}-\ldots-v_{l} .
\end{gathered}
$$

But we have

$$
\begin{aligned}
2^{k} \Delta_{l}^{*}\left(\Psi_{j}(z ; \mathbf{h} ; \mathbf{m}) ; \mathbf{u} ; 1, \ldots, 1\right) & \\
& =\Delta_{j+l}^{*}\left(\left(2 z-2 h_{1} m_{1}^{k}-\ldots-2 h_{j} m_{j}^{k}\right)^{k} ; 4 \mathbf{h}, 2 \mathbf{u} ; \mathbf{m}, 1, \ldots, 1\right) \\
& =\Psi_{j+l}\left(2 z+u_{1}+\ldots+u_{l} ; 2 \mathbf{h}, \mathbf{u} ; \mathbf{m}, 1, \ldots, 1\right) .
\end{aligned}
$$

The desired conclusion therefore follows on noting that

$$
2 z+u_{1}+\ldots+u_{l}<2 P_{j} \leq P_{j+l} .
$$

The methods of Section 3 of [18] provide a bound of the shape

$$
R_{j}^{(1)}(P ; \phi) \ll P^{1+\varepsilon} K_{j}(P ; \phi)
$$

when $j=1$, and for $1 \leq j \leq k-2$ in circumstances in which $k-j$ is odd, and also when $k-j=2$ or 4 , but in all other circumstances the bounds obtained are somewhat unsatisfactory. Our primary aim in this section is to treat as many of the cases in which $k-j$ is even as is practicable. We handle the latter cases by making use of an estimate for the number of integral points 
on certain affine plane curves due to Bombieri and Pila [2] (this idea was mentioned to us in a conversation by Professor E. Bombieri in early 1991).

Lemma 3.2. Let $\mathcal{C}$ be the curve defined by the equation $F(x, y)=0$, where $F(x, y) \in \mathbb{R}[x, y]$ is an absolutely irreducible polynomial of degree $d \geq 2$. Also, let $N \geq \exp \left(d^{6}\right)$. Then the number of integral points on $\mathcal{C}$, and inside a square $[0, N] \times[0, N]$, does not exceed

$$
N^{1 / d} \exp \left(12(d \log N \log \log N)^{1 / 2}\right) .
$$

Proof. This is Theorem 5 of Bombieri and Pila [2].

Before announcing the new estimate at the heart of this discussion, we recall from Section 3 of [18] that for each $j$ and $k$ one has

$$
\Psi_{j}(z ; \mathbf{h} ; \mathbf{m})=k ! 2^{j} h_{1} \ldots h_{j} \sum_{u \geq 0} \sum_{v_{1} \geq 0} \ldots \sum_{v_{j} \geq 0} \frac{z^{u}\left(h_{1} m_{1}^{k}\right)^{2 v_{1}} \ldots\left(h_{j} m_{j}^{k}\right)^{2 v_{j}}}{u !\left(2 v_{1}+1\right) ! \ldots\left(2 v_{j}+1\right) !},
$$

where the summation is subject to the condition $u+2 v_{1}+\ldots+2 v_{j}=k-j$. Consequently, when $k-j$ is even, one has

$$
\Psi_{j}(z ; \mathbf{h} ; \mathbf{m})=h_{1} \ldots h_{j} \sum_{r=0}^{J} c_{r}\left(h_{1} m_{1}^{k}, \ldots, h_{j} m_{j}^{k}\right) z^{2 r},
$$

where the $c_{r}\left(\xi_{1}, \ldots, \xi_{j}\right) \in \mathbb{Z}[\boldsymbol{\xi}]$ are polynomials with positive coefficients which are symmetric in $\xi_{1}^{2}, \ldots, \xi_{j}^{2}$ of degree $J-r(0 \leq r \leq J)$.

Lemma 3.3. Suppose that $1 \leq j \leq k-6$ and $k-j$ is even. Then

$$
R_{j}^{(1)}(P ; \phi) \ll P^{1+\varepsilon} K_{j}(P ; \phi)+P^{2 / 3+\varepsilon} \widetilde{H}_{j} \widetilde{M}_{j}^{2} .
$$

Proof. Observe first that in view of $(3.5)$, the polynomial $\Psi_{j}(z ; \mathbf{h} ; \mathbf{m})$ is divisible by $h_{1} \ldots h_{j}$. The argument of the proof of Lemma 3.2 of [18] therefore shows that

$$
R_{j}^{(1)}(P ; \phi) \ll P^{\varepsilon} R_{j}^{*}(P ; \phi),
$$

where now we write $R_{j}^{*}(P ; \phi)$ for the number of solutions of the equation

$$
\Psi_{j}(z ; \mathbf{h} ; \mathbf{m})=\Psi_{j}(w ; \mathbf{h} ; \mathbf{n}),
$$

with $z, w, \mathbf{h}, \mathbf{m}, \mathbf{n}$ satisfying

$$
\begin{array}{r}
1 \leq z, w \leq P_{j}, \quad 1 \leq h_{i} \leq 2^{j-i} H_{i}, \quad m_{i}, n_{i} \in \mathcal{A}(P, R) \cap\left(M_{i}, M_{i} R\right] \\
(1 \leq i \leq j) .
\end{array}
$$

We divide our argument into cases. Let $R_{0}$ denote the number of solutions of the equation (3.7) counted by $R_{j}^{*}(P ; \phi)$ in which

$$
c_{0}\left(h_{1} m_{1}^{k}, \ldots, h_{j} m_{j}^{k}\right)=c_{0}\left(h_{1} n_{1}^{k}, \ldots, h_{j} n_{j}^{k}\right),
$$


and let $R_{1}$ denote the corresponding number of solutions in which (3.9) does not hold. Then one has

$$
R_{j}^{*}(P ; \phi)=R_{0}+R_{1} .
$$

Observe first that if $z, w, \mathbf{h}, \mathbf{m}, \mathbf{n}$ is any solution of (3.7) counted by $R_{0}$, then it follows from (3.5) and (3.9) that

$$
z^{2} \sum_{r=1}^{J} c_{r}\left(h_{1} m_{1}^{k}, \ldots, h_{j} m_{j}^{k}\right) z^{2 r-2}=w^{2} \sum_{r=1}^{J} c_{r}\left(h_{1} n_{1}^{k}, \ldots, h_{j} n_{j}^{k}\right) w^{2 r-2}
$$

When $t$ and $x$ are positive integers with $1 \leq x \leq P_{j}$, and $\mathbf{h}$ satisfies (3.8), denote by $r(n ; x, t ; \mathbf{h})$ the number of solutions of the simultaneous diophantine equations

$$
\begin{aligned}
x^{2} \sum_{r=1}^{J} c_{r}\left(h_{1} m_{1}^{k}, \ldots, h_{j} m_{j}^{k}\right) x^{2 r-2} & =n, \\
c_{0}\left(h_{1} m_{1}^{k}, \ldots, h_{j} m_{j}^{k}\right) & =t,
\end{aligned}
$$

with $\mathbf{m}$ satisfying (3.8). Then it follows from (3.11) via Cauchy's inequality and an elementary estimate for the divisor function that

$$
\begin{aligned}
R_{0} & =\sum_{n=1}^{\infty} \sum_{t=1}^{\infty} \sum_{\mathbf{h}}\left(\sum_{\substack{x^{2} \mid n \\
1 \leq x \leq P_{j}}} r(n ; x, t ; \mathbf{h})\right)^{2} \\
& \ll P^{\varepsilon} \sum_{n=1}^{\infty} \sum_{t=1}^{\infty} \sum_{\mathbf{h}} \sum_{\substack{x^{2} \mid n \\
1 \leq x \leq P_{j}}} r(n ; x, t ; \mathbf{h})^{2},
\end{aligned}
$$

where the summation over $\mathbf{h}$ is subject to (3.8). Thus it follows that

$$
R_{0} \ll P^{\varepsilon} R_{0}^{*},
$$

where $R_{0}^{*}$ denotes the number of solutions of the simultaneous diophantine equations

$$
\begin{array}{r}
\sum_{r=1}^{J}\left(c_{r}\left(h_{1} m_{1}^{k}, \ldots, h_{j} m_{j}^{k}\right)-c_{r}\left(h_{1} n_{1}^{k}, \ldots, h_{j} n_{j}^{k}\right)\right) z^{2 r-2}=0, \\
c_{0}\left(h_{1} m_{1}^{k}, \ldots, h_{j} m_{j}^{k}\right)-c_{0}\left(h_{1} n_{1}^{k}, \ldots, h_{j} n_{j}^{k}\right)=0,
\end{array}
$$

with $z, \mathbf{h}, \mathbf{m}, \mathbf{n}$ satisfying (3.8).

Consider next the solutions of the system (3.13), (3.14) in which

$$
c_{r}\left(h_{1} m_{1}^{k}, \ldots, h_{j} m_{j}^{k}\right) \neq c_{r}\left(h_{1} n_{1}^{k}, \ldots, h_{j} n_{j}^{k}\right)
$$

for some $r$ with $1 \leq r \leq J$. We may assign $\mathbf{h}, \mathbf{m}$ and $\mathbf{n}$ in $O\left(\widetilde{H}_{j} \widetilde{M}_{j}^{2}\right)$ ways. Fixing any one such choice, it follows that $z$ is determined by the non-trivial polynomial equation (3.13). Then there are $O(1)$ possible choices for $z$, and 
consequently the total number of solutions counted by $R_{0}^{*}$ of this type is $O\left(\widetilde{H}_{j} \widetilde{M}_{j}^{2}\right)$. The remaining solutions $z, \mathbf{h}, \mathbf{m}, \mathbf{n}$ of the system $(3.13),(3.14)$ counted by $R_{0}^{*}$ satisfy the system

$$
c_{r}\left(h_{1} m_{1}^{k}, \ldots, h_{j} m_{j}^{k}\right)=c_{r}\left(h_{1} n_{1}^{k}, \ldots, h_{j} n_{j}^{k}\right) \quad(0 \leq r \leq J),
$$

with the variable $z$ unconstrained. On recalling that the polynomials $c_{r}(\boldsymbol{\xi})$ have positive coefficients and are symmetric in $\xi_{1}^{2}, \ldots, \xi_{j}^{2}$ of degree $J-r$ $(0 \leq r \leq J)$, we find from (3.15) that the equations (3.4) are satisfied. Consequently, the number of possible choices for $\mathbf{h}, \mathbf{m}, \mathbf{n}$ is at most $K_{j}(P ; \boldsymbol{\phi})$. We may therefore conclude that

$$
R_{0}^{*} \ll \widetilde{H}_{j} \widetilde{M}_{j}^{2}+P K_{j}(P ; \phi) .
$$

On noting that the diagonal solutions of (3.4) alone yield $\gg \widetilde{H}_{j} \widetilde{M}_{j}$ solutions, and recalling that our hypotheses on $\phi$ dictate that $\widetilde{M}_{j} \ll P$, we find from (3.12) and (3.16) that

$$
R_{0} \ll P^{1+\varepsilon} K_{j}(P ; \phi) .
$$

Let $\mathbf{h}, \mathbf{m}, \mathbf{n}$ be any one of the $O\left(\widetilde{H}_{j} \widetilde{M}_{j}^{2}\right)$ possible choices satisfying (3.8) for which the equation (3.9) does not hold. Write

$$
F(x, y)=\sum_{r=0}^{J}\left(c_{r}\left(h_{1} m_{1}^{k}, \ldots, h_{j} m_{j}^{k}\right) x^{r}-c_{r}\left(h_{1} n_{1}^{k}, \ldots, h_{j} n_{j}^{k}\right) y^{r}\right) .
$$

Then it follows that for this fixed choice of $\mathbf{h}, \mathbf{m}, \mathbf{n}$, the choices of $z$ and $w$ to be counted by $R_{1}$ satisfy the equation

$$
F\left(z^{2}, w^{2}\right)=0
$$

with $1 \leq z, w \leq P_{j}$, and moreover the constant term in (3.18) is non-zero. Suppose first that the polynomial $F(x, y)$ is absolutely irreducible. Then it follows from Lemma 3.2 that the number of possible choices for $x$ and $y$ with $1 \leq x, y \leq P_{j}^{2}$, satisfying the equation $F(x, y)=0$, is $O\left(P^{2 / J+\varepsilon}\right)$. Hence the number of solutions of the equation (3.18) with $1 \leq z, w \leq P_{j}$ is similarly $O\left(P^{2 / J+\varepsilon}\right)$.

If, on the other hand, the polynomial $F(x, y)$ is not absolutely irreducible, then one may write $F(x, y)$ as a product of absolutely irreducible factors, say

$$
F(x, y)=\prod_{i=1}^{l} g_{i}(x, y) \prod_{e=1}^{m} h_{e}(x, y),
$$

where $l+m \geq 2$, and where $g_{i}(x, y) \in \mathbb{R}[x, y](1 \leq i \leq l)$, and

$$
h_{e}(x, y)=u_{e}(x, y)+v_{e}(x, y) \sqrt{-1} \quad(1 \leq e \leq m),
$$


with $u_{e}, v_{e} \in \mathbb{R}[x, y]$. We may suppose, moreover, that for each $e$ the polynomials $u_{e}$ and $v_{e}$ have no non-trivial polynomial common divisor over $\mathbb{C}[x, y]$. It therefore follows from Bezout's Theorem that the number of solutions of the simultaneous equations $u_{e}(x, y)=v_{e}(x, y)=0$ is bounded above by $J^{2}$. By considering real and imaginary components, therefore, the number of integral solutions of the equation $h_{e}(x, y)=0$ is also bounded above by $J^{2}$. If the degree of $g_{i}(x, y)$ exceeds 2 for any $i$, then the absolute irreducibility of $g_{i}(x, y)$ ensures, via Lemma 3.2, that the number of integral solutions of the equation $g_{i}(x, y)=0$, with $(x, y) \in\left[0, P_{j}^{2}\right]^{2} \cap \mathbb{Z}^{2}$, is $O\left(P^{2 / 3+\varepsilon}\right)$. Consequently, it follows from the conclusion of the previous paragraph together with (3.19) that the number of solutions of the equation (3.18) with $1 \leq z, w \leq P_{j}$ is $O\left(P^{2 / 3+\varepsilon}\right)$, except possibly when $F(x, y)$ factorises in the form (3.19) with one at least of the $g_{i}$ having degree one or two.

Suppose then that for some $i$ with $1 \leq i \leq l$, the polynomial $g_{i}(x, y)$ is quadratic or linear. If $g_{i}(x, y)$ is not some constant multiple of a $\mathbb{Q}$-rational polynomial, then since $g_{i}(x, y)$ is necessarily a constant multiple of a polynomial with algebraic coefficients, we deduce that the number of integral solutions of the equation $g_{i}(x, y)=0$ is at most $O(1)$. For we may remove the constant factor and consider components with respect to some basis for the field extension containing the coefficients of $g_{i}(x, y)$. Then since $g_{i}(x, y)$ is not a constant multiple of a $\mathbb{Q}$-rational polynomial, it follows that the integral zeros of the polynomial $g_{i}(x, y)$ necessarily satisfy at least two linearly independent $\mathbb{Q}$-rational equations, whence the desired conclusion follows from Bezout's Theorem.

Suppose next that for some $i$ with $1 \leq i \leq l$, the polynomial $g_{i}(x, y)$ is quadratic or linear, and has integral coefficients, as we may. Observe that the homogeneous part of $F(x, y)$ of maximal degree has the shape $\alpha\left(x^{J}-y^{J}\right)$, for a certain positive integer $\alpha$. Thus any quadratic factor of $F(x, y)$ must have homogeneous part of the shape $\alpha_{1} \phi(x, y)$, where $\alpha_{1}$ is rational and $\phi(x, y)$ is a divisor of $x^{J}-y^{J}$ with rational coefficients. By cyclotomy, the only possibilities for $\phi(x, y)$ are therefore $x^{2} \pm y^{2}$ and $x^{2} \pm x y+y^{2}$. Further, similarly, any linear factor of $F(x, y)$ must have homogeneous part of the shape $\alpha_{2}(x \pm y)$, where $\alpha_{2}$ is a rational number. In the latter case one sees that $g_{i}(x, y)$ has the shape $a(x \pm y)+c$, for a certain non-zero integer $a$, and an integer $c$. Moreover, since the constant term in (3.18) is non-zero, one has $c \neq 0$. But then the number of solutions $z, w$ of the equation (3.18), with $1 \leq z, w \leq P_{j}$, which arise from the vanishing of the factor $g_{i}$, is bounded above by the number of solutions of the equation

$$
a\left(z^{2} \pm w^{2}\right)+c=0
$$

with $1 \leq z, w \leq P_{j}$. But standard estimates for the number of solutions of such quadratic equations (see, for example, Estermann [6] or Lemma 3.5 of 
[18]) reveal that the number of solutions of the equation (3.20) counted by $R_{1}$ is at most $O\left(P^{\varepsilon}\right)$. If, on the other hand, the polynomial $g_{i}(x, y)$ is in fact a quadratic polynomial with rational coefficients, then in view of our earlier observation we may make a non-singular rational change of variables, $x=u+C_{1}, y=v+C_{2}$, so that the polynomial $g_{i}(x, y)$ takes the shape $a \phi(u, v)+c$ with $a$ and $c$ integers, and with $\phi(u, v)$ as above. The absolute irreducibility of $g_{i}(x, y)$, moreover, ensures that $a c \neq 0$. But then again the theory of binary quadratic equations ensures that the number of solutions of the equation $g_{i}\left(z^{2}, w^{2}\right)=0$, with $1 \leq z, w \leq P_{j}$, counted by $R_{1}$ is once more at most $O\left(P^{\varepsilon}\right)$.

Combining the conclusions of the previous four paragraphs, we find that for every fixed choice of $\mathbf{h}, \mathbf{m}, \mathbf{n}$ satisfying (3.8) for which the equation (3.9) does not hold, the number of possible choices for $z$ and $w$ satisfying (3.7) is at most $O\left(P^{2 / 3+\varepsilon}\right)$. Consequently,

$$
R_{1} \ll P^{2 / 3+\varepsilon} \widetilde{H}_{j} \widetilde{M}_{j}^{2}
$$

We therefore conclude from (3.6), (3.10), (3.17) and (3.21) that

$$
R_{j}^{(1)}(P ; \phi) \ll P^{1+\varepsilon} K_{j}(P ; \phi)+P^{2 / 3+\varepsilon} \widetilde{H}_{j} \widetilde{M}_{j}^{2},
$$

whence the lemma follows immediately.

On combining the conclusion of Lemma 3.3 with Lemma 3.1, we are able to obtain mean value estimates for $2^{l}$ th moments of $F_{j}(\alpha)$ which, in many circumstances, are superior to those available hitherto. We summarise such new estimates, and recall those previously known, in the following theorem.

Theorem 3.4. Suppose that $1 \leq l \leq k-2$ and $1 \leq j \leq k-1-l$. Let $\nu=[j / 2], J=\left[\frac{1}{2}(k-j-l+1)\right]$ and for $r \geq 1$ write $\delta_{r}=\lambda_{r}^{(2 J k)}-r$. Suppose that $\delta_{r}$ is increasing with $r$, and let e be 0 or 1 according to whether $j$ is even or odd. Finally, define the exponent $\sigma$ in general by taking $\sigma=\delta_{j} / j$, and when $\left(k+\delta_{2(\nu+f)}-2 \delta_{\nu+f}\right) \phi_{1} \leq 1(f=0, e)$, by taking $\sigma=\left(\delta_{\nu}+\delta_{\nu+e}\right) / j$. Then the following hold.

(Ia) Unconditionally, if $j=1$, or

(Ib) if any one of the following conditions hold:
( $\alpha) l=1$ and $k-j$ is either odd, or $k-j=2$ or 4 , or
( $\beta$ ) $l=2$ and $3 \leq k-j \leq 5$, or
$(\gamma) l=3$ and $k-j=4$ or 5 , or
( $\delta) \phi_{1}+\ldots+\phi_{j} \leq 1 / 3$,

and in addition any one of the following conditions also hold:

(i) $1 \leq j \leq J+1$, or

(ii) $2+e \leq j \leq 2 J+2-e$ and $\left(k+\delta_{j+e}\right) \phi_{1} \leq 1$, or 
(iii) when $j \geq 3$, we have

$$
\sum_{i=1}^{I} \phi_{i}+k\left(\phi_{I-1}+\phi_{I}\right) \leq 2 \quad(3 \leq I \leq j),
$$

then one has

$$
\int_{0}^{1}\left|F_{j}(\alpha)\right|^{2^{l}} d \alpha \ll P^{2^{l}-l+\varepsilon} \widetilde{M}_{j}^{2^{l}-1} \widetilde{H}_{j}^{2^{l}-1} .
$$

(Ic) If any one of the conditions $(\alpha),(\beta),(\gamma)$, or

$$
\left(\delta^{\prime}\right) \phi_{1}+\ldots+\phi_{j} \leq \frac{1}{3}(1-\sigma)^{-1}
$$

hold, and none of (i), (ii), (iii) hold, then one has

$$
\int_{0}^{1}\left|F_{j}(\alpha)\right|^{2^{l}} d \alpha \ll P^{2^{l}-l+\varepsilon} \widetilde{M}_{j}^{2^{l}-1+\sigma} \widetilde{H}_{j}^{2^{l}-1} .
$$

(II) If

(1) one of conditions (i), (ii), (iii) hold, and $\phi_{1}+\ldots+\phi_{j} \geq 1 / 3$, or

(2) none of (i), (ii), (iii) hold, and $\phi_{1}+\ldots+\phi_{j} \geq \frac{1}{3}(1-\sigma)^{-1}$,

then

$$
\int_{0}^{1}\left|F_{j}(\alpha)\right|^{2^{l}} d \alpha \ll P^{2^{l}-l-1 / 3+\varepsilon} \widetilde{M}_{j}^{2^{l}} \widetilde{H}_{j}^{2^{l}-1} .
$$

(III) In any case, one has

$$
\int_{0}^{1}\left|F_{j}(\alpha)\right|^{2^{l}} d \alpha \ll P^{2^{l}-l+\varepsilon} \widetilde{M}_{j}^{2^{l}} \widetilde{H}_{j}^{2^{l}-1} .
$$

Pr o of. For the sake of convenience, write $m=l-1$. We begin by noting that $M_{1} \leq P^{1 / 3}$ for $k \geq 3$, so that by combining the conclusions of Lemma 2.1 of Vaughan [14], Lemma 3.3 of [18], and Lemma 3.3 of the present paper, we conclude for $j=1$ that

$$
R_{j+r}^{(1)}(P ; \boldsymbol{\phi}, \mathbf{0}) \ll P^{1+\varepsilon}\left(P^{r} \widetilde{H}_{j}\right) \widetilde{M}_{j} \quad(0 \leq r \leq m) .
$$

Suppose next that one of the conditions (i), (ii) or (iii) holds. Then the argument of the proofs of Theorems 3.10 and 3.11 of [18] shows that

$$
K_{j+r}(P ; \boldsymbol{\phi}, \mathbf{0}) \ll P^{\varepsilon}\left(P^{r} \widetilde{H}_{j}\right) \widetilde{M}_{j} \quad(0 \leq r \leq m) .
$$

When conditions $(\alpha),(\beta)$ or $(\gamma)$ hold, it follows that for $0 \leq r \leq m$, the integer $k-j-r$ is either odd, or else is equal either to 2 or 4 . In these circumstances, Lemma 3.3 of [18] shows that

$$
R_{j+r}^{(1)}(P ; \boldsymbol{\phi}, \mathbf{0}) \ll P^{1+\varepsilon} K_{j+r}(P ; \boldsymbol{\phi}, \mathbf{0}) \quad(0 \leq r \leq m),
$$


whence the estimate (3.22) follows from (3.23). When condition $(\delta)$ holds, on the other hand, one has $\widetilde{M}_{j} \ll P^{1 / 3}$, and so we may conclude from Lemma 3.3 of the present paper together with Lemma 3.3 of [18] that for $0 \leq r \leq m$,

$$
\begin{aligned}
R_{j+r}^{(1)}(P ; \boldsymbol{\phi}, \mathbf{0}) & \ll P^{1+\varepsilon} K_{j+r}(P ; \boldsymbol{\phi}, \mathbf{0})+P^{2 / 3+\varepsilon}\left(P^{r} \widetilde{H}_{j}\right) \widetilde{M}_{j}^{2} \\
& \ll P^{1+\varepsilon} K_{j+r}(P ; \boldsymbol{\phi}, \mathbf{0})+P^{1+\varepsilon}\left(P^{r} \widetilde{H}_{j}\right) \widetilde{M}_{j} .
\end{aligned}
$$

Here we note that the former lemma applies when $k-j-r$ is an even integer exceeding 4 , and the latter when $k-j-r$ is odd, or equal to 2 or 4 . Thus the estimate (3.22) again follows from (3.23).

When none of the conditions (i), (ii), (iii) hold, meanwhile, then the argument of the proofs of Theorems 3.10 and 3.11 of [18] yields the estimate

$$
K_{j+r}(P ; \boldsymbol{\phi}, \mathbf{0}) \ll P^{r+\varepsilon} \widetilde{H}_{j} \widetilde{M}_{j}^{1+\sigma} .
$$

Since (3.24) again holds when conditions $(\alpha),(\beta)$ or $(\gamma)$ are satisfied, we deduce from (3.25) that when one of the latter conditions holds, one has

$$
R_{j+r}^{(1)}(P ; \boldsymbol{\phi}, \mathbf{0}) \ll P^{1+\varepsilon}\left(P^{r} \widetilde{H}_{j}\right) \widetilde{M}_{j}^{1+\sigma} \quad(0 \leq r \leq m) .
$$

When condition $\left(\delta^{\prime}\right)$ holds, meanwhile, one has $\widetilde{M}_{j}^{1-\sigma} \ll P^{1 / 3}$, and in such circumstances one may conclude from Lemma 3.3 of the present paper together with Lemma 3.3 of [18] that for $0 \leq r \leq m$,

$$
R_{j+r}^{(1)}(P ; \boldsymbol{\phi}, \mathbf{0}) \ll P^{1+\varepsilon} K_{j+r}(P ; \boldsymbol{\phi}, \mathbf{0})+P^{2 / 3+\varepsilon}\left(P^{r} \widetilde{H}_{j}\right)\left(\widetilde{M}_{j}^{1+\sigma} P^{1 / 3}\right),
$$

and hence (3.26) again follows from (3.25).

When condition (1) of the statement of Theorem 3.4 holds, one has $\widetilde{M}_{j} \gg P^{1 / 3}$, and thus from Lemma 3.3 of the present paper, Lemma 3.3 of [18], and (3.23), one obtains in a manner similar to that above,

$$
R_{j+r}^{(1)}(P ; \boldsymbol{\phi}, \mathbf{0}) \ll P^{2 / 3+\varepsilon}\left(P^{r} \widetilde{H}_{j}\right) \widetilde{M}_{j}^{2} \quad(0 \leq r \leq m) .
$$

When condition (2) of the statement of Theorem 3.4 holds, meanwhile, we have $\widetilde{M}_{j}^{1-\sigma} \gg P^{1 / 3}$, whence by Lemma 3.3 of the present paper, Lemma 3.3 of [18], and (3.25), one obtains the estimate (3.27) once again. Finally, we note that Lemma 3.2 of [18] provides the bound

$$
R_{j+r}^{(1)}(P ; \boldsymbol{\phi}, \mathbf{0}) \ll P^{1+\varepsilon}\left(P^{r} \widetilde{H}_{j}\right) \widetilde{M}_{j}^{2} \quad(0 \leq r \leq m) .
$$

On collecting together (3.22), (3.26) and (3.28), we find that in all cases one has a bound of the shape

$$
R_{j+r}^{(1)}(P ; \phi, \mathbf{0}) \ll P^{1+\varepsilon}\left(P^{r} \widetilde{H}_{j}\right) \widetilde{M}_{j}^{1+\tau} \quad(0 \leq r \leq m),
$$

where $\tau=0$ when conditions (Ia) or (Ib) hold, where $\tau=\sigma$ when (Ic) holds, where $\widetilde{M}_{j}^{\tau}=\widetilde{M}_{j} P^{-1 / 3}$ when (II) holds, and where $\tau=1$ when (III) holds. 
We now apply Lemma 3.1, obtaining from (3.29) for $1 \leq r \leq m$ the estimate

$$
\begin{aligned}
R_{j}^{\left(2^{r}\right)}(P ; \phi) \ll & P^{2^{r}-1}\left(\widetilde{H}_{j} \widetilde{M}_{j}\right)^{2^{r}} R_{j}^{\left(2^{r-1}\right)}(P ; \phi) \\
& +P^{2^{r+1}-2 r-2}\left(\widetilde{H}_{j} \widetilde{M}_{j}\right)^{2^{r+1}-2}\left(P^{r+1+\varepsilon} \widetilde{H}_{j} \widetilde{M}_{j}^{1+\tau}\right) \\
\ll & P^{2^{r+1}-r-1+\varepsilon} \widetilde{H}_{j}^{2^{r+1}-1} \widetilde{M}_{j}^{2^{r+1}-1+\tau} \\
& +P^{2^{r}-1}\left(\widetilde{H}_{j} \widetilde{M}_{j}\right)^{2^{r}} R_{j}^{\left(2^{r-1}\right)}(P ; \phi) .
\end{aligned}
$$

Then by inductively applying the formula (3.30), starting from the base

$$
R_{j}^{(1)}(P ; \phi) \ll P^{1+\varepsilon} \widetilde{H}_{j} \widetilde{M}_{j}^{1+\tau}
$$

supplied by (3.29), one deduces that for $0 \leq r \leq m$, one has

$$
R_{j}^{\left(2^{r}\right)}(P ; \phi) \ll P^{2^{r+1}-r-1+\varepsilon} \widetilde{H}_{j}^{2^{r+1}-1} \widetilde{M}_{j}^{2^{r+1}-1+\tau} .
$$

The conclusion of the theorem follows from the case $r=m$ of the latter formula, on considering the underlying diophantine equations.

For the sake of completeness we add a final mean value estimate related to those of Theorem 3.4 to our arsenal.

Theorem 3.5. Suppose that $2 \leq l \leq k-2$. Then

$$
\int_{0}^{1}\left|F_{k-l}(\alpha)\right|^{2^{l}} d \alpha \ll P^{2^{l}-l+\varepsilon} \widetilde{M}_{k-l}^{2^{l}} \widetilde{H}_{k-l}^{2^{l}-1} .
$$

Proof. On considering the underlying diophantine equations, the argument of the proof of case (III) of Theorem 3.4 leading to (3.29) yields

$$
R_{k-l+r}^{(1)}(P ; \boldsymbol{\phi}, \mathbf{0}) \ll P^{1+\varepsilon}\left(P^{r} \widetilde{H}_{k-l}\right) \widetilde{M}_{k-l}^{2} \quad(0 \leq r \leq l-2) .
$$

Moreover, it follows from (3.1) and the definition of $R_{k-1}^{(1)}(P ; \boldsymbol{\phi}, \mathbf{0})$ that

$$
R_{k-1}^{(1)}(P ; \phi, \mathbf{0}) \leq \widetilde{M}_{k-l}^{2} R^{*},
$$

where $R^{*}$ denotes the number of integral solutions of the equation

$$
z_{1} \ldots z_{l} h_{1} \ldots h_{k-l}=w_{1} \ldots w_{l} g_{1} \ldots g_{k-l}
$$

with $1 \leq z_{i}, w_{i} \leq P_{k-l}(1 \leq i \leq l)$ and $1 \leq h_{n}, g_{n} \leq 2^{k} H_{n}(1 \leq n \leq k-l)$. Let $\mathbf{z}, \mathbf{w}, \mathbf{g}, \mathbf{h}$ be a solution of (3.33) counted by $R^{*}$. Standard estimates for the divisor function reveal that for each fixed choice of $\mathbf{z}, \mathbf{h}$, one has $O\left(P^{\varepsilon}\right)$ possible choices for $\mathbf{w}, \mathbf{g}$, whence $R^{*}=O\left(P^{l+\varepsilon} \widetilde{H}_{k-l}\right)$. By (3.32), we therefore have

$$
R_{k-1}^{(1)}(P ; \boldsymbol{\phi}, \mathbf{0}) \ll P^{l+\varepsilon} \widetilde{M}_{k-l}^{2} \widetilde{H}_{k-l},
$$

so that (3.31) holds also when $r=l-1$. The lemma now follows by applying Lemma 3.1 inductively in the same manner as in the proof of Theorem 3.4. 
4. Iterative schemes based on mean value estimates. In our mean value based treatments we adopt two approaches, according to the situation. We consider below the consequences of estimates of the form

$$
\int_{0}^{1}\left|F_{j}(\alpha)\right|^{2^{l}} d \alpha \ll P^{2^{l}-l-\chi_{j, l}+\varepsilon} \widetilde{M}_{j}^{2^{l}-1+\tau_{j, l}} \widetilde{H}_{j}^{2^{l}-1},
$$

for a suitable $\tau_{j, l} \geq 0$, and $\chi_{j, l}=0$ or $1 / 3$. We suppose in what follows that $\lambda_{r}(r \in \mathbb{N})$ are known permissible exponents, and we seek a new permissible exponent $\lambda_{s}^{\prime}$.

(i) Process $A_{j}^{s, l}$. When $s \geq j$ we may adopt the scheme

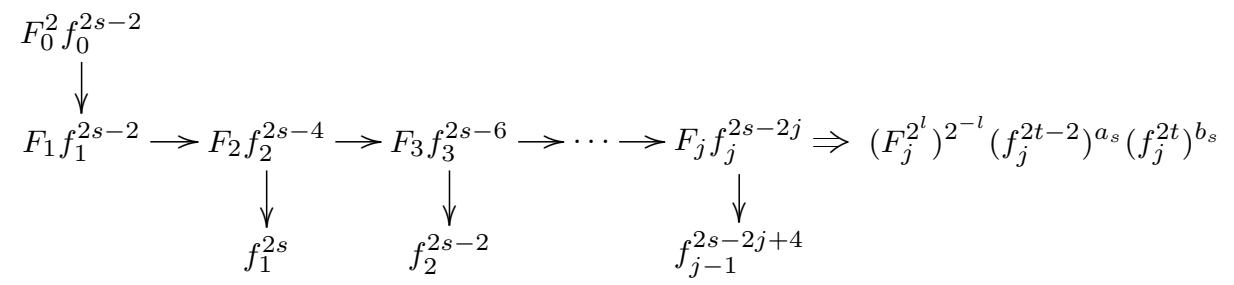

where $t, a_{s}$ and $b_{s}$ are defined by means of

$$
\begin{gathered}
t=\left[\left(1-2^{-l}\right)^{-1}(s-j)+1\right], \quad \theta=t-\left(1-2^{-l}\right)^{-1}(s-j), \\
a_{s}=\left(1-2^{-l}\right) \theta, \quad b_{s}=\left(1-2^{-l}\right)(1-\theta) .
\end{gathered}
$$

Following the argument of Section 2 of [18] (see also Section 11), and recalling the definitions of the parameters from Section 2, it follows from (4.1) that $\lambda_{s}^{\prime}$ and $\phi$ are determined by the equations

$$
\begin{aligned}
P \widetilde{H}_{j-1} \widetilde{M}_{j} Q_{j}^{\lambda_{s-j}} & \approx P^{1-\left(l+\chi_{j, l}\right) 2^{-l}} \widetilde{H}_{j}^{1-2^{-l}} \widetilde{M}_{j}^{1-2^{-l}\left(1-\tau_{j, l}\right)} Q_{j}^{a_{s} \lambda_{t-1}+b_{s} \lambda_{t}}, \\
P \widetilde{H}_{i-1} \widetilde{M}_{i} Q_{i}^{\lambda_{s-i}} & \approx\left(P\left(\widetilde{H}_{i} \widetilde{M}_{i}\right)^{2} M_{i+1}^{2(s-i-1)} Q_{i}^{\lambda_{s-i+1}} Q_{i+1}^{\lambda_{s-i-1}}\right)^{1 / 2} \\
P M_{1} Q_{1}^{\lambda_{s-1}} & \approx\left(P\left(H_{1} M_{1}\right)^{2} M_{2}^{2 s-4} Q_{1}^{\lambda_{s}} Q_{2}^{\lambda_{s-2}}\right)^{1 / 2}, \\
P^{\lambda_{s}^{\prime}} & \approx P M_{1}^{2 s-2} Q_{1}^{\lambda_{s-1}} .
\end{aligned}
$$

Here and throughout, we use the symbol $\approx$ to denote that factors involving $R$ and $P^{\varepsilon}$ to fixed powers are to be ignored.

Write

$$
\begin{aligned}
\delta & =\left(2^{l}-1\right)\left(\theta \lambda_{t-1}+(1-\theta) \lambda_{t}\right)-2^{l} \lambda_{s-j}, \\
\mathcal{E}_{i} & =\lambda_{s-i+1}-2 \lambda_{s-i}+\lambda_{s-i-1} \quad(1 \leq i<j), \\
\kappa_{i} & =2(s-i)-\lambda_{s-i} \quad(2 \leq i \leq j) .
\end{aligned}
$$


Now define $\alpha_{i}, \beta_{i}, \gamma_{i}$ for $1 \leq i \leq j$ by

$$
\begin{aligned}
\alpha_{j} & =\left(\left(2^{l}-1\right) k+\delta+1-\tau_{j, l}\right)^{-1}, \\
\beta_{j} & =-k+\delta+1-\tau_{j, l}, \\
\gamma_{j} & =2^{l}-l+\delta-j-\chi_{j, l},
\end{aligned}
$$

and for $i=j-1, \ldots, 1$, successively by

$$
\begin{aligned}
\gamma_{i} & =1+\mathcal{E}_{i}+\kappa_{i+1} \alpha_{i+1} \gamma_{i+1}, \\
\beta_{i} & =\mathcal{E}_{i}+\kappa_{i+1} \alpha_{i+1} \beta_{i+1}, \\
\alpha_{i} & =\left(2 k+\beta_{i}\right)^{-1} .
\end{aligned}
$$

Then on writing explicitly the equations relating the $\phi_{i}$ described above, and solving the resulting system of linear equations, one verifies with little difficulty that $\phi$ and $\lambda_{s}^{\prime}$ satisfy

$$
\begin{gathered}
\phi_{i}=\alpha_{i}\left(\gamma_{i}-\beta_{i}\left(\phi_{1}+\ldots+\phi_{i-1}\right)\right) \quad(2 \leq i \leq j), \\
\phi_{1}=\alpha_{1} \gamma_{1},
\end{gathered}
$$

and

$$
\lambda_{s}^{\prime}=\lambda_{s-1}\left(1-\phi_{1}\right)+1+(2 s-2) \phi_{1} .
$$

In this manner we may calculate a new permissible exponent $\lambda_{s}^{\prime}$, and in concert with other available iterative schemes we repeatedly derive new sequences $\left(\lambda_{r}\right)$ of permissible exponents, ultimately attaining an approximation to converged values (see the discussion of Section 2 of [18] for a detailed overview of such matters).

(ii) Process $B_{j, t}^{s, l}$. When $s \geq j$ and

$$
\left(1-2^{-l}\right)^{-1}(s-j) \leq t \leq\left(1-2^{1-l}\right)^{-1}(s-j),
$$

we may instead adopt the scheme

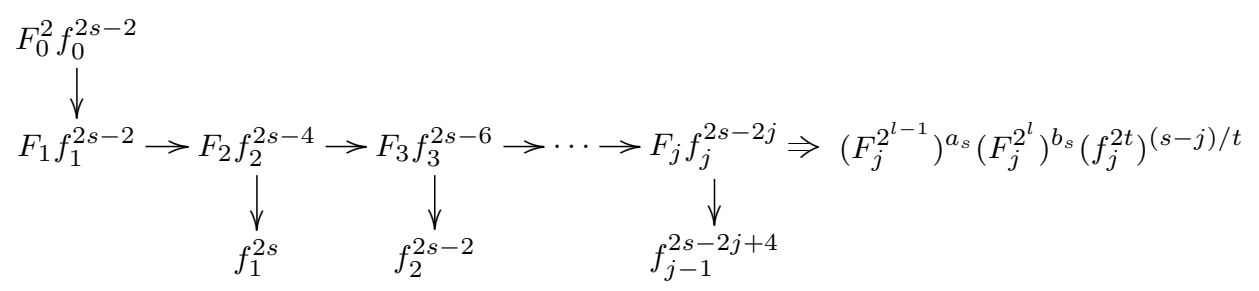

where $a_{s}$ and $b_{s}$ are defined by

$$
a_{s}=2-2^{1-l}-2(s-j) t^{-1}, \quad b_{s}=(s-j) t^{-1}-1+2^{1-l} .
$$

Following the argument of Section 2 of [18] (see also Section 11), it follows from (4.1) that $\lambda_{s}^{\prime}$ and $\phi$ are determined by the equations 


$$
\begin{aligned}
P \widetilde{H}_{j-1} \widetilde{M}_{j} Q_{j}^{\lambda_{s-j}} \approx & P^{\left(2^{l-1}-l+1-\chi_{j, l-1}\right) a_{s}+\left(2^{l}-l-\chi_{j, l}\right) b_{s}} \\
& \times\left(\widetilde{H}_{j} \widetilde{M}_{j}\right)^{\left(2^{l-1}-1\right) a_{s}+\left(2^{l}-1\right) b_{s}} \widetilde{M}_{j}^{a_{s} \tau_{j, l-1}+b_{s} \tau_{j, l}} Q_{j}^{\lambda_{t}(s-j) / t},
\end{aligned}
$$

and the equations (4.2)-(4.4).

We now write

$$
\delta=2(s-j) \lambda_{t}-2 t \lambda_{s-j},
$$

and define $\mathcal{E}_{i}$ and $\kappa_{i}$ as in (4.5) and (4.6). Also, we define in this case $\alpha_{i}$, $\beta_{i}, \gamma_{i}$ for $1 \leq i \leq j$ by means of

$$
\begin{aligned}
\alpha_{j} & =(2(s-j)(k-1)+2 t+\delta+\widetilde{\tau})^{-1}, \\
\beta_{j} & =2(k-1)(s-j-t)+\delta+\widetilde{\tau}, \\
\gamma_{j} & =2(j+l-2)(s-j-t)+t\left(2-2^{2-l}\right)+\delta-\widetilde{\chi},
\end{aligned}
$$

in which

$$
\begin{aligned}
& \tilde{\tau}=\left(4(s-j)-\left(4-2^{2-l}\right) t\right) \tau_{j, l-1}+\left(\left(2-2^{2-l}\right) t-2(s-j)\right) \tau_{j, l}, \\
& \tilde{\chi}=\left(\left(4-2^{2-l}\right) t-4(s-j)\right) \chi_{j, l-1}+\left(2(s-j)-t\left(2-2^{2-l}\right)\right) \chi_{j, l},
\end{aligned}
$$

and for $i=j-1, \ldots, 1$, successively by (4.7). Then we find once again that $\phi$ and $\lambda_{s}^{\prime}$ satisfy (4.8)-(4.10), and once more we are able to establish new permissible exponents by iterating this and allied procedures.

Notice that both processes $A_{j}^{s, l}$ and $B_{j, t}^{s, l}$ apply in particular when $j=1$, in which case they may or may not duplicate the methods of Vaughan $[13,14]$.

5. Iterative schemes based on the Hardy-Littlewood method.

We next investigate estimates arising from iterative schemes of the shape

$$
\begin{aligned}
& F_{0}^{2} f_{0}^{2 s-2}
\end{aligned}
$$

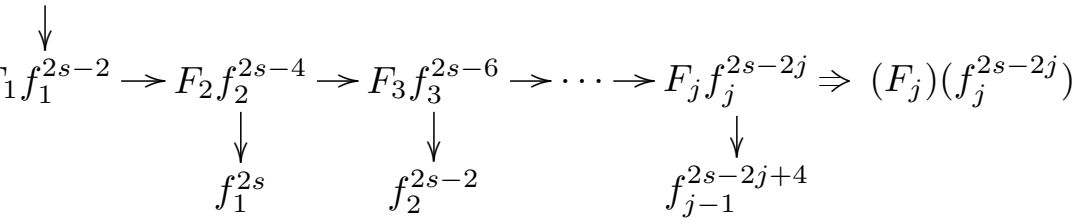

and

$\left(\mathrm{M}_{2}\right)$

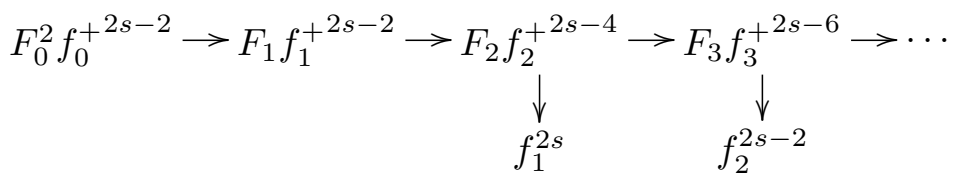

$$
\begin{aligned}
& \cdots \rightarrow F_{j-1} f_{j-1}^{+2 s-2 j+2} \rightarrow F_{j} g_{j}^{2} f_{j}^{2 s-2 j-2} \Rightarrow\left(F_{j}\right)\left(g_{j}^{2} f_{j}^{2 s-2 j-2}\right) \\
& \underset{f_{j-2}^{2 s-2 j+6}}{\downarrow} \underset{f_{j-1}^{2 s-2 j+4}}{\downarrow}
\end{aligned}
$$


A perusal of the arguments of [18] should convince the reader that the derivation of bounds close to optimal via the Hardy-Littlewood method in such schemes is a matter of considerable complexity. We therefore strive for simplicity, sacrificing a little on performance.

In the first iterative scheme above, we estimate the mean value occurring in the final step of the iterative procedure by means of Lemma 13.1 of [18], which we record in a slightly more general form. We first require some notation.

Definition 5.1. Suppose that $k \geq 4$ and $1 \leq j \leq k-3$.

(i) Let $\mathfrak{M}_{j}$ denote the union of the intervals

$$
\mathfrak{M}_{j}(q, a)=\left\{\alpha \in[0,1):|q \alpha-a| \leq P Q_{j}^{-k} R^{k(j-k)}\right\},
$$

with $0 \leq a \leq q \leq P$ and $(a, q)=1$. Also, let $\mathfrak{m}_{j}=[0,1) \backslash \mathfrak{M}_{j}$.

(ii) Define $\varpi_{j}$ to be 0 when $j=k-3$, and to be 1 when $1 \leq j \leq k-4$. Also, write $w_{j}=2^{1+j-k}$.

(iii) Let $\mathfrak{N}_{j}$ denote the union of the intervals

$$
\mathfrak{N}_{j}(q, a)=\left\{\alpha \in[0,1):|q \alpha-a| \leq\left(P M_{1}^{\varpi_{j}}\right)^{w_{j}(k-j)} Q_{j}^{-k}\right\},
$$

with $0 \leq a \leq q \leq\left(P M_{1}^{\varpi_{j}}\right)^{w_{j}(k-j)}$ and $(a, q)=1$.

We note that the $\mathfrak{M}_{j}(q, a)$ comprising $\mathfrak{M}_{j}$ are disjoint, and likewise also the $\mathfrak{N}_{j}(q, a)$ comprising $\mathfrak{N}_{j}$.

Lemma 5.2. Suppose that $k \geq 4$ and $1 \leq j \leq k-3$. Let $u$ be a positive integer, and define

$$
t=\left[\left(\frac{k-j+1}{k-j}\right) u+1\right], \quad \theta=t-\left(\frac{k-j+1}{k-j}\right) u .
$$

Suppose that $\Delta_{t-1}$ and $\Delta_{t}$ are admissible exponents, and write

$$
\mu_{u}=\frac{k-j}{k-j+1}\left(\theta \Delta_{t-1}+(1-\theta) \Delta_{t}\right) .
$$

Then whenever $\Delta_{u}$ is an admissible exponent,

$$
\int_{0}^{1}\left|F_{j}(\alpha) f_{j}(\alpha)^{2 u}\right| d \alpha \ll P^{1+\varepsilon} \widetilde{H}_{j} \widetilde{M}_{j} Q_{j}^{2 u-k}\left(\left(P M_{1}^{\varpi_{j}}\right)^{-w_{j}} Q_{j}^{\Delta_{u}}+Q_{j}^{\mu_{u}}\right) .
$$

Pr o of. When $1 \leq j \leq k-4$, the stated conclusion is provided by Lemma 13.1 of [18]. When $j=k-3$, meanwhile, the conclusion follows from the argument of the proof of the latter lemma, noting that by a Weyl differencing argument paralleling those of Lemmata 6.1 and 12.1 of [18], it follows from Lemma 4.1 of [18] together with a trivial estimate that

$$
\sup _{\alpha \in \mathfrak{m}_{k-3}}\left|F_{k-3}(\alpha)\right| \ll P^{1-w_{k-3}+\varepsilon} \widetilde{H}_{k-3} \widetilde{M}_{k-3} .
$$


We next consider the second of the iterative schemes above, but in order to make further progress we require some additional notation. When $k \geq 2$, we write

$$
S_{k}(q, a)=\sum_{r=1}^{q} e\left(a r^{k} / q\right)
$$

and define also the multiplicative function $w_{k}(q)$ by taking

$$
w_{k}\left(p^{u k+v}\right)= \begin{cases}k p^{-u-1 / 2} & \text { when } u \geq 0 \text { and } v=1, \\ p^{-u-1} & \text { when } u \geq 0 \text { and } 2 \leq v \leq k .\end{cases}
$$

Note that there is some possibility of confusion between the function $w_{k}(q)$ and the exponent $w_{j}$, but that a perusal of the context should easily dispel any ambiguity. Then according to Lemma 3 of Vaughan [12], whenever $a \in \mathbb{Z}$ and $q \in \mathbb{N}$ satisfy $(a, q)=1$, one has

$$
q^{-1 / 2} \leq w_{k}(q) \ll q^{-1 / k}
$$

and

$$
q^{-1} S_{k}(q, a) \ll w_{k}(q) .
$$

We require the estimate contained in the following lemma.

Lemma 5.3. Suppose that $k \geq 4$ and $1 \leq j \leq k-3$. Then whenever $\alpha \in \mathfrak{N}_{j}(q, a) \subseteq \mathfrak{N}_{j}$, one has

$$
g_{j}(\alpha) \ll Q_{j}^{1+\varepsilon} w_{k}(q)\left(1+Q_{j}^{k}|\alpha-a / q|\right)^{-1}+P^{\varepsilon}\left(P M_{1}^{\varpi_{j}}\right)^{w_{j}(k-j) / 2} .
$$

If in addition one has $Q_{j} \geq\left(P M_{1}^{\varpi_{j}}\right)^{w_{j}(k-j)}$, then

$$
g_{j}(\alpha) \ll Q_{j}^{1+\varepsilon} w_{k}(q)\left(1+Q_{j}^{k}|\alpha-a / q|\right)^{-1} .
$$

Proof. On making use of the refinements embodied in Theorem 4.1 of Vaughan [15], we deduce that whenever $\alpha \in \mathfrak{N}_{j}(q, a) \subseteq \mathfrak{N}_{j}$, one has

$$
g_{j}(\alpha)-q^{-1} S_{k}(q, a) v_{j}^{+}(\beta) \ll q^{1 / 2+\varepsilon}\left(1+Q_{j}^{k}|\alpha-a / q|\right)^{A / 2},
$$

where $A$ is 0 or 1 according to whether or not $Q_{j} \geq\left(P M_{1}^{\varpi_{j}}\right)^{w_{j}(k-j)}$, and

$$
v_{j}^{+}(\beta)=\int_{Q_{j} R^{-j} / 2}^{Q_{j}} e\left(\beta \gamma^{k}\right) d \gamma .
$$

By partial integration, one readily deduces that

$$
v_{j}^{+}(\beta) \ll \min \left\{Q_{j},\left(Q_{j} R^{-j}\right)^{1-k}|\beta|\right\} \ll \frac{Q_{j} R^{j(k-1)}}{1+Q_{j}^{k}|\beta|} .
$$


On recalling (5.2), therefore, we deduce that for $\alpha \in \mathfrak{N}_{j}(q, a) \subseteq \mathfrak{N}_{j}$, one has the first conclusion of the lemma.

If $Q_{j} \geq\left(P M_{1}^{\varpi_{j}}\right)^{w_{j}(k-j)}$, the term on the right hand side of (5.3) becomes $q^{1 / 2+\varepsilon}$, and so it follows from (2.1) and (5.1) that for $\alpha \in \mathfrak{N}_{j}(q, a) \subseteq \mathfrak{N}_{j}$, the upper bound for $g_{j}(\alpha)$ concluding the lemma majorises the right hand side of (5.3). The second conclusion of the lemma is now immediate.

We must also estimate $F_{j}(\alpha)$ for $\alpha \in \mathfrak{M}_{j}$ in order to prosecute the estimation required for the use of the second iterative scheme. In this context, we write

$$
\tau_{j}(q, a, \mathbf{h}, \mathbf{m})=\left|\sum_{r=1}^{q} e\left(\frac{a}{q} \Psi_{j}(r, \mathbf{h}, \mathbf{m})\right)\right|,
$$

and then define $F_{j}^{*}(\alpha)$ to be zero whenever $\alpha \in \mathfrak{m}_{j}$, and

$$
F_{j}^{*}(\alpha)=\sum_{\mathbf{m}} \sum_{\mathbf{h}} \frac{P q^{-1} \tau_{j}(q, a, \mathbf{h}, \mathbf{m})}{\left(1+|\alpha-a / q| h_{1} \ldots h_{j} P^{k-j}\right)^{1 /(k-j)}},
$$

when $\alpha \in \mathfrak{M}_{j}(q, a) \subseteq \mathfrak{M}_{j}$. Here, the summation is over $\mathbf{m}$ and $\mathbf{h}$ satisfying (2.1). Finally, we define $g_{j}^{*}(\alpha)$ to be zero for $\alpha \in \mathfrak{n}_{j}$, and

$$
g_{j}^{*}(\alpha)=Q_{j}^{1+\varepsilon} w_{k}(q)\left(1+Q_{j}^{k}|\alpha-a / q|\right)^{-1},
$$

when $\alpha \in \mathfrak{N}_{j}(q, a) \subseteq \mathfrak{N}_{j}$. We observe that this definition of $g_{j}^{*}(\alpha)$ differs from that provided in Section 2 of Vaughan and Wooley [16], but not in a manner damaging to our subsequent argument.

We now describe an auxiliary lemma which may be of interest beyond this work. Our treatment here is motivated by the proof of Lemma 3.1 of Brüdern and Wooley [4].

Lemma 5.4. Suppose that $k \geq 4$. Let $Q$ be a real number with $1 \leq Q \leq P$. Let $\mathfrak{M}$ denote the union of the intervals

$$
\mathfrak{M}(q, a)=\left\{\alpha \in[0,1):|q \alpha-a| \leq Q P^{-k}\right\},
$$

with $0 \leq a \leq q \leq Q$ and $(a, q)=1$. Let $\delta$ be a real number with $\delta>1$, and define the function $\Upsilon(\alpha)$ for $\alpha \in \mathfrak{M}$ by taking

$$
\Upsilon(\alpha)=w_{k}(q)^{2}\left(1+P^{k}|\alpha-a / q|\right)^{-\delta}
$$

when $\alpha \in \mathfrak{M}(q, a) \subseteq \mathfrak{M}$. Also, write $t=[k / 2]$. Then for any subset $\mathcal{A}$ of $[1, P] \cap \mathbb{Z}$, one has for each $\varepsilon>0$ the estimate

$$
\int_{\mathfrak{M}} \Upsilon(\alpha)\left|\sum_{x \in \mathcal{A}} e\left(\alpha x^{k}\right)\right|^{2 t} d \alpha \ll Q^{\varepsilon} P^{2 t-k}
$$


Proof. We begin by observing that

$$
\begin{aligned}
& \int_{\mathfrak{M}} \Upsilon(\alpha)\left|\sum_{x \in \mathcal{A}} e\left(\alpha x^{k}\right)\right|^{2 t} d \alpha \\
\leq & \sum_{1 \leq q \leq Q} w_{k}(q)^{2} \int_{-Q / P^{k}}^{Q / P^{k}}\left(1+P^{k}|\beta|\right)^{-\delta} \sum_{a=1}^{q}\left|\sum_{x \in \mathcal{A}} e\left(x^{k}(\beta+a / q)\right)\right|^{2 t} d \beta .
\end{aligned}
$$

By orthogonality,

$$
\sum_{a=1}^{q}\left|\sum_{x \in \mathcal{A}} e\left(x^{k}(\beta+a / q)\right)\right|^{2 t}=q \sum_{\substack{\mathbf{x} \in \mathcal{A}^{2 t} \\ q \mid \psi(\mathbf{x})}} e(\beta \psi(\mathbf{x})),
$$

where we write

$$
\psi(\mathbf{x})=\sum_{i=1}^{t}\left(x_{2 i-1}^{k}-x_{2 i}^{k}\right)
$$

But plainly,

$$
\sum_{\substack{\mathbf{x} \in \mathcal{A}^{2 t} \\ q \mid \psi(\mathbf{x})}} e(\beta \psi(\mathbf{x})) \leq \sum_{\substack{1 \leq x_{1}, \ldots, x_{2 t} \leq P \\ q \mid \psi(\mathbf{x})}} 1 \leq\left(P q^{-1}+1\right)^{2 t} \varrho(q),
$$

where $\varrho(q)$ denotes the number of solutions of the congruence

$$
\sum_{i=1}^{t}\left(x_{2 i-1}^{k}-x_{2 i}^{k}\right) \equiv 0(\bmod q)
$$

with $1 \leq x_{i} \leq q(1 \leq i \leq 2 t)$. By orthogonality, moreover, it follows from (5.6) that

$$
q \varrho(q)=\sum_{a=1}^{q}\left|S_{k}(q, a)\right|^{2 t}=\sum_{a=1}^{q}(q, a)^{2 t}\left|S_{k}\left(\frac{q}{(q, a)}, \frac{a}{(q, a)}\right)\right|^{2 t},
$$

whence by (5.2),

$$
q \varrho(q) \ll q^{2 t} \sum_{a=1}^{q} w_{k}(q /(q, a))^{2 t}=q^{2 t} \sum_{r \mid q} r w_{k}(r)^{2 t} .
$$

Consequently, on inserting this estimate into (5.7) and substituting into (5.4) and (5.5), we deduce that

$$
\int_{\mathfrak{M}} \Upsilon(\alpha)\left|\sum_{x \in \mathcal{A}} e\left(\alpha x^{k}\right)\right|^{2 t} d \alpha \ll P^{2 t-k} \sum_{1 \leq q \leq Q} w_{k}(q)^{2} \sigma(q),
$$


where

$$
\sigma(q)=\sum_{r \mid q} r w_{k}(r)^{2 t}
$$

The function $w_{k}(r)$ is multiplicative with respect to $r$, and thus $\sigma(q)$ is likewise a multiplicative function of $q$. Further, it follows from (5.9) that for each prime $p$ and natural number $h$, one has

$$
\sigma\left(p^{h}\right)=\sum_{l=0}^{h} p^{l} w_{k}\left(p^{l}\right)^{2 t}
$$

whence by the definition of $w_{k}(q)$,

$$
\sigma\left(p^{h}\right)=1+\sum_{u k+1 \leq h} p^{u k+1}\left(k p^{-u-1 / 2}\right)^{2 t}+\sum_{v=2}^{k} \sum_{u k+v \leq h} p^{u(k-2 t)+v-2 t} .
$$

Thus, on recalling that $t=[k / 2]$, we deduce that

$$
\sigma(p) \leq 1+k^{2 t} p^{-1}
$$

and for $h \geq 2$ we obtain

$$
\begin{aligned}
\sigma\left(p^{h}\right) & \ll_{k} p^{\frac{h-1}{k}(k-2 t)+1-t}+\sum_{v=2}^{k} p^{\frac{h-v}{k}(k-2 t)+v-2 t} \\
& \ll_{k} p^{\frac{h-1}{k}(k-2 t)+1-t}+p^{\frac{h}{k}(k-2 t)} \ll p^{h / k} .
\end{aligned}
$$

We therefore arrive at the estimates

$$
\begin{aligned}
w_{k}(p)^{2} \sigma(p) & \ll_{k} p^{-1}, \\
w_{k}\left(p^{u k+1}\right)^{2} \sigma\left(p^{u k+1}\right) & \ll_{k} p^{-u-1+1 / k} \quad(u \geq 1), \\
w_{k}\left(p^{u k+v}\right)^{2} \sigma\left(p^{u k+v}\right) & \ll_{k} p^{-u-1} \quad(u \geq 0 \text { and } 2 \leq v \leq k) .
\end{aligned}
$$

The multiplicative properties of $\sigma(q)$ and $w_{k}(q)$ consequently assure us that for a suitable constant $A$ depending at most on $k$,

$$
\begin{aligned}
\sum_{1 \leq q \leq Q} w_{k}(q)^{2} \sigma(q) & \leq \prod_{p \leq Q}\left(1+\sum_{h=1}^{\infty} w_{k}\left(p^{h}\right)^{2} \sigma\left(p^{h}\right)\right) \\
& \leq \prod_{p \leq Q}\left(1+A p^{-1}\right) \ll Q^{\varepsilon}
\end{aligned}
$$

The conclusion of the lemma now follows immediately from (5.8).

We record an immediate corollary of Lemma 5.4 in the form of the following lemma.

Lemma 5.5. Suppose that $k \geq 4,1 \leq j \leq k-3$ and $u \geq[k / 2]$. Suppose also that

$$
Q_{j} \geq\left(P M^{\varpi_{j}}\right)^{w_{j}(k-j)} .
$$


Then whenever $\Delta_{u+1}$ is an admissible exponent,

where

$$
\int_{0}^{1}\left|F_{j}(\alpha) g_{j}(\alpha)^{2} f_{j}(\alpha)^{2 u}\right| d \alpha \ll P^{1+\varepsilon} \widetilde{M}_{j} \widetilde{H}_{j} Q_{j}^{2 u+2-k} \mathcal{M},
$$

$$
\mathcal{M}=\left(P M_{1}^{\varpi_{j}}\right)^{-w_{j}} Q_{j}^{\Delta_{u+1}}+1 .
$$

Proof. Following the argument of the proof of Lemma 3.1 of [16] (see, in particular, equations (3.1), (3.7) and (3.8) of that paper), we obtain

$$
\begin{aligned}
& \int_{0}^{1}\left|F_{j}(\alpha) g_{j}(\alpha)^{2} f_{j}(\alpha)^{2 u}\right| d \alpha \\
& \ll P^{1+\varepsilon} \widetilde{H}_{j} \widetilde{M}_{j} Q_{j}^{2 u+2-k}\left(P M_{1}^{\varpi_{j}}\right)^{-w_{j}} Q_{j}^{\Delta_{u+1}}+I
\end{aligned}
$$

where

$$
I=\int_{\mathfrak{N}_{j}} F_{j}^{*}(\alpha)\left|g_{j}(\alpha)^{2} f_{j}(\alpha)^{2 u}\right| d \alpha .
$$

We note here that our choice of $\varpi_{j}$ when $j=k-3$ ensures that the above conclusion remains valid also when $j=k-3$. Since by hypothesis we have

$$
\left(P M_{1}^{\varpi_{j}}\right)^{w_{j}(k-j)} \leq Q_{j},
$$

it follows from Lemma 5.3 that for $\alpha \in \mathfrak{N}_{j}(q, a) \subseteq \mathfrak{N}_{j}$, one has

$$
g_{j}(\alpha) \ll Q_{j}^{1+\varepsilon} w_{k}(q)\left(1+Q_{j}^{k}|\alpha-a / q|\right)^{-1} .
$$

Then on making use of a trivial estimate for $F_{j}^{*}(\alpha)$, we deduce from (5.11) that

$$
I \ll P \widetilde{M}_{j} \widetilde{H}_{j} Q_{j}^{2+\varepsilon} \int_{\mathfrak{N}_{j}} \Upsilon_{j}(\alpha)\left|f_{j}(\alpha)\right|^{2 u} d \alpha,
$$

where $\Upsilon_{j}(\alpha)$ is the function defined for $\alpha \in \mathfrak{N}_{j}$ by taking

$$
\Upsilon_{j}(\alpha)=w_{k}(q)^{2}\left(1+Q_{j}^{k}|\alpha-a / q|\right)^{-2},
$$

when $\alpha \in \mathfrak{N}_{j}(q, a) \subseteq \mathfrak{N}_{j}$. But the hypotheses of the statement of Lemma 5.4 are satisfied for $\Upsilon_{j}(\alpha)$ on $\mathfrak{N}_{j}$, whence for $u \geq[k / 2]$ we find that

$$
\int_{\mathfrak{N}_{j}} \Upsilon_{j}(\alpha)\left|f_{j}(\alpha)\right|^{2 u} d \alpha \ll Q_{j}^{2 u-k+\varepsilon} .
$$

Thus we conclude from (5.12) and (5.13) that

$$
I \ll P^{1+\varepsilon} \widetilde{M}_{j} \widetilde{H}_{j} Q_{j}^{2 u+2-k},
$$

and hence the desired conclusion is immediate from (5.10).

We supplement Lemma 5.5 with a variant of Lemmata 3.1 and 3.2 of [16] which is of interest when

$$
\left(P M_{1}^{\varpi_{j}}\right)^{w_{j}(k-j) / 2}<Q_{j}<\left(P M_{1}^{\varpi_{j}}\right)^{w_{j}(k-j)} .
$$


Lemma 5.6. Suppose that $k \geq 4$ and $1 \leq j \leq k-3$, and suppose also that the condition (5.14) holds. Let $u$ be a positive integer, and define $t$ and $\theta$ as in the statement of Lemma 5.2. Suppose further that $\Delta_{t-1}$ and $\Delta_{t}$ are admissible exponents, and define $\mu_{u}$ also as in the statement of Lemma 5.2. Define next

$$
\gamma=1-\frac{2}{k}-\frac{1}{k-j+1}, \quad v=u-\frac{4}{k}, \quad w=\left[\gamma^{-1} v+1\right]
$$

and

$$
\theta^{\prime}=w-\gamma^{-1} v
$$

Suppose that $\Delta_{w-1}$ and $\Delta_{w}$ are admissible exponents, and write

$$
\varrho_{u}=\gamma\left(\theta^{\prime} \Delta_{w-1}+\left(1-\theta^{\prime}\right) \Delta_{w}\right) .
$$

Then whenever $\Delta_{u+1}$ is an admissible exponent,

$$
\int_{0}^{1}\left|F_{j}(\alpha) g_{j}(\alpha)^{2} f_{j}(\alpha)^{2 u}\right| d \alpha \ll P^{1+\varepsilon} \widetilde{H}_{j} \widetilde{M}_{j} Q_{j}^{2 u+2-k} \mathcal{M},
$$

where

$$
\mathcal{M}=\left(P M_{1}^{\varpi_{j}}\right)^{-w_{j}} Q_{j}^{\Delta_{u+1}}+\left(P M_{1}^{\varpi_{j}}\right)^{(k-j) w_{j}} Q_{j}^{\mu_{u}-2}+Q_{j}^{\varrho_{u}} .
$$

Proof. Following the argument of the proof of Lemma 3.1 of [16] (see, in particular, equations (3.1), (3.7)-(3.9) and (3.13)), we find that

$$
\int_{0}^{1}\left|F_{j}(\alpha) g_{j}(\alpha)^{2} f_{j}(\alpha)^{2 u}\right| d \alpha \ll P^{1+\varepsilon} \widetilde{H}_{j} \widetilde{M}_{j} Q_{j}^{2 u+2-k} \mathcal{M}^{\prime}+J_{1},
$$

where

$$
\mathcal{M}^{\prime}=\left(P M_{1}^{\varpi_{j}}\right)^{-w_{j}} Q_{j}^{\Delta_{u+1}}+\left(P M_{1}^{\varpi_{j}}\right)^{(k-j) w_{j}} Q_{j}^{\mu_{u}-2}
$$

and

$$
J_{1}=\int_{\mathfrak{N}_{j}} F_{j}^{*}(\alpha) g_{j}^{*}(\alpha)^{2}\left|f_{j}(\alpha)\right|^{2 u} d \alpha .
$$

Here we note that our definition of $g_{j}^{*}(\alpha)$ differs from that of [16], the substitution of the present definition being permitted through the use of Lemma 5.3.

We estimate the mean value $J_{1}$ via Hölder's inequality, deducing from (5.17) that

$$
J_{1} \leq J_{2}^{1 /(k-j+1)} J_{3}^{2 / k}\left(J_{4}^{\theta^{\prime}} J_{5}^{1-\theta^{\prime}}\right)^{\gamma},
$$


where

$$
\begin{aligned}
J_{2} & =\int_{\mathfrak{N}_{j}} F_{j}^{*}(\alpha)^{k-j+1} d \alpha, & J_{3} & =\int_{\mathfrak{N}_{j}} g_{j}^{*}(\alpha)^{k}\left|f_{j}(\alpha)\right|^{4} d \alpha, \\
J_{4} & =\int_{0}^{1}\left|f_{j}(\alpha)\right|^{2 w-2} d \alpha, & J_{5} & =\int_{0}^{1}\left|f_{j}(\alpha)\right|^{2 w} d \alpha .
\end{aligned}
$$

But since $\Delta_{w-1}$ and $\Delta_{w}$ are admissible exponents, one has

$$
J_{4} \ll Q_{j}^{2 w-2-k+\Delta_{w-1}+\varepsilon} \text { and } J_{5} \ll Q_{j}^{2 w-k+\Delta_{w}+\varepsilon} .
$$

Also, it is a consequence of Lemma 4.10 of [18] that

$$
J_{2} \ll P^{\varepsilon}\left(P \widetilde{H}_{j} \widetilde{M}_{j}\right)^{k-j+1} Q_{j}^{-k} .
$$

In order to estimate $J_{3}$ we first note that by (5.1), whenever $\alpha \in \mathfrak{N}_{j}(q, a)$ $\subseteq \mathfrak{N}_{j}$, one has

$$
g_{j}^{*}(\alpha)^{k}=w_{k}(q)^{k} Q_{j}^{k+\varepsilon}\left(1+Q_{j}^{k}|\alpha-a / q|\right)^{-k} \ll Q_{j}^{k+\varepsilon}\left(q+Q_{j}^{k}|q \alpha-a|\right)^{-1} .
$$

When $h$ is an integer, write $\psi_{h}$ for the number of solutions of the equation

$$
x_{1}^{k}+x_{2}^{k}-x_{3}^{k}-x_{4}^{k}=h,
$$

with $x_{i} \in \mathcal{A}\left(Q_{j}, R\right)(1 \leq i \leq 4)$. Then plainly,

$$
\left|f_{j}(\alpha)\right|^{4}=\sum_{|h| \leq 2 Q_{j}^{k}} \psi_{h} e(\alpha h),
$$

and thus it is a consequence of Lemma 2 of Brüdern [3] that

$$
\int_{\mathfrak{N}_{j}} g_{j}^{*}(\alpha)^{k}\left|f_{j}(\alpha)\right|^{4} d \alpha \ll Q_{j}^{\varepsilon}\left(\left(P M_{1}^{\varpi_{j}}\right)^{w_{j}(k-j)} \psi_{0}+\sum_{h \neq 0}\left|\psi_{h}\right|\right) .
$$

But by Hua's Lemma (see, for example, Lemma 2.5 of Vaughan [15]), together with an elementary counting argument,

$$
\psi_{0} \ll Q_{j}^{2+\varepsilon} \text { and } \quad \sum_{h \in \mathbb{Z}}\left|\psi_{h}\right| \ll Q_{j}^{4} .
$$

Then on recalling that our hypotheses imply that

$$
Q_{j}>\left(P M_{1}^{\varpi_{j}}\right)^{w_{j}(k-j) / 2},
$$

we conclude from (5.21) that

$$
J_{3}=\int_{\mathfrak{N}_{j}} g_{j}^{*}(\alpha)^{k}\left|f_{j}(\alpha)\right|^{4} d \alpha \ll Q_{j}^{4+\varepsilon} .
$$

On combining (5.18)-(5.20) and (5.22), we arrive at the estimate

$$
J_{1} \ll P^{1+\varepsilon} \widetilde{H}_{j} \widetilde{M}_{j} Q_{j}^{2 u+2-k+\varrho_{u}},
$$

and so the conclusion of the lemma is immediate from (5.15) and (5.16). 
Our next task is to assemble the estimates described above into a tool sufficiently simple to apply that it is viable to employ computationally. Our aim is to establish either that

$$
\begin{aligned}
\int_{0}^{1}\left|F_{j}(\alpha) f_{j}(\alpha)^{2 u+2}\right| d \alpha & \\
& \ll P^{1+\varepsilon} \widetilde{H}_{j} \widetilde{M}_{j} Q_{j}^{2 u+2-k}\left(\left(P M_{1}^{\varpi_{j}}\right)^{-w_{j}} Q_{j}^{\Delta_{u+1}}+1\right),
\end{aligned}
$$

or else that

$$
\begin{aligned}
& \int_{0}^{1}\left|F_{j}(\alpha) g_{j}(\alpha)^{2} f_{j}(\alpha)^{2 u}\right| d \alpha \\
& \ll P^{1+\varepsilon} \widetilde{H}_{j} \widetilde{M}_{j} Q_{j}^{2 u+2-k}\left(\left(P M_{1}^{\varpi_{j}}\right)^{-w_{j}} Q_{j}^{\Delta_{u+1}}+1\right) .
\end{aligned}
$$

Thus we seek to show that the mean values on the left hand sides of (5.23) and (5.24) are bounded above by the estimate for the minor arc contribution stemming from our methods, together with the "expected" major arc contribution. In order to ease our discussion, we list a number of conditions concerning the quantities $\mu_{u}, \varrho_{u}$ and $\Delta_{u}$ defined in the statements of Lemmata 5.2, 5.5 and 5.6.

$$
\begin{array}{cc}
\left(\mathrm{A}_{1}\right) & w_{j}\left(1+\varpi_{j} \phi_{j}\right) \leq\left(\Delta_{u+1}-\mu_{u+1}\right)\left(1-\phi_{1}-\ldots-\phi_{j}\right), \\
\left(\mathrm{A}_{2}\right) & \mu_{u+1} \leq 0, \\
(\mathrm{~B}) & u \geq[k / 2] \quad \text { and } \quad w_{j}(k-j)\left(1+\varpi_{j} \phi_{1}\right) \leq 1-\phi_{1}-\ldots-\phi_{j}, \\
\left(\mathrm{C}_{1}\right) & \frac{1}{2} w_{j}(k-j)\left(1+\varpi_{j} \phi_{1}\right) \leq 1-\phi_{1}-\ldots-\phi_{j} \leq w_{j}(k-j)\left(1+\varpi_{j} \phi_{1}\right), \\
\left(\mathrm{C}_{2}\right) & w_{j}\left(1+\varpi_{j} \phi_{1}\right) \leq\left(\Delta_{u+1}-\varrho_{u}\right)\left(1-\phi_{1}-\ldots-\phi_{j}\right), \\
\left(\mathrm{C}_{3}\right) & w_{j}(k-j+1)\left(1+\varpi_{j} \phi_{j}\right) \leq\left(2+\Delta_{u+1}-\mu_{u}\right)\left(1-\phi_{1}-\ldots-\phi_{j}\right), \\
\left(\mathrm{C}_{4}\right) & w_{j}(k-j)\left(1+\varpi_{j} \phi_{1}\right) \leq\left(2-\mu_{u}\right)\left(1-\phi_{1}-\ldots-\phi_{j}\right), \\
\left(\mathrm{C}_{5}\right) & \varrho_{u} \leq 0, \\
\left(\mathrm{D}_{1}\right) & \Delta_{u+1}\left(1-\phi_{1}-\ldots-\phi_{j}\right)>w_{j}\left(1+\varpi_{j} \phi_{1}\right), \\
\left(\mathrm{D}_{2}\right) & \Delta_{u+1}\left(1-\phi_{1}-\ldots-\phi_{j}\right) \leq w_{j}\left(1+\varpi_{j} \phi_{1}\right) .
\end{array}
$$

We now summarise the conclusions of Lemmata 5.2, 5.5 and 5.6.

Lemma 5.7. Let $k \geq 4$ and $1 \leq j \leq k-3$.

(I) Suppose that condition $\left(\mathrm{D}_{1}\right)$ holds, and further that one of the conditions $(\mathrm{B})$, or each of $\left(\mathrm{C}_{1}\right),\left(\mathrm{C}_{2}\right),\left(\mathrm{C}_{3}\right)$ holds. Then

$$
\int_{0}^{1}\left|F_{j}(\alpha) g_{j}(\alpha)^{2} f_{j}(\alpha)^{2 u}\right| d \alpha \ll P^{1+\varepsilon} \widetilde{H}_{j} \widetilde{M}_{j} Q_{j}^{2 u+2-k}\left(P M_{1}^{\varpi_{j}}\right)^{-w_{j}} Q_{j}^{\Delta_{u+1}} .
$$


$\left(\mathrm{I}^{\prime}\right)$ Suppose that the condition $\left(\mathrm{A}_{1}\right)$ holds. Then

$$
\int_{0}^{1}\left|F_{j}(\alpha) f_{j}(\alpha)^{2 u+2}\right| d \alpha \ll P^{1+\varepsilon} \widetilde{H}_{j} \widetilde{M}_{j} Q_{j}^{2 u+2-k}\left(P M_{1}^{\varpi_{j}}\right)^{-w_{j}} Q_{j}^{\Delta_{u+1}} .
$$

(II) Suppose that condition $\left(\mathrm{D}_{2}\right)$ holds, and further that one of the conditions $(\mathrm{B})$, or each of $\left(\mathrm{C}_{1}\right),\left(\mathrm{C}_{4}\right)$ and $\left(\mathrm{C}_{5}\right)$ holds. Then

$$
\int_{0}^{1}\left|F_{j}(\alpha) g_{j}(\alpha)^{2} f_{j}(\alpha)^{2 u}\right| d \alpha \ll P^{1+\varepsilon} \widetilde{H}_{j} \widetilde{M}_{j} Q_{j}^{2 u+2-k} .
$$

$(\mathrm{II})$ Suppose that condition $\left(\mathrm{D}_{2}\right)$ holds, and further that condition $\left(\mathrm{A}_{2}\right)$ holds. Then

$$
\int_{0}^{1}\left|F_{j}(\alpha) f_{j}(\alpha)^{2 u+2}\right| d \alpha \ll P^{1+\varepsilon} \widetilde{H}_{j} \widetilde{M}_{j} Q_{j}^{2 u+2-k} .
$$

Proof. The assertions of each case of the lemma are immediate, save that in part $\left(\mathrm{I}^{\prime}\right)$ we have made the observation that the validity of condition $\left(\mathrm{A}_{1}\right)$ automatically implies that of $\left(\mathrm{D}_{1}\right)$.

Since for each $i$ one has $0 \leq \phi_{i} \leq 1 / k$, one deduces readily that the condition $(\mathrm{B})$ is satisfied automatically whenever

$$
u \geq[k / 2] \quad \text { and } \quad 2^{1+j-k}\left(k+\varpi_{j}\right) \leq 1 .
$$

In particular, therefore, the condition (B) is satisfied when $u \geq[k / 2]$ and

$$
j \leq k-4 \text { and } k \leq 7
$$

or

$$
j \leq k-5 \quad \text { and } \quad k \leq 15 \text {, }
$$

or

$$
j \leq k-6 \text { and } k \leq 31 .
$$

We finish this section by indicating how to obtain new permissible exponents via the iterative schemes $\left(\mathrm{M}_{1}\right)$ and $\left(\mathrm{M}_{2}\right)$. We suppose in what follows that $\lambda_{r}(r \in \mathbb{N})$ are known permissible exponents, and we seek a new permissible exponent $\lambda_{s}^{\prime}$.

(i) Process $M_{j}^{s}$. Consider first the iterative scheme $\left(\mathrm{M}_{2}\right)$ above. Suppose that the conditions of Lemma 5.7(I) hold with $u=s-j-1$. Then following the argument of Section 2 of [18] (see also Sections 11, 13), we find from Lemma 5.7(I) that $\lambda_{s}^{\prime}$ and $\phi$ are determined by the relations

$$
P \widetilde{H}_{j-1} \widetilde{M}_{j} Q_{j}^{\lambda_{s-j}} \approx P \widetilde{H}_{j} \widetilde{M}_{j} Q_{j}^{\lambda_{s-j}}\left(P M_{1}^{\varpi_{j}}\right)^{-w_{j}},
$$


and the equations (4.2)-(4.4). Define $\mathcal{E}_{i}$ as in (4.5) for $1 \leq i<j$, and define $\kappa_{i}$ as in (4.6) for $2 \leq i \leq j$. Also, define $\alpha_{i}, \beta_{i}, \gamma_{i}, \delta_{i}$ for $1 \leq i \leq j$ by

$$
\alpha_{j}=k^{-1}, \quad \beta_{j}=0, \quad \gamma_{j}=1-w_{j}, \quad \delta_{j}=w_{j} \varpi_{j},
$$

and for $i=j-1, \ldots, 2$ successively by

$$
\begin{aligned}
\delta_{i} & =\mathcal{E}_{i}+\kappa_{i+1} \alpha_{i+1} \delta_{i+1}, \\
\gamma_{i} & =1+\mathcal{E}_{i}+\kappa_{i+1} \alpha_{i+1} \gamma_{i+1}, \\
\beta_{i} & =\mathcal{E}_{i}+\kappa_{i+1} \alpha_{i+1} \beta_{i+1}, \\
\alpha_{i} & =\left(2 k+\beta_{i}\right)^{-1},
\end{aligned}
$$

and finally,

$$
\begin{aligned}
\delta_{1} & =\mathcal{E}_{1}+\kappa_{2} \alpha_{2} \delta_{2}, \\
\gamma_{1} & =1+\mathcal{E}_{1}+\kappa_{2} \alpha_{2} \gamma_{2}, \\
\beta_{1} & =0, \\
\alpha_{1} & =\left(2 k+\delta_{1}\right)^{-1} .
\end{aligned}
$$

Then we find that $\phi$ and $\lambda_{s}^{\prime}$ satisfy

$$
\begin{gathered}
\phi_{i}=\alpha_{i}\left(\gamma_{i}-\beta_{i}\left(\phi_{2}+\ldots+\phi_{i-1}\right)-\delta_{i} \phi_{1}\right) \quad(2 \leq i \leq j), \\
\phi_{1}=\alpha_{1} \gamma_{1}
\end{gathered}
$$

and

$$
\lambda_{s}^{\prime}=\lambda_{s-1}\left(1-\phi_{1}\right)+1+(2 s-2) \phi_{1} .
$$

Thus, in a manner similar to that alluded to in Section 4 , we are able to establish new permissible exponents by iterating this and allied procedures.

Suppose next that the conditions of Lemma 5.7(II) hold with $u=s-j-1$. Then again following the argument of Section 2 of [18], we now find from Lemma 5.7(II) that $\lambda_{s}^{\prime}$ and $\phi$ are determined by the relations

$$
P \widetilde{H}_{j-1} \widetilde{M}_{j} Q_{j}^{\lambda_{s-j}} \approx P \widetilde{H}_{j} \widetilde{M}_{j} Q_{j}^{2 s-2 j-k},
$$

and the equations (4.2)-(4.4). Define $\mathcal{E}_{i}$ as in (4.5) for $1 \leq i<j$, and define $\kappa_{i}$ as in (4.6) for $2 \leq i \leq j$. Also, define $\alpha_{i}, \beta_{i}, \gamma_{i}$ for $1 \leq i \leq j$ by

$$
\alpha_{j}=\kappa_{j}^{-1}, \quad \beta_{j}=\kappa_{j}-k, \quad \gamma_{j}=1+\kappa_{j}-k,
$$

and for $i=j-1, \ldots, 1$, successively by means of (4.7). Then we find that $\phi$ and $\lambda_{s}^{\prime}$ satisfy (4.8)-(4.10), and again we are able to establish new permissible exponents by iterating this and related procedures.

(ii) Process $N_{j}^{s}$. Next consider the iterative scheme $\left(\mathrm{M}_{1}\right)$ above. Suppose that the conditions of Lemma 5.7( $\left.\mathrm{I}^{\prime}\right)$ hold with $u=s-j-1$. Then we find that $\lambda_{s}^{\prime}$ and $\phi$ are determined by (5.28)-(5.34). Meanwhile, when the conditions of Lemma 5.7( $\left.\mathrm{II}^{\prime}\right)$ hold with $u=s-j-1$, one finds instead that $\lambda_{s}^{\prime}$ and $\phi$ are determined by (5.35), (5.36) and (4.7)-(4.10). In either case 
we are able to establish new permissible exponents by iterating these and similar procedures.

6. Waring's problem. We defer announcing the permissible exponents obtained through our methods to Sections 9 to 22, but pause here to indicate how Theorem 1.1 may be established by means of the latter exponents. We require the following theorem, which is essentially a consequence of Corollary 1 to Theorem 4.2 of Wooley [22] and Theorem 5.1 of [22].

THEOREM 6.1. Let $s, t$ and $w$ be natural numbers satisfying $2 s \geq k+1$, and suppose that $\Delta_{n}(n=s, t, w)$ are admissible exponents. Define

$$
\sigma(k)=\frac{k-\Delta_{t}-\Delta_{s} \Delta_{w}}{2\left(s\left(k+\Delta_{w}-\Delta_{t}\right)+t w\left(1+\Delta_{s}\right)\right)}
$$

and

$$
\lambda(k)=\frac{s\left(k-\Delta_{t}\right)+t w \Delta_{s}}{s\left(k+\Delta_{w}-\Delta_{t}\right)+t w\left(1+\Delta_{s}\right)} .
$$

Suppose that

$$
1 / 2<\lambda(k)<1-\sigma(k) .
$$

Then for each natural number $v$ with $v \geq k$, and each admissible exponent $\Delta_{v}$, we have

$$
G(k) \leq \max \left\{2 v+1+\left[\frac{\Delta_{v}}{\sigma(k)}\right], 4 k\right\} .
$$

We note that some minor modifications to the argument of the proof of Theorem 5.1 of [22] will be required in order to account for the use of the inequality (2.12) in place of the mean value

$$
\int_{0}^{1}\left|F_{0}(\alpha)^{2} f_{0}(\alpha)^{2 s}\right| d \alpha \ll P^{\lambda_{s+1}+\varepsilon} .
$$

However, the replacement of the generating functions $f_{0}(\alpha)$ in the latter by $f_{0}^{+}(\alpha)$ in the former causes no technical problems, and affects only the singular integral in the asymptotic formula resulting from the application of the Hardy-Littlewood method. This singular integral, moreover, is easily bounded below by the expected quantity using only the methods of Chapter 2 of Vaughan [15], and so the desired conclusion follows with little difficulty.

In order to establish Theorem 1.1, one merely optimises the choice of $\sigma(k)$ through appropriate choices of $s, t, w$, and then one optimises the upper bound (6.4) for $G(k)$ through a suitable choice of $v$.

7. Distribution of $\alpha n^{k}$ modulo 1. We turn our attention now to the proofs of Theorems 1.2 and 1.3. Note first that the discussion of Section 6 
of Wooley [22] leading to the proof of Theorem 1.2 of [22] establishes the following theorem.

TheOREM 7.1. Let $k \geq 4$, and suppose that $\sigma(k)$ and $\lambda(k)$ are defined as in (6.1) and (6.2), and satisfy (6.3). Let $\alpha \in \mathbb{R}$ and $\varepsilon>0$. Then there is a real number $N(\varepsilon, k)$ with the property that whenever $N \geq N(\varepsilon, k)$, one has

$$
\min _{1 \leq n \leq N}\left\|\alpha n^{k}\right\| \leq N^{\varepsilon-\sigma(k)} .
$$

Thus the work expended in establishing Theorem 1.1 already yields the conclusion of Theorem 1.2.

The proof of Theorem 1.3 is a little more involved, though in principle this follows the argument of the proof of Theorem 1.1 of Wooley [20] in essentially all details.

TheOREM 7.2. Let $k$ be a natural number with $7 \leq k \leq 20$, and let $\alpha \in \mathbb{R}$ and $\varepsilon>0$. Suppose that $s$ is a natural number with $1 \leq s \leq k$, and that $\Delta_{s}$ is an admissible exponent derived through the methods described in Sections 9-22. Define

$$
\tau(k)=\frac{k-2 \Delta_{s}}{4 s^{2}-1} .
$$

Then there are infinitely many natural numbers $n$ satisfying $\left\|\alpha n^{k}\right\| \leq n^{\varepsilon-\tau(k)}$.

Of course, the exponent claimed in the statement of Theorem 7.2 is valid in far greater generality, but our proof is much simplified by restricting to the methods of this paper. When $P$ and $H$ are large real numbers, denote by $U_{s}(P, H, R)$ the number of solutions of the diophantine equation

$$
h_{1} x_{1}^{k}+\ldots+h_{s} x_{s}^{k}=g_{1} y_{1}^{k}+\ldots+g_{s} y_{s}^{k}
$$

with

$$
1 \leq h_{i}, g_{i} \leq H \quad \text { and } \quad x_{i}, y_{i} \in \mathcal{A}(P, R) \quad(1 \leq i \leq s) .
$$

In order to prove Theorem 7.2, we follow the argument of the proof of Lemma 4.2 of [20], together with the argument of the proof of Theorem 1.1 of that paper (described at the end of Section 4 of [20]). Thus we find that the conclusion of Theorem 7.2 will follow provided only that we establish that when $s$ is a natural number with $1 \leq s \leq k$, and $\lambda_{s}$ is a permissible exponent, then

$$
U_{s}(P, H, R) \ll H^{2 s-1+\varepsilon} P^{\lambda_{s}+\varepsilon} .
$$

A program for establishing such bounds is described in Section 3 of [20], but in light of subsequent developments we feel obliged to outline some of the necessary steps so far as the application at hand is concerned. Since a full account of such a proof would be costly in terms of space, we will be economical in the details by referring frequently to earlier work. 
When $1 \leq j \leq k$, we write

$$
\mathfrak{f}_{j}(\alpha)=\sum_{1 \leq g \leq H} f_{j}(g \alpha) \text { and } \mathfrak{F}_{j}(\alpha)=\sum_{1 \leq g \leq H} F_{j}(g \alpha),
$$

and we note that by orthogonality, one has

$$
U_{s}(P, H, R)=\int_{0}^{1}\left|\mathfrak{f}_{0}(\alpha)\right|^{2 s} d \alpha .
$$

We will refer to an exponent $\lambda_{s}$ as derived whenever the inequality (7.2) holds. Our aim is to show, at least when $1 \leq s \leq k$, that the exponent $\lambda_{s}$ is derived whenever $\lambda_{s}$ is also a permissible exponent stemming from the methods described herein. In this context, we note that $\lambda_{s}=2 s-1$ is always a derived exponent. For suppose that $\mathbf{g}, \mathbf{h}, \mathbf{x}, \mathbf{y}$ is any solution of the equation (7.1) counted by $U_{s}(P, H, R)$. For each fixed choice of $h_{i}, x_{i}$ $(2 \leq i \leq s)$ and $g_{j}, y_{j}(1 \leq j \leq s)$, an elementary estimate for the divisor function shows that there are at most $O\left((H P)^{\varepsilon}\right)$ possible choices for $h_{1}$ and $x_{1}$, whence

$$
U_{s}(P, H, R) \ll(H P)^{2 s-1+\varepsilon} .
$$

Observe next that as a consequence of Lemma 3.4 of [20], and the argument of the proof of Lemma 3.1 of Wooley [19] (see, in particular, equation (3.7)), one has for $0 \leq j \leq k-1$,

$$
\begin{aligned}
& \int_{0}^{1}\left|\mathfrak{F}_{j}(\alpha)^{2} \mathfrak{f}_{j}(\alpha)^{2 s}\right| d \alpha \\
& \ll P^{\varepsilon} H^{1+\varepsilon} \widetilde{H}_{j} \widetilde{M}_{j} M_{j+1}^{2 s-1} \\
& \quad \times\left(H P \widetilde{H}_{j} \widetilde{M}_{j+1} U_{s}\left(Q_{j}, H, R\right)+\int_{0}^{1}\left|\widetilde{F}_{j+1}(\alpha) \mathfrak{f}_{j+1}(\alpha)^{2 s}\right| d \alpha\right) .
\end{aligned}
$$

Thus, whenever $\left(\lambda_{s}\right)$ is an existing sequence of derived exponents, one obtains the following analogues of Lemmata 2.1 and 2.2 above by essentially identical arguments.

LEMMA 7.3. We have

$$
\begin{aligned}
& \int_{0}^{1}\left|\mathfrak{F}_{0}(\alpha)^{2} \mathfrak{f}_{0}(\alpha)^{2 s}\right| d \alpha \\
& \ll(H P)^{\varepsilon} M_{1}^{2 s-1}\left(H^{2 s+1} P M_{1} Q_{1}^{\lambda_{s}}+H \int_{0}^{1}\left|\mathfrak{F}_{1}(\alpha) \mathfrak{f}_{1}(\alpha)^{2 s}\right| d \alpha\right) .
\end{aligned}
$$


Lemma 7.4. Whenever $0<t<2 s$ and $1 \leq j \leq k-1$, we have

$$
\int_{0}^{1}\left|\mathfrak{F}_{j}(\alpha) \mathfrak{f}_{j}(\alpha)^{2 s}\right| d \alpha \ll(H P)^{\varepsilon}\left(H^{2 t-1} Q_{j}^{\lambda_{t}}\right)^{1 / 2}\left(H \widetilde{H}_{j} \widetilde{M}_{j} M_{j+1}^{4 s-2 t-1} T_{j+1}\right)^{1 / 2},
$$

where

$$
T_{j+1}=P \widetilde{H}_{j} \widetilde{M}_{j+1} H^{4 s-2 t} Q_{j+1}^{\lambda_{2 s-t}}+\int_{0}^{1}\left|\mathfrak{F}_{j+1}(\alpha) \mathfrak{f}_{j+1}(\alpha)^{4 s-2 t}\right| d \alpha .
$$

The reader may wish to compare Lemma 7.4 with Lemma 3.5 of [20], which considers the special case with $s=t$.

Now observe from the tables in Sections 9-22 that for $7 \leq k \leq 20$ and $1 \leq s \leq k$, the iterative procedures described herein always terminate with processes of type $A_{j}^{s, l}$ or $B_{j, t}^{s, l}(l=1,2)$. A modicum of contemplation within the discussion of Section 4 above therefore leads one to the conclusion that the claimed bound (7.2) will follow, for any permissible exponent $\lambda_{s}$ produced by the methods of this paper for $1 \leq s \leq k$, so long as we are able to establish the estimates contained in the following lemmata.

Lemma 7.5. With the hypotheses of the statement of Theorem 3.4 for $1 \leq l \leq k-2$, subject to (Ia) or (Ib), one has

$$
\int_{0}^{1}\left|\mathfrak{F}_{j}(\alpha)\right|^{2^{l}} d \alpha \ll H^{2^{l}-1+\varepsilon} P^{2^{l}-l+\varepsilon} \widetilde{M}_{j}^{2^{l}-1} \widetilde{H}_{j}^{2^{l}-1},
$$

subject to (Ic), one has

$$
\int_{0}^{1}\left|\mathfrak{F}_{j}(\alpha)\right|^{2^{l}} d \alpha \ll H^{2^{l}-1+\varepsilon} P^{2^{l}-l+\varepsilon} \widetilde{M}_{j}^{2^{l}-1+\sigma} \widetilde{H}_{j}^{2^{l}-1},
$$

subject to (II), one has

$$
\int_{0}^{1}\left|\mathfrak{F}_{j}(\alpha)\right|^{2^{l}} d \alpha \ll H^{2^{l}-1+\varepsilon} P^{2^{l}-l-1 / 3+\varepsilon} \widetilde{M}_{j}^{2^{l}} \widetilde{H}_{j}^{2^{l}-1},
$$

and subject to (III), one has

$$
\int_{0}^{1}\left|\mathfrak{F}_{j}(\alpha)\right|^{2^{l}} d \alpha \ll H^{2^{l}-1+\varepsilon} P^{2^{l}-l+\varepsilon} \widetilde{M}_{j}^{2^{l}} \widetilde{H}_{j}^{2^{l}-1} .
$$

Lemma 7.6. Suppose that $2 \leq l \leq k-2$. Then

$$
\int_{0}^{1}\left|\mathfrak{F}_{k-l}(\alpha)\right|^{2^{l}} d \alpha \ll H^{2^{l}-1+\varepsilon} P^{2^{l}-l+\varepsilon} \widetilde{M}_{k-l}^{2^{l}} \widetilde{H}_{k-l}^{2^{l}-1} .
$$


In order to establish Lemmata 7.5 and 7.6, we note that in the diophantine equations underlying the mean values

$$
\int_{0}^{1}\left|\mathfrak{F}_{j}(\alpha)\right|^{2^{l}} d \alpha,
$$

the equations differ from those underlying $R_{j}^{(s)}(P ; \phi)$, defined in (3.1), only in so far as an additional linear variable in the interval $[1, H]$ occurs as a coefficient of each polynomial $\Psi_{j}$. Consequently, on following the argument of the proof of Lemma 3.1 above, we find that

$$
\begin{aligned}
\int_{0}^{1}\left|\mathfrak{F}_{j}(\alpha)\right|^{2^{l+1}} d \alpha \ll & P^{2^{l}-1}\left(H \widetilde{H}_{j} \widetilde{M}_{j}\right)^{2^{l}} \int_{0}^{1}\left|\mathfrak{F}_{j}(\alpha)\right|^{2^{l}} d \alpha \\
& +P^{2^{l}-l-1}\left(H \widetilde{H}_{j} \widetilde{M}_{j}\right)^{2^{l}-1}\left(S^{\prime} \int_{0}^{1}\left|\mathfrak{F}_{j}(\alpha)\right|^{2^{l+1}} d \alpha\right)^{1 / 2}
\end{aligned}
$$

where $S^{\prime}$ denotes the number of solutions of the equation

$$
h \Psi_{j, l}(z ; \mathbf{h} ; \mathbf{m} ; \mathbf{u})=g \Psi_{j, l}(w ; \mathbf{g} ; \mathbf{n} ; \mathbf{v}),
$$

with the polynomials $\Psi_{j, l}$ as in the proof of Lemma 3.1, and with the variables in the same ranges, save that $1 \leq g, h \leq H$. Then by a divisor estimate argument paralleling the start of the proof of Lemma 3.2 of [18], we find that

$$
\begin{aligned}
\int_{0}^{1}\left|\mathfrak{F}_{j}(\alpha)\right|^{2^{l+1}} d \alpha \ll & P^{2^{l}-1}\left(H \widetilde{H}_{j} \widetilde{M}_{j}\right)^{2^{l}} \int_{0}^{1}\left|\mathfrak{F}_{j}(\alpha)\right|^{2^{l}} d \alpha \\
& +P^{2^{l+1}-2 l-2}\left(H \widetilde{H}_{j} \widetilde{M}_{j}\right)^{2^{l+1}-2} H^{1+\varepsilon} R_{j+l}^{(1)}(P ; \boldsymbol{\phi}, \mathbf{0}) .
\end{aligned}
$$

On considering the underlying diophantine equations, the bounds claimed in Lemmata 7.5 and 7.6 now follow by an inductive argument similar to that employed in the proofs of Theorems 3.4 and 3.5.

This completes the proof of Theorem 7.2.

8. Preliminary discussion of computations. By employing a computer to optimise the use of the methods described in Sections $2-5$ of this paper, one derives an upper bound for a sequence $\left(\lambda_{s}\right)$ of permissible exponents. In the tables presented in Sections 9-22, we record for each value of $k$ the permissible exponents thus derived, together with the process yielding these exponents towards the end of the iteration process. Naturally, the conditions necessary for the application of the latter process may not initially hold. Under such circumstances, we begin by applying simpler, more robust, versions of such processes. Thus, for example, case (III) of Theorem 3.4 implies that processes $A$ and $B$ may always be applied with $\tau=1$. In order to give some indication of the parameters $\phi$ arising in these iterative 
processes, we record also the values of $\phi_{1}, \phi_{j}, \sum_{i=1}^{j} \phi_{i}$ (when $j>1$ ), and when $j \geq 3$ we record also the value of

$$
\phi_{s}^{*}=\max _{3 \leq I \leq j}\left(\sum_{i=1}^{I} \phi_{i}+k\left(\phi_{I-1}+\phi_{I}\right)\right),
$$

corresponding to each process involving $j$ differencing operations. Adjacent to the table, we discuss any issues pertaining to the applicability of iterative processes in the light of the conditions associated with the use of Theorems 3.4, 3.5 and Lemma 5.7. In particular, we note that the parameter $\chi_{j, l}$ is zero throughout unless otherwise indicated. Recorded values for $\lambda_{s}$ and $\phi$ are upper bounds, computations having been performed in double precision arithmetic.

Following the primary table, we record also the values of $\sigma(k), \tau(k)$ and $G(k)$ (for $k \geq 9$ ) stemming from Theorems 6.1 and 7.2, the values of the former quantities recorded being lower bounds. We provide a parenthetic indication of the relevant parameters employed in the derivation of these values.

Note that the method of the proof of Theorem 5.1 of Wooley [22] shows that whenever $\Delta_{v}$ is an admissible exponent, then for

$$
s \geq v+\left[\frac{\Delta_{v}}{2 \sigma(k)}\right]+1,
$$

one has

$$
\int_{0}^{1}\left|f_{0}(\alpha)\right|^{2 s} d \alpha \ll P^{2 s-k}
$$

whence $\lambda_{s}=2 s-k$ is a permissible exponent.

A final word is in order concerning the application of the processes $M_{j}^{s}$ and $N_{j}^{s}$. When calculating a permissible exponent $\lambda_{s}$ by means of Lemma 5.7 , one frequently encounters conditions involving admissible exponents $\Delta_{u}$ with $u$ substantially larger than $s$. Thus it is useful to prepare preliminary estimates by applying process $M_{j}^{s}$ throughout, where $j$ is sufficiently small that the condition (B) in the simple variants (5.25)-(5.27) is applicable. Since the conditions $\left(D_{1}\right)$ and $\left(D_{2}\right)$ are easy to check computationally, one obtains in this manner reasonably strong permissible exponents $\lambda_{s}$ with $s$ exceeding some suitable natural number $s_{0}$. Equipped with these preliminary bounds, we may subsequently refine the iterative procedures so as to attain the exponents claimed in the primary tables. Comments clarifying this process are included in each section.

We conclude by discussing a final simple process not without interest.

Process $D^{s}$. Suppose that $s \geq k$ and $t$ is a natural number. Then whenever $\lambda_{s}$ is a permissible exponent, then also the exponent $\lambda_{s+t}^{\prime}$ is permissible, 
where

$$
\lambda_{s+t}^{\prime}=\max \left\{\lambda_{s}+2 t(1-\sigma(k)), 2 s+2 t-k\right\},
$$

and here $\sigma(k)$ is the exponent arising in the statement of Theorem 6.1. In order to establish this claim, we adapt the argument of the proof of Theorem 5.1 of Wooley [22]. Write

$$
f(\alpha)=\sum_{x \in \mathcal{A}(P, R)} e\left(\alpha x^{k}\right) \quad \text { and } \quad g(\alpha)=\sum_{1 \leq x \leq P} e\left(\alpha x^{k}\right) .
$$

Let $\mathfrak{m}$ denote the set of real numbers $\alpha \in[0,1)$ with the property that, whenever $a \in \mathbb{Z}, q \in \mathbb{N},(a, q)=1$ and $|q \alpha-a| \leq P^{1-k}$, one has $q>P$. Then as in the proof of Theorem 5.1 of [22], one has

$$
\begin{aligned}
\int_{\mathfrak{m}}\left|g(\alpha)^{2} f(\alpha)^{2 s+2 t-2}\right| d \alpha & \ll\left(\sup _{\alpha \in \mathfrak{m}}|f(\alpha)|\right)^{2 t} \int_{0}^{1}\left|g(\alpha)^{2} f(\alpha)^{2 s-2}\right| d \alpha \\
& \ll\left(P^{1-\sigma(k)+\varepsilon}\right)^{2 t} P^{\lambda_{s}+\varepsilon} .
\end{aligned}
$$

On the other hand, provided that $s \geq k$, one may apply a standard pruning argument, of the type described in Section 5 of Vaughan [13], to show that

$$
\int_{\mathfrak{M}}\left|g(\alpha)^{2} f(\alpha)^{2 s+2 t-2}\right| d \alpha \ll P^{2 s+2 t-k} .
$$

By considering the underlying diophantine equations, the claimed conclusion follows on noting that

$$
\int_{0}^{1}|f(\alpha)|^{2 s+2 t} d \alpha \ll \int_{0}^{1}\left|g(\alpha)^{2} f(\alpha)^{2 s+2 t-2}\right| d \alpha .
$$

As a consequence of the process $D^{s}$, we may restrict attention to those $s$ for which the processes $A, B, N$ or $M$ demonstrate that the permissible exponent $\lambda_{s}$ satisfies

$$
\lambda_{s}<\lambda_{s-1}+2(1-\sigma(k)),
$$

for all permissible exponents $\lambda_{s-1}$.

9. Permissible exponents for seventh powers. Following the computational procedure outlined in Section 8, we obtain the permissible exponents recorded in the table below. The exponents $\lambda_{s}$ listed in the table for $3 \leq s \leq 12$ are identical with those on p. 237 of [18], and indeed for $s=3,4$, these exponents were established earlier by Vaughan [14]. We remark that in this range of $s$, one may take $\tau_{j, l}=0(l=1,2)$ throughout (see [18] for details). Our computations for $s \geq 13$ depend on first obtaining preliminary estimates by applying the process $M_{2}^{s}$ throughout (noting (5.25) and checking $\left(\mathrm{D}_{1}\right)$ or $\left.\left(\mathrm{D}_{2}\right)\right)$. In this way we obtain the preliminary permissible exponents 
Table of permissible exponents for $k=7$

\begin{tabular}{ccccccc}
\hline$s$ & Process & $\lambda_{s}$ & $\phi_{1}$ & $\phi_{j}$ & $\sum_{i=1}^{j} \phi_{i}$ & $\phi_{s}^{*}$ \\
\hline 3 & $B_{1,4}^{3,2}$ & 3.0639191 & 0.03195955 & & & \\
4 & $A_{1}^{4,2}$ & 4.2641175 & 0.06818557 & & & \\
5 & $B_{2,5}^{5,2}$ & 5.5891167 & 0.08699398 & 0.0355 & 0.1225 & \\
6 & $A_{2}^{6,2}$ & 7.0143820 & 0.09641272 & 0.0694 & 0.1658 & \\
7 & $B_{3,6}^{7,2}$ & 8.5410894 & 0.10564538 & 0.0406 & 0.2343 & 1.1347 \\
8 & $A_{3}^{8,2}$ & 10.1526323 & 0.11202654 & 0.0691 & 0.2800 & 1.4554 \\
9 & $B_{4,8}^{9,2}$ & 11.8469485 & 0.11873997 & 0.0416 & 0.3577 & 1.6983 \\
10 & $A_{4}^{10,2}$ & 13.6055676 & 0.12329153 & 0.0661 & 0.4030 & 1.8315 \\
11 & $A_{4}^{11,2}$ & 15.4242973 & 0.12803790 & 0.0859 & 0.4429 & 1.9600 \\
12 & $A_{4}^{12,2}$ & 17.2932208 & 0.13214156 & 0.1027 & 0.4781 & 2.0785 \\
13 & $M_{4}^{13}$ & 19.1925374 & 0.13409068 & 0.1072 & 0.4919 & 2.1387 \\
14 & $M_{4}^{14}$ & 21.1139297 & 0.13535033 & 0.1072 & 0.4957 & 2.1606 \\
15 & $M_{3}^{15}$ & 23.0528848 & 0.13635572 & 0.1226 & 0.3903 & 2.1673 \\
16 & $M_{2}^{16}$ & 25.0105382 & 0.13784908 & 0.1327 & 0.2706 & \\
$s \geq 17$ & $D^{s}$ & $2 s-7$ & & & & \\
\hline$\sigma(7)=0.017475$ & $(s=14, t=5, w=8)$, & $\tau(7)=0.020777$ & $(s=6)$ \\
\hline
\end{tabular}

$\lambda_{13}=19.211, \quad \lambda_{14}=21.127, \quad \lambda_{15}=23.073, \quad \lambda_{16}=25.019$,

and $\lambda_{s}=2 s-7$ for $s \geq 17$. Equipped with these preliminary bounds, we refine our procedure as indicated in the table. One may computationally check the validity of the appropriate case of Lemma 5.7 as follows.

(a) $s=13,14$. With process $M_{4}^{s}$, one finds that Lemma 5.7(I) holds with $u=s-5$ by virtue of conditions $\left(\mathrm{D}_{1}\right),\left(\mathrm{C}_{1}\right),\left(\mathrm{C}_{2}\right),\left(\mathrm{C}_{3}\right)$.

(b) $s=15$. With process $M_{3}^{15}$, one finds that Lemma 5.7(I) holds with $u=11$ by virtue of conditions $\left(\mathrm{D}_{1}\right),(\mathrm{B})$.

(c) $s=16$. With process $M_{2}^{16}$, one finds that Lemma 5.7(I) holds with $u=13$ by virtue of conditions $\left(\mathrm{D}_{1}\right),(\mathrm{B})$.

(d) $s \geq 17$. One finds that process $D^{s}$ applies.

10. Permissible exponents for eighth powers. Following the computational procedure outlined in Section 8, we obtain the permissible exponents recorded in the table below. The exponents $\lambda_{s}$ listed in the table improve on those provided by $[16,18]$ for $3 \leq s \leq 20$. Broadly speaking one can follow the discussion of [18] for $3 \leq s \leq 14$, though the improvements contained in Theorem 3.4 lead to sharper estimates, and permit a slightly more powerful iterative process. In particular, one may take $\tau_{j, l}=0$ $(l=1,2)$ for $3 \leq s \leq 12$, and $\tau_{5,2}=0.002$ for $s=13,14$ (see Section 11 
Table of permissible exponents for $k=8$

\begin{tabular}{ccccccc}
\hline$s$ & Process & $\lambda_{s}$ & $\phi_{1}$ & $\phi_{j}$ & $\sum_{i=1}^{j} \phi_{i}$ & $\phi_{s}^{*}$ \\
\hline 3 & $B_{1,4}^{3,2}$ & 3.0469787 & 0.02348931 & & & \\
4 & $A_{1}^{4,2}$ & 4.2164889 & 0.05740233 & & & \\
5 & $B_{2,2}^{5,2}$ & 5.4915710 & 0.07270549 & 0.0222 & 0.0949 & \\
6 & $A_{2}^{6,2}$ & 6.8566563 & 0.08097841 & 0.0563 & 0.1373 & \\
7 & $B_{3,7}^{7,2}$ & 8.3105992 & 0.08825831 & 0.0264 & 0.1881 & 0.9864 \\
8 & $A_{3}^{8,2}$ & 9.8428621 & 0.09355343 & 0.0559 & 0.2322 & 1.3410 \\
9 & $A_{3}^{9,2}$ & 11.4529104 & 0.09907986 & 0.0750 & 0.2646 & 1.5885 \\
10 & $A_{4}^{10,2}$ & 13.1283069 & 0.10315980 & 0.0550 & 0.3379 & 1.7214 \\
11 & $A_{4}^{11,2}$ & 14.8664781 & 0.10742204 & 0.0741 & 0.3750 & 1.8485 \\
12 & $A_{5}^{12,2}$ & 16.6561197 & 0.11069450 & 0.0528 & 0.4510 & 1.9401 \\
13 & $A_{5}^{13,2}$ & 18.4901012 & 0.11356143 & 0.0686 & 0.4849 & 2.0172 \\
14 & $A_{5}^{14,2}$ & 20.3623532 & 0.11614698 & 0.0820 & 0.5156 & 2.0883 \\
15 & $M_{5}^{15}$ & 22.2661078 & 0.11832893 & 0.0938 & 0.5428 & 2.1728 \\
16 & $M_{5}^{16}$ & 24.1891161 & 0.11934590 & 0.0938 & 0.5484 & 2.2022 \\
17 & $M_{5}^{17}$ & 26.1294925 & 0.12039309 & 0.0938 & 0.5527 & 2.2249 \\
18 & $M_{4}^{18}$ & 28.0833353 & 0.12119204 & 0.1075 & 0.4620 & 2.2385 \\
19 & $M_{4}^{19}$ & 30.0473193 & 0.12176644 & 0.1075 & 0.4638 & 2.2447 \\
20 & $D^{20}$ & 32.0186056 & & & & \\
\hline$\geq 21$ & $D^{s}$ & $2 s-8$ & & & & \\
\hline$\sigma(8)=0.014356$ & $(s=16, t=6, w=10)$, & $\tau(8)=0.017327$ & $(s=7)$ \\
\hline
\end{tabular}

of [18] for details). Our computations for $s \geq 15$ depend on first obtaining preliminary estimates by applying the process $M_{3}^{s}$ for $16 \leq s \leq 20$, and $M_{2}^{s}$ for $s \geq 21$ (noting (5.26) and checking $\left(\mathrm{D}_{1}\right)$ or $\left(\mathrm{D}_{2}\right)$ ). In this way we obtain the preliminary permissible exponents

$$
\begin{aligned}
& \lambda_{15}=22.282, \quad \lambda_{16}=24.206, \quad \lambda_{17}=26.143, \quad \lambda_{18}=28.098, \\
& \lambda_{19}=30.061, \quad \lambda_{20}=32.031, \quad \lambda_{21}=34.010,
\end{aligned}
$$

and $\lambda_{s}=2 s-8$ for $s \geq 22$. Equipped with these preliminary bounds, we refine our procedure as indicated in the table. One may computationally check the validity of the appropriate case of Lemma 5.7 as follows.

(a) $15 \leq s \leq 17$. With process $M_{5}^{s}$, one finds that Lemma 5.7(I) holds with $u=s-6$ by virtue of conditions $\left(\mathrm{D}_{1}\right),\left(\mathrm{C}_{1}\right),\left(\mathrm{C}_{2}\right),\left(\mathrm{C}_{3}\right)$.

(b) $s=18,19$. With process $M_{4}^{s}$, one finds that Lemma 5.7(I) holds with $u=s-5$ by virtue of conditions $\left(\mathrm{D}_{1}\right),\left(\mathrm{C}_{1}\right),\left(\mathrm{C}_{2}\right),\left(\mathrm{C}_{3}\right)$.

(c) $s \geq 20$. One finds that process $D^{s}$ applies. 
11. Permissible exponents for ninth powers. Following the computational procedure outlined in Section 8 , we obtain the permissible exponents recorded in the table below. The exponents $\lambda_{s}$ listed in the table improve those provided by [18] for $5 \leq s \leq 25$. For $s=3,4$, the exponents recorded in the table were established earlier by Vaughan [14]. Broadly speaking, we may again follow the discussion of [18] for $5 \leq s \leq 17$, though the improvements contained in Theorem 3.4 lead to sharper estimates, and permit slightly more powerful processes. We note in particular that when $k \geq 9$ and $1 \leq j \leq 3$, then in view of the inequality $0 \leq \phi_{i} \leq 1 / k(1 \leq i \leq j)$, one has

$$
\phi_{1}+\ldots+\phi_{j} \leq 3 / k \leq 1 / 3 .
$$

Thus, for $1 \leq j \leq 3$, the condition $(\delta)$ of Theorem 3.4 is automatically satisfied. Combining the latter observation with the methods of [18], it follows that one may take $\tau_{j, l}=0(l=1,2)$ for $3 \leq s \leq 11$ (in which interval our methods make use of a choice of $j$ with $1 \leq j \leq 4$ ). When $j=5$ and 6 , it follows from Theorem 3.4(Ib) case (iii) that one may take $\tau_{j, l}=0(l=1,2)$ provided only that

$$
\sum_{i=1}^{I} \phi_{i}+9\left(\phi_{I-1}+\phi_{I}\right) \leq 2
$$

when $I=3,4,5$. The computational verification of this inequality leads to the conclusion that one may take $\tau_{j, l}=0(l=1,2)$ also for $12 \leq s \leq 14$. Finally, when $j=6$ and $15 \leq s \leq 17$, it follows as in Section 11 of [18] that one may take $\tau_{j, l}=0.002565(l=1,2)$ whenever $\phi_{1} \leq 0.107131$.

As in the previous cases, our computations for $s \geq 18$ depend on first obtaining preliminary estimates by applying the process $M_{4}^{s}$ for $18 \leq s \leq 23$ (noting (5.26) and checking $\left(\mathrm{D}_{1}\right)$ or $\left(\mathrm{D}_{2}\right)$ ), and $D^{s}$ for $s \geq 24$. In this way we obtain the preliminary permissible exponents

$$
\begin{array}{lll}
\lambda_{18}=27.260, & \lambda_{19}=29.199, & \lambda_{20}=31.150, \\
\lambda_{21}=33.120, & \lambda_{22}=35.080, & \lambda_{23}=37.055,
\end{array}
$$

and by virtue of the preliminary exponent

$$
\sigma(9)=0.01212 \quad(s=19, t=6, w=12),
$$

we have also

$$
\lambda_{s}=\max \{2 s-9,37.055+2(s-23)(1-0.01212)\}
$$

for $s>23$. Equipped with these preliminary bounds, we refine our procedure as indicated in the table. One may computationally check the validity of the appropriate case of Lemma 5.7 as follows.

(a) $s=18,19$. With process $M_{6}^{s}$, one finds that Lemma 5.7(I) holds with $u=s-7$, by virtue of conditions $\left(\mathrm{D}_{1}\right),\left(\mathrm{C}_{1}\right),\left(\mathrm{C}_{2}\right),\left(\mathrm{C}_{3}\right)$. 
Table of permissible exponents for $k=9$

\begin{tabular}{ccccccc}
\hline$s$ & Process & $\lambda_{s}$ & $\phi_{1}$ & $\phi_{j}$ & $\sum_{i=1}^{j} \phi_{i}$ & $\phi_{s}^{*}$ \\
\hline 3 & $B_{1,4}^{3,2}$ & 3.0358052 & 0.01790259 & & & \\
4 & $A_{1}^{4,2}$ & 4.1822894 & 0.04941786 & & & \\
5 & $B_{2,6}^{5,2}$ & 5.4197057 & 0.06218814 & 0.0116 & 0.0737 & \\
6 & $B_{2,6}^{6,2}$ & 6.7383084 & 0.06955946 & 0.0463 & 0.1158 & \\
7 & $A_{2}^{7,2}$ & 8.1356346 & 0.07551302 & 0.0626 & 0.1381 & \\
8 & $B_{3,7}^{8,2}$ & 9.6039271 & 0.07985390 & 0.0450 & 0.1956 & 1.2367 \\
9 & $A_{3}^{9,2}$ & 11.1425026 & 0.08420410 & 0.0620 & 0.2231 & 1.4729 \\
10 & $A_{4}^{10,2}$ & 12.7463430 & 0.08805551 & 0.0451 & 0.2870 & 1.6267 \\
11 & $A_{4}^{11,2}$ & 14.4105835 & 0.09157319 & 0.0625 & 0.3194 & 1.7451 \\
12 & $A_{5}^{12,2}$ & 16.1292111 & 0.09468812 & 0.0451 & 0.3870 & 1.8387 \\
13 & $A_{5}^{13,2}$ & 17.8959526 & 0.09741610 & 0.0607 & 0.4194 & 1.9155 \\
14 & $B_{6,11}^{14,2}$ & 19.7055987 & 0.09990639 & 0.0420 & 0.4882 & 1.9858 \\
15 & $A_{6}^{15,2}$ & 21.5507274 & 0.10189148 & 0.0553 & 0.5185 & 2.0414 \\
16 & $A_{6}^{16,2}$ & 23.4269614 & 0.10370526 & 0.0673 & 0.5468 & 2.1092 \\
17 & $A_{6}^{17,2}$ & 25.3292029 & 0.10524175 & 0.0775 & 0.5717 & 2.1717 \\
18 & $M_{6}^{18}$ & 27.2520471 & 0.10643130 & 0.0834 & 0.5885 & 2.2317 \\
19 & $M_{6}^{19}$ & 29.1901860 & 0.10724097 & 0.0834 & 0.5937 & 2.2558 \\
20 & $M_{5}^{20}$ & 31.1420569 & 0.10804665 & 0.0958 & 0.5163 & 2.2898 \\
21 & $M_{5}^{21}$ & 33.1033373 & 0.10852186 & 0.0958 & 0.5185 & 2.2962 \\
22 & $M_{5}^{22}$ & 35.0727119 & 0.10895936 & 0.0958 & 0.5203 & 2.3010 \\
$\geq 23$ & $D^{s}$ & & & & & \\
\hline & & $\sigma(9)=0.012183$ & $(s=19, t=6, w=12)$, & \\
& $\tau(9)=0.014871$ & $(s=8)$, & $G(9) \leq 50$ & $(v=22)$ & \\
\hline & & & & & &
\end{tabular}

(b) $20 \leq s \leq 22$. With process $M_{5}^{s}$, one finds that Lemma 5.7(I) holds with $u=s-6$, by virtue of conditions $\left(\mathrm{D}_{1}\right),\left(\mathrm{C}_{1}\right),\left(\mathrm{C}_{2}\right),\left(\mathrm{C}_{3}\right)$.

(c) $s \geq 23$. One finds that process $D^{s}$ applies.

12. Permissible exponents for tenth powers. Following the computational procedure outlined in Section 8 , we obtain the permissible exponents recorded in the table below. The exponents $\lambda_{s}$ listed in the table improve on those previously available for $s \geq 3$. Our strategy is similar to that described in previous sections. Note first that for $3 \leq s \leq 11$, the condition $(\delta)$ of Theorem 3.4 is satisfied. Since when $j=5,6$, one of the conditions $(\alpha)$ and $(\beta)$ of Theorem 3.4 is satisfied, and one of the conditions (i) and (iii) of Theorem 3.4 is also satisfied for $3 \leq s \leq 15$, we deduce that one may take $\tau_{j, l}=0(l=1,2)$ for $3 \leq s \leq 15$. 
Table of permissible exponents for $k=10$

\begin{tabular}{|c|c|c|c|c|c|c|}
\hline$s$ & Process & $\lambda_{s}$ & $\phi_{1}$ & $\phi_{j}$ & $\sum_{i=1}^{j} \phi_{i}$ & $\phi_{s}^{*}$ \\
\hline 3 & $B_{1,4}^{3,2}$ & 3.0281105 & 0.01405522 & & & \\
\hline 4 & $A_{1}^{4,2}$ & 4.1568114 & 0.04330611 & & & \\
\hline 5 & $B_{2,6}^{5,2}$ & 5.3659530 & 0.05441877 & 0.0042 & 0.0586 & \\
\hline 6 & $B_{2,6}^{6,2}$ & 6.6465971 & 0.06056135 & 0.0375 & 0.0981 & \\
\hline 7 & $A_{2}^{7,2}$ & 7.9967162 & 0.06540123 & 0.0525 & 0.1179 & \\
\hline 8 & $B_{3,7}^{8,2}$ & 9.4143540 & 0.06956822 & 0.0361 & 0.1670 & 1.1412 \\
\hline 9 & $A_{3}^{9,2}$ & 10.8945712 & 0.07291878 & 0.0516 & 0.1912 & 1.3731 \\
\hline 10 & $A_{4}^{10,2}$ & 12.4375675 & 0.07641992 & 0.0368 & 0.2472 & 1.5499 \\
\hline 11 & $A_{4}^{11,2}$ & 14.0371956 & 0.07929039 & 0.0525 & 0.2751 & 1.6556 \\
\hline 12 & $A_{5}^{12,2}$ & 15.6914013 & 0.08215771 & 0.0377 & 0.3350 & 1.7513 \\
\hline 13 & $A_{5}^{13,2}$ & 17.3943657 & 0.08460686 & 0.0524 & 0.3645 & 1.8239 \\
\hline 14 & $B_{6,11}^{14,2}$ & 19.1426918 & 0.08695770 & 0.0366 & 0.4265 & 1.8937 \\
\hline 15 & $A_{6}^{15,2}$ & 20.9303709 & 0.08892986 & 0.0502 & 0.4559 & 1.9503 \\
\hline 16 & $B_{6,13}^{16,3}$ & 22.7537459 & 0.09078376 & 0.0627 & 0.4843 & 2.0175 \\
\hline 17 & $A_{7}^{17,2}$ & 24.6071999 & 0.09230268 & 0.0456 & 0.5472 & 2.0724 \\
\hline 18 & $A_{7}^{18,2}$ & 26.4867878 & 0.09364492 & 0.0558 & 0.5724 & 2.1251 \\
\hline 19 & $A_{7}^{19,2}$ & 28.3886784 & 0.09480400 & 0.0647 & 0.5951 & 2.1845 \\
\hline 20 & $A_{7}^{20,2}$ & 30.3094873 & 0.09580462 & 0.0734 & 0.6171 & 2.2431 \\
\hline 21 & $N_{7}^{21}$ & 32.2449884 & 0.09653784 & 0.0750 & 0.6258 & 2.2800 \\
\hline 22 & $N_{7}^{22}$ & 34.1926960 & 0.09715085 & 0.0750 & 0.6306 & 2.3008 \\
\hline 23 & $M_{6}^{23}$ & 36.1509648 & 0.09770971 & 0.0863 & 0.5616 & 2.3354 \\
\hline 24 & $M_{6}^{24}$ & 38.1169804 & 0.09808226 & 0.0863 & 0.5639 & 2.3420 \\
\hline 25 & $M_{6}^{25}$ & 40.0895832 & 0.09841150 & 0.0863 & 0.5658 & 2.3469 \\
\hline 26 & $M_{6}^{26}$ & 42.0677228 & 0.09869813 & 0.0867 & 0.5681 & 2.3577 \\
\hline$s \geq 27$ & $D^{s}$ & & & & & \\
\hline \multicolumn{7}{|c|}{$\begin{array}{c}\sigma(10)=0.010569 \quad(s=22, t=7, w=13) \\
\tau(10)=0.013036 \quad(s=9), \quad G(10) \leq 59 \quad(v=26)\end{array}$} \\
\hline
\end{tabular}

In order to discuss permissible exponents for $16 \leq s \leq 20$, we apply case (Ic) of Theorem 3.4. In the notation of the latter theorem, we find from Section 22 that when $j=6$ or 7 and $8 \leq j+l \leq 9$, one has $J=1$, and hence one may take

$$
\begin{gathered}
\delta_{3}=0.0035377, \quad \delta_{4}=0.0372112, \quad \delta_{6}=0.2457501 \\
\delta_{7}=0.4042791 \quad \text { and } \quad \delta_{8}=0.5946271
\end{gathered}
$$

(note that Section 22 is independent of Sections 9-21). Thus we deduce that 
one may take

$$
\tau_{6, l}=\frac{1}{3} \delta_{3}<0.00118 \quad \text { whenever } \quad \phi_{1} \leq 0.097668 \quad(l=2,3),
$$

and that one may take

$$
\tau_{7, l}=\frac{1}{7}\left(\delta_{3}+\delta_{4}\right)<0.00583 \quad \text { whenever } \quad \phi_{1} \leq 0.095055 \quad(l=2),
$$

and otherwise, one may take

$$
\tau_{7, l}=\frac{1}{7} \delta_{7}<0.05776 .
$$

As in the previous cases, our computations for $s \geq 21$ depend on first obtaining preliminary estimates by applying the process $M_{5}^{s}$ for $21 \leq s \leq 26$ (noting (5.26) and checking $\left(\mathrm{D}_{1}\right)$ or $\left(\mathrm{D}_{2}\right)$ ), and $D^{s}$ for $s \geq 27$. In this way we obtain the preliminary permissible exponents

$$
\begin{array}{lll}
\lambda_{21}=32.249, & \lambda_{22}=34.198, & \lambda_{23}=36.156, \\
\lambda_{24}=38.122, & \lambda_{25}=40.094, & \lambda_{26}=42.072,
\end{array}
$$

and by virtue of the preliminary exponent

$$
\sigma(10)=0.01054 \quad(s=22, t=7, w=13)
$$

we have also

$$
\lambda_{s}=\max \{2 s-10,42.072+2(s-26)(1-0.01054)\}
$$

for $s>26$. Equipped with these preliminary bounds, we refine our procedure as indicated in the table. One may computationally check the validity of the appropriate case of Lemma 5.7 as follows.

(a) $s=21,22$. With process $N_{7}^{s}$, one finds that Lemma 5.7( (I') holds with $u=s-8$, by virtue of condition $\left(\mathrm{A}_{1}\right)$.

(b) $23 \leq s \leq 25$. With process $M_{6}^{s}$, one finds that Lemma 5.7(I) holds with $u=s-7$, by virtue of conditions $\left(\mathrm{D}_{1}\right),\left(\mathrm{C}_{1}\right),\left(\mathrm{C}_{2}\right),\left(\mathrm{C}_{3}\right)$.

(c) $s=26$. With process $M_{6}^{s}$, one finds that Lemma 5.7(II) holds with $u=19$, by virtue of conditions $\left(\mathrm{D}_{2}\right),\left(\mathrm{C}_{1}\right),\left(\mathrm{C}_{4}\right),\left(\mathrm{C}_{5}\right)$.

(d) $s \geq 27$. One finds that process $D^{s}$ applies.

13. Permissible exponents for eleventh powers. Following the computational procedure outlined in Section 8, we obtain the permissible exponents recorded in the table below. The exponents $\lambda_{s}$ listed in the table improve on those previously available for $s \geq 3$. We follow a similar path to that taken in previous sections. Note first that for $3 \leq s \leq 13$, the condition $(\delta)$ of Theorem 3.4 is satisfied. Since when $j=6,7$, one of the conditions $(\alpha),(\beta)$ and $(\gamma)$ of Theorem 3.4 is satisfied, and one of the conditions (i) and (iii) of Theorem 3.4 is also satisfied for $3 \leq s \leq 17$, we deduce that one may take $\tau_{j, l}=0(l=1,2,3)$ for $3 \leq s \leq 17$. 
Table of permissible exponents for $k=11$

\begin{tabular}{ccccccc}
\hline$s$ & Process & $\lambda_{s}$ & $\phi_{1}$ & $\phi_{j}$ & $\sum_{i=1}^{j} \phi_{i}$ & $\phi_{s}^{*}$ \\
\hline 3 & $B_{1,4}^{3,2}$ & 3.0221905 & 0.01109521 & & & \\
4 & $B_{1,5}^{4,2}$ & 4.1346363 & 0.03776126 & & & \\
5 & $A_{1}^{5,2}$ & 5.3216133 & 0.04837243 & & & \\
6 & $B_{2,6}^{6,2}$ & 6.5727501 & 0.05368021 & 0.0312 & 0.0848 & \\
7 & $A_{2}^{7,2}$ & 7.8855603 & 0.05763697 & 0.0449 & 0.1026 & \\
8 & $B_{3,8}^{8,2}$ & 9.2614256 & 0.06147175 & 0.0287 & 0.1442 & 1.0534 \\
9 & $A_{3}^{9,2}$ & 10.6937742 & 0.06416025 & 0.0434 & 0.1662 & 1.2881 \\
10 & $B_{4,9}^{10,2}$ & 12.1849727 & 0.06723014 & 0.0289 & 0.2140 & 1.4815 \\
11 & $A_{4}^{11,2}$ & 13.7292224 & 0.06964143 & 0.0441 & 0.2396 & 1.5798 \\
12 & $B_{5,10}^{12,2}$ & 15.3262982 & 0.07219102 & 0.0301 & 0.2917 & 1.6759 \\
13 & $A_{5}^{13,2}$ & 16.9712740 & 0.07435992 & 0.0446 & 0.3191 & 1.7442 \\
14 & $B_{6,11}^{14,2}$ & 18.6621448 & 0.07651920 & 0.0307 & 0.3745 & 1.8126 \\
15 & $A_{6}^{15,2}$ & 20.3940119 & 0.07837637 & 0.0441 & 0.4022 & 1.8679 \\
16 & $B_{6,13}^{16,3}$ & 22.1640483 & 0.08016212 & 0.0566 & 0.4295 & 1.9304 \\
17 & $A_{7}^{17,2}$ & 23.9674841 & 0.08168359 & 0.0422 & 0.4874 & 1.9847 \\
18 & $A_{7}^{18,2}$ & 25.8009828 & 0.08307973 & 0.0533 & 0.5133 & 2.0398 \\
19 & $A_{8}^{19,2}$ & 27.6607360 & 0.08429767 & 0.0378 & 0.5710 & 2.0979 \\
20 & $A_{8}^{20,2}$ & 29.5431019 & 0.08534127 & 0.0466 & 0.5939 & 2.1448 \\
21 & $A_{8}^{21,2}$ & 31.4450976 & 0.08625844 & 0.0548 & 0.6151 & 2.1947 \\
22 & $A_{8}^{22,2}$ & 33.3638548 & 0.08704554 & 0.0617 & 0.6340 & 2.2473 \\
23 & $N_{8}^{23}$ & 35.2968576 & 0.08772003 & 0.0682 & 0.6516 & 2.2983 \\
24 & $N_{8}^{24}$ & 37.2413126 & 0.08824091 & 0.0682 & 0.6568 & 2.3214 \\
25 & $M_{7}^{25}$ & 39.1958837 & 0.08872562 & 0.0786 & 0.5959 & 2.3658 \\
26 & $M_{7}^{26}$ & 41.1582991 & 0.08907859 & 0.0786 & 0.5988 & 2.3733 \\
27 & $M_{7}^{27}$ & 43.1274069 & 0.08938707 & 0.0786 & 0.6012 & 2.3800 \\
28 & $M_{7}^{28}$ & 45.1020502 & 0.08964222 & 0.0786 & 0.6031 & 2.3893 \\
29 & $N_{6}^{29}$ & 47.0818525 & 0.08990704 & 0.0848 & 0.5283 & 2.4164 \\
$\geq 30$ & $D^{s}$ & & & & & \\
\hline & & $\sigma(11)=0.009322$ & $(s=25, t=7, w=15)$, & \\
& $\tau(11)=$ & 0.011604 & $(s=10)$, & $G(11) \leq 67$ & $(v=29)$ & \\
\hline & & & & & & \\
\hline
\end{tabular}

In order to discuss permissible exponents for $18 \leq s \leq 22$, we apply case (Ic) of Theorem 3.4. In the notation of the latter theorem, we find from Section 23 that when $j=7$ or 8 and $l=2$, one has $J=1$, and hence one may take

$$
\delta_{3}=0.0025439, \quad \delta_{4}=0.0292912, \quad \delta_{8}=0.5257736 .
$$


Thus we deduce that one may take

$$
\tau_{7, l}=\frac{1}{7}\left(\delta_{3}+\delta_{4}\right)<0.00455 \quad \text { whenever } \quad \phi_{1} \leq 0.087205 \quad(l=2),
$$

and that one may take

$$
\tau_{8, l}=\frac{1}{4} \delta_{4}<0.00733 \quad \text { whenever } \quad \phi_{1} \leq 0.087205 \quad(l=2) .
$$

As in the previous cases, our computations for $s \geq 23$ depend on first obtaining preliminary estimates by applying the process $M_{6}^{s}$ for $23 \leq s \leq 29$ (noting (5.26) and checking $\left(\mathrm{D}_{1}\right)$ or $\left(\mathrm{D}_{2}\right)$ ), and $D^{s}$ for $s \geq 30$. In this way we obtain the preliminary permissible exponents

$$
\begin{gathered}
\lambda_{23}=35.299, \quad \lambda_{24}=37.244, \quad \lambda_{25}=39.199, \quad \lambda_{26}=41.161, \\
\lambda_{27}=43.130, \quad \lambda_{28}=45.105, \quad \lambda_{29}=47.084,
\end{gathered}
$$

and by virtue of the preliminary exponent

$$
\sigma(11)=0.00930 \quad(s=25, t=7, w=15),
$$

we have also

$$
\lambda_{s}=\max \{2 s-11,47.084+2(s-29)(1-0.00930)\}
$$

for $s>29$. Equipped with these preliminary bounds, we refine our procedure as indicated in the table. One may computationally check the validity of the appropriate case of Lemma 5.7 as follows.

(a) $s=23,24$. With process $N_{8}^{s}$, one finds that Lemma 5.7( $\left.\mathrm{I}^{\prime}\right)$ holds with $u=s-9$, by virtue of condition $\left(\mathrm{A}_{1}\right)$.

(b) $25 \leq s \leq 28$. With process $M_{7}^{s}$, one finds that Lemma 5.7(I) holds with $u=s-8$, by virtue of conditions $\left(\mathrm{D}_{1}\right),\left(\mathrm{C}_{1}\right),\left(\mathrm{C}_{2}\right),\left(\mathrm{C}_{3}\right)$.

(c) $s=29$. With process $N_{6}^{s}$, one finds that Lemma 5.7( $\left.\mathrm{I}^{\prime}\right)$ holds with $u=s-7$, by virtue of condition $\left(\mathrm{A}_{1}\right)$.

(d) $s \geq 30$. One finds that process $D^{s}$ applies.

14. Permissible exponents for twelfth powers. Following the computational procedure outlined in Section 8, we obtain the permissible exponents recorded in the table below. The exponents $\lambda_{s}$ listed in the table improve on those previously available for $s \geq 3$. We follow a similar path to that taken in previous sections. Note first that for $3 \leq s \leq 14$, the condition $(\delta)$ of Theorem 3.4 is satisfied. Since one of the conditions (i) and (iii) of Theorem 3.4 is also satisfied in this range of $s$, we deduce that one may take $\tau_{j, l}=0(l=1,2)$ for $3 \leq s \leq 14$. When $j=6$ and $s=15,16$, meanwhile, we must resort to Theorem 3.4(II)(1). Here we note that condition (iii) is satisfied, and thus the estimate (4.1) holds for $j=6$ and $l=2,3$ with $\chi_{j, l}=1 / 3$ and $\tau_{j, l}=1$. Next, when $j=7$ and $s=17,18$, we may apply case $(\beta)$ of Theorem 3.4(Ib) in combination with the condition (iii) to deduce that one may take $\tau_{7,2}=0$. 
Table of permissible exponents for $k=12$

\begin{tabular}{|c|c|c|c|c|c|c|}
\hline$s$ & Process & $\lambda_{s}$ & $\phi_{1}$ & $\phi_{j}$ & $\sum_{i=1}^{j} \phi_{i}$ & $\phi_{s}^{*}$ \\
\hline 3 & $B_{1,4}^{3,2}$ & 3.0173811 & 0.00869053 & & & \\
\hline 4 & $B_{1,5}^{4,2}$ & 4.1139674 & 0.03238304 & & & \\
\hline 5 & $A_{1}^{5,2}$ & 5.2806367 & 0.04288933 & & & \\
\hline 6 & $B_{2,7}^{6,2}$ & 6.5078011 & 0.04813455 & 0.0252 & 0.0733 & \\
\hline 7 & $A_{2}^{7,2}$ & 7.7910496 & 0.05157287 & 0.0392 & 0.0907 & \\
\hline 8 & $B_{3,8}^{8,2}$ & 9.1322748 & 0.05495699 & 0.0221 & 0.1252 & 0.9671 \\
\hline 9 & $A_{3}^{9,2}$ & 10.5253191 & 0.05723065 & 0.0371 & 0.1466 & 1.2185 \\
\hline 10 & $B_{4,9}^{10,2}$ & 11.9729266 & 0.05988316 & 0.0222 & 0.1871 & 1.4243 \\
\hline 11 & $A_{4}^{11,2}$ & 13.4700805 & 0.06193465 & 0.0373 & 0.2112 & 1.5174 \\
\hline 12 & $B_{5,10}^{12,2}$ & 15.0174771 & 0.06417372 & 0.0238 & 0.2566 & 1.6127 \\
\hline 13 & $A_{5}^{13,2}$ & 16.6110110 & 0.06607653 & 0.0378 & 0.2819 & 1.6761 \\
\hline 14 & $A_{5}^{14,2}$ & 18.2496682 & 0.06802194 & 0.0503 & 0.3060 & 1.7421 \\
\hline 15 & $A_{6}^{15,2}$ & 19.9296021 & 0.06973444 & 0.0387 & 0.3580 & 1.7957 \\
\hline 16 & $B_{6,13}^{16,3}$ & 21.6486622 & 0.07140335 & 0.0515 & 0.3844 & 1.8541 \\
\hline 17 & $A_{7}^{17,2}$ & 23.4028589 & 0.07285983 & 0.0378 & 0.4355 & 1.9056 \\
\hline 18 & $A_{7}^{18,2}$ & 25.1895563 & 0.07423677 & 0.0490 & 0.4606 & 1.9583 \\
\hline 19 & $A_{8}^{19,2}$ & 27.0053277 & 0.07546142 & 0.0357 & 0.5142 & 2.0143 \\
\hline 20 & $A_{8}^{20,2}$ & 28.8470137 & 0.07655400 & 0.0453 & 0.5376 & 2.0630 \\
\hline 21 & $B_{8,17}^{21,3}$ & 30.7117485 & 0.07753392 & 0.0540 & 0.5596 & 2.1187 \\
\hline 22 & $A_{9}^{22,2}$ & 32.5965148 & 0.07837940 & 0.0396 & 0.6128 & 2.1644 \\
\hline 23 & $A_{9}^{23,2}$ & 34.4988383 & 0.07912700 & 0.0477 & 0.6340 & 2.2080 \\
\hline 24 & $A_{9}^{24,2}$ & 36.4163328 & 0.07977408 & 0.0540 & 0.6516 & 2.2565 \\
\hline 25 & $A_{9}^{25,2}$ & 38.3468951 & 0.08033400 & 0.0596 & 0.6676 & 2.3037 \\
\hline 26 & $N_{9}^{26}$ & 40.2885464 & 0.08080691 & 0.0625 & 0.6782 & 2.3393 \\
\hline 27 & $N_{9}^{27}$ & 42.2395410 & 0.08120210 & 0.0625 & 0.6830 & 2.3609 \\
\hline 28 & $M_{8}^{28}$ & 44.1986746 & 0.08155581 & 0.0721 & 0.6272 & 2.3983 \\
\hline 29 & $M_{8}^{29}$ & 46.1643984 & 0.08183181 & 0.0721 & 0.6300 & 2.4054 \\
\hline 30 & $M_{8}^{30}$ & 48.1357634 & 0.08207146 & 0.0721 & 0.6323 & 2.4160 \\
\hline 31 & $M_{8}^{31}$ & 50.1118679 & 0.08227285 & 0.0721 & 0.6343 & 2.4251 \\
\hline 32 & $M_{8}^{32}$ & 52.0919461 & 0.08244173 & 0.0721 & 0.6360 & 2.4327 \\
\hline$s \geq 33$ & $D^{s}$ & & & & & \\
\hline \multicolumn{7}{|c|}{$\begin{array}{c}\sigma(12)=0.008349 \quad(s=28, t=8, w=17) \\
\tau(12)=0.010475 \quad(s=11), \quad G(12) \leq 76 \quad(v=32)\end{array}$} \\
\hline
\end{tabular}

In order to discuss permissible exponents for $19 \leq s \leq 25$, we apply case (Ic) of Theorem 3.4. In the notation of the latter theorem, we find from 
Section 23 that when $j=8$ or 9 and $10 \leq j+l \leq 11$, one has $J=1$, and hence one may take

$$
\begin{gathered}
\delta_{4}=0.0234059, \quad \delta_{5}=0.0866022, \quad \delta_{8}=0.4689321, \\
\delta_{9}=0.6501924, \quad \delta_{10}=0.8586937 .
\end{gathered}
$$

Thus we deduce that one may take

$$
\tau_{8, l}=\frac{1}{4} \delta_{4}<0.00586 \quad \text { whenever } \quad \phi_{1} \leq 0.080502 \quad(l=2,3),
$$

and that one may take

$$
\tau_{9, l}=\frac{1}{9}\left(\delta_{4}+\delta_{5}\right)<0.01223 \quad \text { whenever } \quad \phi_{1} \leq 0.078831 \quad(l=2),
$$

and otherwise, one may take

$$
\tau_{9, l}=\frac{1}{9} \delta_{9}<0.07225 \quad(l=2) .
$$

As in the previous cases, our computations for $s \geq 26$ depend on first obtaining preliminary estimates by applying the process $M_{7}^{s}$ for $26 \leq s \leq 33$ (noting (5.26) and checking $\left(\mathrm{D}_{1}\right)$ or $\left(\mathrm{D}_{2}\right)$ ), and $D^{s}$ for $s \geq 34$. In this way we obtain the preliminary permissible exponents

$$
\begin{array}{llll}
\lambda_{26}=40.290, & \lambda_{27}=42.241, & \lambda_{28}=44.200, & \lambda_{29}=46.166, \\
\lambda_{30}=48.138, & \lambda_{31}=50.114, & \lambda_{32}=52.094, & \lambda_{33}=54.077,
\end{array}
$$

and by virtue of the preliminary exponent

$$
\sigma(12)=0.00834 \quad(s=28, t=8, w=17),
$$

we have also

$$
\lambda_{s}=\max \{2 s-12,54.077+2(s-33)(1-0.00834)\}
$$

for $s>33$. Equipped with these preliminary bounds, we refine our procedure as indicated in the table. One may computationally check the validity of the appropriate case of Lemma 5.7 as follows.

(a) $s=26,27$. With process $N_{9}^{s}$, one finds that Lemma 5.7( $\left.\mathrm{I}^{\prime}\right)$ holds with $u=s-10$, by virtue of condition $\left(\mathrm{A}_{1}\right)$.

(b) $28 \leq s \leq 32$. With process $M_{8}^{s}$, one finds that Lemma 5.7(I) holds with $u=s-9$, by virtue of conditions $\left(\mathrm{D}_{1}\right),\left(\mathrm{C}_{1}\right),\left(\mathrm{C}_{2}\right),\left(\mathrm{C}_{3}\right)$.

(c) $s \geq 33$. One finds that process $D^{s}$ applies.

15. Permissible exponents for thirteenth powers. Following the computational procedure outlined in Section 8, we obtain the permissible exponents recorded in the table below. The exponents $\lambda_{s}$ listed in the table improve on those previously available for $s \geq 3$. We follow a similar path to that taken in previous sections. Note first that for $3 \leq s \leq 15$, the condition $(\delta)$ of Theorem 3.4 is satisfied. Since one of the conditions (i) and (iii) of Theorem 3.4 is also satisfied in this range of $s$, we deduce that 
one may take $\tau_{j, l}=0(l=1,2)$ for $3 \leq s \leq 15$. When $j=6$ or 7 and $s=16,17,18$, meanwhile, we must resort to Theorem 3.4(II)(1). Here we note that condition (iii) is satisfied, and thus the estimate (4.1) holds for $j=6$ and $l=2,3$, and likewise for $j=7$ and $l=2$ with $\chi_{j, l}=1 / 3$ and $\tau_{j, l}=1$. Next, when $j=8$ and $s=19,20$, we may apply case $(\beta)$ of Theorem $3.4(\mathrm{Ib})$ in combination with the condition (iii) to deduce that one may take $\tau_{8,2}=0$.

In order to discuss permissible exponents for $21 \leq s \leq 28$, we apply case (Ic) of Theorem 3.4. In the notation of the latter theorem, we find from Section 23 that when $j=9$ or 10 and $11 \leq j+l \leq 12$, one has $J=1$, and hence one may take

$$
\delta_{4}=0.0190100, \quad \delta_{5}=0.0738636, \quad \delta_{8}=0.4222375, \quad \delta_{10}=0.7792591 .
$$

Thus we deduce that one may take

$$
\tau_{9, l}=\frac{1}{9}\left(\delta_{4}+\delta_{5}\right)<0.01032 \quad \text { whenever } \quad \phi_{1} \leq 0.073360 \quad(l=2,3),
$$

and that one may take

$$
\tau_{10, l}=\frac{1}{5} \delta_{5}<0.01478 \quad \text { whenever } \quad \phi_{1} \leq 0.073360 \quad(l=2),
$$

and otherwise, one may take

$$
\tau_{10, l}=\frac{1}{10} \delta_{10}<0.07793 \quad(l=2) .
$$

As in the previous cases, our computations for $s \geq 29$ depend on first obtaining preliminary estimates by applying the process $M_{8}^{s}$ for $29 \leq s \leq 36$ (noting (5.26) and checking $\left(\mathrm{D}_{1}\right)$ or $\left(\mathrm{D}_{2}\right)$ ), and $D^{s}$ for $s \geq 37$. In this way we obtain the preliminary permissible exponents

$$
\begin{array}{llll}
\lambda_{29}=45.284, & \lambda_{30}=47.240, & \lambda_{31}=49.203, & \lambda_{32}=51.171, \\
\lambda_{33}=53.144, & \lambda_{34}=55.122, & \lambda_{35}=57.102, & \lambda_{36}=59.086,
\end{array}
$$

and by virtue of the preliminary exponent

$$
\sigma(13)=0.00755 \quad(s=31, t=9, w=18),
$$

we have also

$$
\lambda_{s}=\max \{2 s-13,59.086+2(s-36)(1-0.00755)\}
$$

for $s>36$. Equipped with these preliminary bounds, we refine our procedure as indicated in the table. One may computationally check the validity of the appropriate case of Lemma 5.7 as follows.

(a) $s=29,30$. With process $N_{10}^{s}$, one finds that Lemma 5.7( $\left.\mathrm{I}^{\prime}\right)$ holds with $u=s-11$, by virtue of condition $\left(\mathrm{A}_{1}\right)$.

(b) $31 \leq s \leq 35$. With process $M_{9}^{s}$, one finds that Lemma 5.7(I) holds with $u=s-10$, by virtue of conditions $\left(\mathrm{D}_{1}\right),\left(\mathrm{C}_{1}\right),\left(\mathrm{C}_{2}\right),\left(\mathrm{C}_{3}\right)$. 
Table of permissible exponents for $k=13$

\begin{tabular}{|c|c|c|c|c|c|c|}
\hline$s$ & Process & $\lambda_{s}$ & $\phi_{1}$ & $\phi_{j}$ & $\sum_{i=1}^{j} \phi_{i}$ & $\phi_{s}^{*}$ \\
\hline 3 & $B_{1,4}^{3,2}$ & 3.0139128 & 0.00695637 & & & \\
\hline 4 & $B_{1,5}^{4,2}$ & 4.0980713 & 0.02818356 & & & \\
\hline 5 & $A_{1}^{5,2}$ & 5.2480334 & 0.03843282 & & & \\
\hline 6 & $B_{2,7}^{6,2}$ & 6.4550068 & 0.04355532 & 0.0200 & 0.0636 & \\
\hline 7 & $B_{2,7}^{7,2}$ & 7.7136017 & 0.04663575 & 0.0344 & 0.0811 & \\
\hline 8 & $B_{3,9}^{8,2}$ & 9.0257224 & 0.04965016 & 0.0166 & 0.1095 & 0.8876 \\
\hline 9 & $A_{3}^{9,2}$ & 10.3855801 & 0.05159785 & 0.0320 & 0.1307 & 1.1586 \\
\hline 10 & $A_{3}^{10,2}$ & 11.7953083 & 0.05380951 & 0.0428 & 0.1471 & 1.3592 \\
\hline 11 & $A_{4}^{11,2}$ & 13.2521623 & 0.05568206 & 0.0317 & 0.1880 & 1.4644 \\
\hline 12 & $A_{4}^{12,2}$ & 14.7557170 & 0.05756333 & 0.0429 & 0.2072 & 1.5517 \\
\hline 13 & $A_{5}^{13,2}$ & 16.3039366 & 0.05930365 & 0.0319 & 0.2510 & 1.6178 \\
\hline 14 & $A_{5}^{14,2}$ & 17.8953488 & 0.06099508 & 0.0433 & 0.2724 & 1.6782 \\
\hline 15 & $A_{6}^{15,2}$ & 19.5276970 & 0.06257992 & 0.0324 & 0.3188 & 1.7316 \\
\hline 16 & $B_{6,13}^{16,3}$ & 21.1988910 & 0.06409230 & 0.0444 & 0.3427 & 1.7823 \\
\hline 17 & $A_{7}^{17,2}$ & 22.9062626 & 0.06549065 & 0.0345 & 0.3928 & 1.8359 \\
\hline 18 & $A_{7}^{18,2}$ & 24.6473038 & 0.06679816 & 0.0460 & 0.4176 & 1.8877 \\
\hline 19 & $A_{8}^{19,2}$ & 26.4190897 & 0.06798261 & 0.0323 & 0.4638 & 1.9380 \\
\hline 20 & $A_{8}^{20,2}$ & 28.2190701 & 0.06907751 & 0.0421 & 0.4868 & 1.9866 \\
\hline 21 & $A_{9}^{21,2}$ & 30.0445818 & 0.07007187 & 0.0302 & 0.5371 & 2.0395 \\
\hline 22 & $A_{9}^{22,2}$ & 31.8929000 & 0.07095680 & 0.0390 & 0.5591 & 2.0860 \\
\hline 23 & $B_{9,18}^{23,3}$ & 33.7616279 & 0.07175359 & 0.0470 & 0.5797 & 2.1319 \\
\hline 24 & $A_{10}^{24,2}$ & 35.6483904 & 0.07245756 & 0.0336 & 0.6289 & 2.1808 \\
\hline 25 & $A_{10}^{25,2}$ & 37.5510006 & 0.07307633 & 0.0401 & 0.6470 & 2.2212 \\
\hline 26 & $A_{10}^{26,2}$ & 39.4675269 & 0.07362249 & 0.0470 & 0.6654 & 2.2610 \\
\hline 27 & $A_{10}^{27,2}$ & 41.3961543 & 0.07409771 & 0.0521 & 0.6805 & 2.3067 \\
\hline 28 & $A_{10}^{28,2}$ & 43.3352806 & 0.07451110 & 0.0566 & 0.6939 & 2.3485 \\
\hline 29 & $N_{10}^{29}$ & 45.2834077 & 0.07486365 & 0.0577 & 0.7010 & 2.3754 \\
\hline 30 & $N_{10}^{30}$ & 47.2392765 & 0.07516706 & 0.0577 & 0.7053 & 2.3948 \\
\hline 31 & $M_{9}^{31}$ & 49.2018815 & 0.07543499 & 0.0666 & 0.6538 & 2.4262 \\
\hline 32 & $M_{9}^{32}$ & 51.1701090 & 0.07565390 & 0.0666 & 0.6565 & 2.4365 \\
\hline 33 & $M_{9}^{33}$ & 53.1431803 & 0.07584409 & 0.0666 & 0.6588 & 2.4467 \\
\hline 34 & $M_{9}^{34}$ & 55.1203776 & 0.07600614 & 0.0666 & 0.6607 & 2.4555 \\
\hline
\end{tabular}


Table of permissible exponents for $k=13$ (cont.)

\begin{tabular}{ccccccc}
\hline$s$ & Process & $\lambda_{s}$ & $\phi_{1}$ & $\phi_{j}$ & $\sum_{i=1}^{j} \phi_{i}$ & $\phi_{s}^{*}$ \\
\hline 35 & $M_{9}^{35}$ & 57.1010835 & 0.07614400 & 0.0666 & 0.6624 & 2.4629 \\
36 & $N_{8}^{36}$ & 59.0849135 & 0.07627230 & 0.0718 & 0.5990 & 2.4879 \\
$s \geq 37$ & $D^{s}$ & & & \\
\hline \multicolumn{6}{c}{$\sigma(13)=0.007556$} & $(s=31, t=9, w=18)$, \\
& $\tau(13)=0.009545$ & $(s=12)$, & $G(13) \leq 84$ & $(v=36)$ \\
\hline
\end{tabular}

(c) $s=36$. With process $N_{8}^{s}$, one finds that Lemma 5.7( $\left.\mathrm{I}^{\prime}\right)$ holds with $u=s-9$, by virtue of condition $\left(\mathrm{A}_{1}\right)$.

(d) $s \geq 37$. One finds that process $D^{s}$ applies.

16. Permissible exponents for fourteenth powers. Following the computational procedure outlined in Section 8, we obtain the permissible exponents recorded in the table below. The exponents $\lambda_{s}$ listed in the table improve on those previously available for $s \geq 3$. We follow a similar path to that taken in previous sections. Note first that for $3 \leq s \leq 16$, the condition ( $\delta$ ) of Theorem 3.4 is satisfied. Since one of the conditions (i) and (iii) of Theorem 3.4 is also satisfied in this range of $s$, we deduce that one may take $\tau_{j, l}=0(l=1,2)$ for $3 \leq s \leq 16$. When $j=7$ or 8 and $17 \leq s \leq 20$, meanwhile, we must resort to Theorem 3.4(II)(1). Here we note that condition (iii) is satisfied, and thus the estimate (4.1) holds for $j=7,8$ with $\chi_{j, 2}=1 / 3$ and $\tau_{j, 2}=1$. Next, when $j=9$ and $s=21$, we may apply case $(\beta)$ of Theorem $3.4(\mathrm{Ib})$ in combination with the condition (iii) to deduce that one may take $\tau_{9,2}=0$.

In order to discuss permissible exponents for $22 \leq s \leq 30$, we apply case (Ic) of Theorem 3.4. In the notation of the latter theorem, we find from Section 23 that when $j=9$ and $l=2$, one has $J=2$, and hence one may take

$$
\delta_{4}=0.0018248, \quad \delta_{5}=0.0107720, \quad \delta_{8}=0.1314920, \quad \delta_{10}=0.2835673,
$$

whence we may take

$$
\tau_{9, l}=\frac{1}{9}\left(\delta_{4}+\delta_{5}\right)<0.00140 \quad \text { whenever } \quad \phi_{1} \leq 0.070116 \quad(l=2) .
$$

Also from Section 23, we find that when $j=9,10,11$ and $12 \leq j+l \leq 13$, one has $J=1$, and hence one may take

$$
\begin{aligned}
& \delta_{4}=0.0156211, \quad \delta_{5}=0.0633584, \quad \delta_{6}=0.1434849, \\
& \delta_{8}=0.3829073, \quad \delta_{10}=0.7106189, \quad \delta_{11}=0.9079284 .
\end{aligned}
$$

Thus we deduce that one may take

$$
\tau_{9, l}=\frac{1}{9}\left(\delta_{4}+\delta_{5}\right)<0.00878 \quad \text { whenever } \quad \phi_{1} \leq 0.068568 \quad(l=2,3),
$$


Table of permissible exponents for $k=14$

\begin{tabular}{|c|c|c|c|c|c|c|}
\hline$s$ & Process & $\lambda_{s}$ & $\phi_{1}$ & $\phi_{j}$ & $\sum_{i=1}^{j} \phi_{i}$ & $\phi_{s}^{*}$ \\
\hline 3 & $B_{1,4}^{3,2}$ & 3.0113494 & 0.00567466 & & & \\
\hline 4 & $B_{1,5}^{4,2}$ & 4.0856057 & 0.02484611 & & & \\
\hline 5 & $A_{1}^{5,2}$ & 5.2216967 & 0.03476683 & & & \\
\hline 6 & $B_{2,7}^{6,2}$ & 6.4118310 & 0.03979118 & 0.0160 & 0.0557 & \\
\hline 7 & $B_{2,7}^{7,2}$ & 7.6492691 & 0.04248943 & 0.0303 & 0.0728 & \\
\hline 8 & $A_{2}^{8,2}$ & 8.9350975 & 0.04500717 & 0.0383 & 0.0834 & \\
\hline 9 & $A_{3}^{9,2}$ & 10.2671180 & 0.04699577 & 0.0277 & 0.1175 & 1.1041 \\
\hline 10 & $A_{3}^{10,2}$ & 11.6442024 & 0.04876376 & 0.0373 & 0.1317 & 1.2919 \\
\hline 11 & $B_{4,10}^{11,2}$ & 13.0662900 & 0.05051435 & 0.0268 & 0.1686 & 1.4194 \\
\hline 12 & $A_{4}^{12,2}$ & 14.5316795 & 0.05209364 & 0.0370 & 0.1855 & 1.4979 \\
\hline 13 & $B_{5,11}^{13,2}$ & 16.0399382 & 0.05367993 & 0.0268 & 0.2251 & 1.5676 \\
\hline 14 & $A_{5}^{14,2}$ & 17.5892784 & 0.05515430 & 0.0373 & 0.2442 & 1.6228 \\
\hline 15 & $A_{6}^{15,2}$ & 19.1785686 & 0.05660418 & 0.0273 & 0.2865 & 1.6759 \\
\hline 16 & $A_{6}^{16,2}$ & 20.8058286 & 0.05796461 & 0.0383 & 0.3079 & 1.7234 \\
\hline 17 & $A_{7}^{17,2}$ & 22.4692736 & 0.05926701 & 0.0287 & 0.3527 & 1.7725 \\
\hline 18 & $A_{7}^{18,2}$ & 24.1667258 & 0.06048642 & 0.0398 & 0.3756 & 1.8209 \\
\hline 19 & $A_{8}^{19,2}$ & 25.8960050 & 0.06162954 & 0.0306 & 0.4230 & 1.8711 \\
\hline 20 & $A_{8}^{20,2}$ & 27.6547883 & 0.06268866 & 0.0409 & 0.4458 & 1.9192 \\
\hline 21 & $A_{9}^{21,2}$ & 29.4407200 & 0.06366289 & 0.0276 & 0.4884 & 1.9661 \\
\hline 22 & $A_{9}^{22,2}$ & 31.2515093 & 0.06455699 & 0.0367 & 0.5102 & 2.0125 \\
\hline 23 & $B_{9,18}^{23,3}$ & 33.0849211 & 0.06537338 & 0.0449 & 0.5306 & 2.0596 \\
\hline 24 & $A_{10}^{24,2}$ & 34.9387022 & 0.06610731 & 0.0337 & 0.5776 & 2.1056 \\
\hline 25 & $A_{10}^{25,2}$ & 36.8107871 & 0.06676863 & 0.0408 & 0.5967 & 2.1481 \\
\hline 26 & $B_{10,20}^{26,3}$ & 38.6992193 & 0.06736052 & 0.0469 & 0.6137 & 2.1932 \\
\hline 27 & $A_{11}^{27,2}$ & 40.6021475 & 0.06788535 & 0.0357 & 0.6616 & 2.2350 \\
\hline 28 & $A_{11}^{28,2}$ & 42.5178927 & 0.06835016 & 0.0410 & 0.6774 & 2.2719 \\
\hline 29 & $A_{11}^{29,2}$ & 44.4449274 & 0.06876037 & 0.0458 & 0.6916 & 2.3080 \\
\hline 30 & $A_{11}^{30,2}$ & 46.3818646 & 0.06912079 & 0.0500 & 0.7046 & 2.3493 \\
\hline 31 & $N_{11}^{31}$ & 48.3274553 & 0.06943614 & 0.0536 & 0.7161 & 2.3867 \\
\hline 32 & $N_{11}^{32}$ & 50.2805449 & 0.06970829 & 0.0536 & 0.7206 & 2.4066 \\
\hline 33 & $N_{11}^{33}$ & 52.2401670 & 0.06994609 & 0.0536 & 0.7245 & 2.4242 \\
\hline 34 & $N_{10}^{34}$ & 54.2054937 & 0.07015541 & 0.0619 & 0.6767 & 2.4527 \\
\hline 35 & $N_{10}^{35}$ & 56.1756866 & 0.07033184 & 0.0619 & 0.6793 & 2.4637 \\
\hline 36 & $N_{10}^{36}$ & 58.1501035 & 0.07048574 & 0.0619 & 0.6815 & 2.4733 \\
\hline 37 & $N_{10}^{37}$ & 60.1281620 & 0.07061847 & 0.0619 & 0.6834 & 2.4817 \\
\hline
\end{tabular}


Table of permissible exponents for $k=14$ (cont.)

\begin{tabular}{|c|c|c|c|c|c|c|}
\hline$s$ & Process & $\lambda_{s}$ & $\phi_{1}$ & $\phi_{j}$ & $\sum_{i=1}^{j} \phi_{i}$ & $\phi_{s}^{*}$ \\
\hline 38 & $M_{10}^{38}$ & 62.1093556 & 0.07073278 & 0.0619 & 0.6851 & 2.4889 \\
\hline 39 & $N_{9}^{39}$ & 64.0933213 & 0.07083658 & 0.0667 & 0.6264 & 2.5149 \\
\hline 40 & $N_{9}^{40}$ & 66.0795485 & 0.07091753 & 0.0667 & 0.6274 & 2.5175 \\
\hline$s \geq 41$ & $D^{s}$ & & & & & \\
\hline & $\tau(14) \stackrel{\sigma(1}{=}$ & $\begin{array}{l})=0.006895 \\
0.008770\end{array}$ & $\begin{aligned} & (s=34, t= \\
= & 13), \quad G(1\end{aligned}$ & $\begin{array}{l}10, w= \\
\leq 92\end{array}$ & $\begin{array}{l}19), \\
(v=40)\end{array}$ & \\
\hline
\end{tabular}

that one may take

$$
\tau_{10, l}=\frac{1}{5} \delta_{5}<0.01268 \quad \text { whenever } \quad \phi_{1} \leq 0.068568 \quad(l=2,3),
$$

and that one may take

$$
\tau_{11, l}=\frac{1}{11} \delta_{11}<0.08254 \quad(l=2) .
$$

As in the previous cases, our computations for $s \geq 31$ depend on first obtaining preliminary estimates by applying the process $M_{9}^{s}$ for $31 \leq s \leq 40$ (noting (5.26) and checking $\left(\mathrm{D}_{1}\right)$ or $\left(\mathrm{D}_{2}\right)$ ), and $D^{s}$ for $s \geq 41$. In this way we obtain the preliminary permissible exponents

$\lambda_{31}=48.328, \quad \lambda_{32}=50.281, \quad \lambda_{33}=52.241, \quad \lambda_{34}=54.206, \quad \lambda_{35}=56.177$,

$\lambda_{36}=58.151, \quad \lambda_{37}=60.129, \quad \lambda_{38}=62.110, \quad \lambda_{39}=64.094, \quad \lambda_{40}=66.080$,

and by virtue of the preliminary exponent

$$
\sigma(14)=0.00689 \quad(s=34, t=10, w=19),
$$

we have also

$$
\lambda_{s}=\max \{2 s-14,66.080+2(s-40)(1-0.00689)\}
$$

for $s>40$. Equipped with these preliminary bounds, we refine our procedure as indicated in the table. One may computationally check the validity of the appropriate case of Lemma 5.7 as follows.

(a) $31 \leq s \leq 33$. With process $N_{11}^{s}$, one finds that Lemma $5.7\left(\mathrm{I}^{\prime}\right)$ holds with $u=s-12$, by virtue of condition $\left(\mathrm{A}_{1}\right)$.

(b) $34 \leq s \leq 37$. With process $N_{10}^{s}$, one finds that Lemma $5.7\left(\mathrm{I}^{\prime}\right)$ holds with $u=s-11$, by virtue of condition $\left(\mathrm{A}_{1}\right)$.

(c) $s=38$. With process $M_{10}^{s}$, one finds that Lemma $5.7(\mathrm{I})$ holds with $u=s-11$, by virtue of conditions $\left(\mathrm{D}_{1}\right),\left(\mathrm{C}_{1}\right),\left(\mathrm{C}_{2}\right),\left(\mathrm{C}_{3}\right)$.

(d) $s=39,40$. With process $N_{9}^{s}$, one finds that Lemma $5.7\left(\mathrm{I}^{\prime}\right)$ holds with $u=s-10$, by virtue of condition $\left(\mathrm{A}_{1}\right)$.

(e) $s \geq 41$. One finds that process $D^{s}$ applies. 
17. Permissible exponents for fifteenth powers. Following the computational procedure outlined in Section 8, we obtain the permissible exponents recorded in the table below. The exponents $\lambda_{s}$ listed in the table improve on those previously available for $s \geq 3$. We follow a similar path to that taken in previous sections. Note first that for $3 \leq s \leq 17$, the condition $(\delta)$ of Theorem 3.4 is satisfied. Since one of the conditions (i) and (iii) of Theorem 3.4 is also satisfied in this range of $s$, we deduce that one may take $\tau_{j, l}=0(l=1,2)$ for $3 \leq s \leq 17$. When $j=7,8,9$ and $18 \leq s \leq 22$, meanwhile, we must resort to Theorem 3.4(II)(1). Here we note that condition (iii) is satisfied, and thus the estimate (4.1) holds for $j=7,8,9$ with $\chi_{j, 2}=1 / 3$ and $\tau_{j, 2}=1$. Next, when $j=10$ and $s=23$, we may apply case $(\beta)$ of Theorem 3.4(Ib) in combination with the condition (iii) to deduce that one may take $\tau_{10,2}=0$.

In order to discuss permissible exponents for $24 \leq s \leq 33$, we apply case (Ic) of Theorem 3.4. In the notation of the latter theorem, we find from Section 23 that when $j=10$ and $l=2$, one has $J=2$, and hence one may take

$$
\delta_{5}=0.0088563 \quad \text { and } \quad \delta_{10}=0.2565541,
$$

whence we may take

$$
\tau_{10, l}=\frac{1}{5} \delta_{5}<0.00178 \quad \text { whenever } \quad \phi_{1} \leq 0.065621 \quad(l=2) .
$$

Further, when $j=11,12$ and $13 \leq j+l \leq 14$, one has $J=1$, and hence one may take

$$
\delta_{5}=0.0536213, \quad \delta_{6}=0.1264298, \quad \delta_{12}=1.0354619 .
$$

Thus we deduce that one may take

$$
\tau_{11, l}=\frac{1}{11}\left(\delta_{5}+\delta_{6}\right)<0.01637 \quad \text { whenever } \quad \phi_{1} \leq 0.063360 \quad(l=2,3),
$$

and that one may take

$$
\tau_{12, l}=\frac{1}{12} \delta_{12}<0.08629 \quad(l=2) .
$$

As in the previous cases, our computations for $s \geq 34$ depend on first obtaining preliminary estimates by applying the process $M_{10}^{s}$ for $34 \leq s \leq 44$ (noting (5.26) and checking $\left(\mathrm{D}_{1}\right)$ or $\left(\mathrm{D}_{2}\right)$ ), and $D^{s}$ for $s \geq 45$. In this way we obtain the preliminary permissible exponents

$$
\begin{aligned}
& \lambda_{34}=53.323, \quad \lambda_{35}=55.280, \quad \lambda_{36}=57.243, \quad \lambda_{37}=59.210, \\
& \lambda_{38}=61.182, \quad \lambda_{39}=63.157, \quad \lambda_{40}=65.136, \quad \lambda_{41}=67.118, \\
& \lambda_{42}=69.102, \quad \lambda_{43}=71.088, \quad \lambda_{44}=73.076,
\end{aligned}
$$

and by virtue of the preliminary exponent

$$
\sigma(15)=0.00633 \quad(s=37, t=11, w=21),
$$


Table of permissible exponents for $k=15$

\begin{tabular}{|c|c|c|c|c|c|c|}
\hline$s$ & Process & $\lambda_{s}$ & $\phi_{1}$ & $\phi_{j}$ & $\sum_{i=1}^{j} \phi_{i}$ & $\phi_{s}^{*}$ \\
\hline 3 & $B_{1,4}^{3,2}$ & 3.0092359 & 0.00461792 & & & \\
\hline 4 & $B_{1,6}^{4,2}$ & 4.0742295 & 0.02173146 & & & \\
\hline 5 & $A_{1}^{5,2}$ & 5.1987873 & 0.03172823 & & & \\
\hline 6 & $B_{2,8}^{6,2}$ & 6.3738989 & 0.03647237 & 0.0116 & 0.0481 & \\
\hline 7 & $B_{2,7}^{7,2}$ & 7.5936427 & 0.03905794 & 0.0269 & 0.0659 & \\
\hline 8 & $A_{2}^{8,2}$ & 8.8571841 & 0.04113748 & 0.0342 & 0.0753 & \\
\hline 9 & $B_{3,9}^{9,2}$ & 10.1649995 & 0.04309441 & 0.0236 & 0.1058 & 1.0461 \\
\hline 10 & $A_{3}^{10,2}$ & 11.5141981 & 0.04456907 & 0.0327 & 0.1189 & 1.2336 \\
\hline 11 & $B_{4,10}^{11,2}$ & 12.9059619 & 0.04616698 & 0.0222 & 0.1517 & 1.3792 \\
\hline 12 & $A_{4}^{12,2}$ & 14.3381411 & 0.04752336 & 0.0321 & 0.1674 & 1.4522 \\
\hline 13 & $B_{5,11}^{13,2}$ & 15.8111209 & 0.04895330 & 0.0223 & 0.2032 & 1.5241 \\
\hline 14 & $A_{5}^{14,2}$ & 17.3230870 & 0.05024754 & 0.0321 & 0.2205 & 1.5749 \\
\hline 15 & $A_{6}^{15,2}$ & 18.8736139 & 0.05156238 & 0.0229 & 0.2592 & 1.6271 \\
\hline 16 & $A_{6}^{16,2}$ & 20.4609219 & 0.05278515 & 0.0331 & 0.2784 & 1.6717 \\
\hline 17 & $A_{7}^{17,2}$ & 22.0838491 & 0.05398415 & 0.0239 & 0.3190 & 1.7169 \\
\hline 18 & $A_{7}^{18,2}$ & 23.7405373 & 0.05510909 & 0.0340 & 0.3394 & 1.7623 \\
\hline 19 & $A_{8}^{19,2}$ & 25.4293639 & 0.05618735 & 0.0257 & 0.3830 & 1.8090 \\
\hline 20 & $A_{8}^{20,2}$ & 27.1483206 & 0.05719334 & 0.0356 & 0.4044 & 1.8549 \\
\hline 21 & $A_{9}^{21,2}$ & 28.8955107 & 0.05813950 & 0.0271 & 0.4492 & 1.9020 \\
\hline 22 & $A_{9}^{22,2}$ & 30.6688816 & 0.05901573 & 0.0367 & 0.4710 & 1.9480 \\
\hline 23 & $A_{10}^{23,2}$ & 32.4664265 & 0.05982581 & 0.0239 & 0.5104 & 1.9912 \\
\hline 24 & $A_{10}^{24,2}$ & 34.2861492 & 0.06056957 & 0.0320 & 0.5305 & 2.0351 \\
\hline 25 & $A_{10}^{25,2}$ & 36.1261310 & 0.06125062 & 0.0397 & 0.5499 & 2.0782 \\
\hline 26 & $A_{11}^{26,2}$ & 37.9844896 & 0.06186873 & 0.0290 & 0.5938 & 2.1226 \\
\hline 27 & $A_{11}^{27,2}$ & 39.8594364 & 0.06242704 & 0.0358 & 0.6119 & 2.1634 \\
\hline 28 & $B_{11,22}^{28,3}$ & 41.7493055 & 0.06293025 & 0.0417 & 0.6284 & 2.2023 \\
\hline 29 & $A_{12}^{29,2}$ & 43.6525286 & 0.06338099 & 0.0310 & 0.6730 & 2.2453 \\
\hline 30 & $A_{12}^{30,2}$ & 45.5676489 & 0.06378269 & 0.0359 & 0.6878 & 2.2808 \\
\hline 31 & $A_{12}^{31,2}$ & 47.4933457 & 0.06414041 & 0.0405 & 0.7015 & 2.3144 \\
\hline 32 & $A_{12}^{32,2}$ & 49.4284091 & 0.06445755 & 0.0444 & 0.7139 & 2.3488 \\
\hline 33 & $A_{12}^{33,2}$ & 51.3717431 & 0.06473789 & 0.0479 & 0.7251 & 2.3859 \\
\hline 34 & $N_{12}^{34}$ & 53.3223547 & 0.06498462 & 0.0500 & 0.7335 & 2.4158 \\
\hline 35 & $N_{12}^{35}$ & 55.2793490 & 0.06520081 & 0.0500 & 0.7376 & 2.4338 \\
\hline 36 & $N_{11}^{36}$ & 57.2419678 & 0.06539241 & 0.0578 & 0.6939 & 2.4654 \\
\hline 37 & $N_{11}^{37}$ & 59.2094705 & 0.06555771 & 0.0578 & 0.6966 & 2.4771 \\
\hline
\end{tabular}


Table of permissible exponents for $k=15$ (cont.)

\begin{tabular}{|c|c|c|c|c|c|c|}
\hline$s$ & Process & $\lambda_{s}$ & $\phi_{1}$ & $\phi_{j}$ & $\sum_{i=1}^{j} \phi_{i}$ & $\phi_{s}^{*}$ \\
\hline 38 & $N_{11}^{38}$ & 61.1812515 & 0.06570293 & 0.0578 & 0.6991 & 2.4875 \\
\hline 39 & $N_{11}^{39}$ & 63.1567645 & 0.06582965 & 0.0578 & 0.7012 & 2.4965 \\
\hline 40 & $N_{11}^{40}$ & 65.1355287 & 0.06594009 & 0.0578 & 0.7031 & 2.5045 \\
\hline 41 & $M_{11}^{41}$ & 67.1171222 & 0.06603623 & 0.0578 & 0.7047 & 2.5115 \\
\hline 42 & $N_{10}^{42}$ & 69.1012148 & 0.06612247 & 0.0623 & 0.6500 & 2.5383 \\
\hline 43 & $N_{10}^{43}$ & 71.0874163 & 0.06619342 & 0.0623 & 0.6511 & 2.5409 \\
\hline$s \geq 44$ & $D^{s}$ & & & & & \\
\hline \multicolumn{7}{|c|}{$\begin{array}{c}\sigma(15)=0.006338 \quad(s=37, t=11, w=21) \\
\tau(15)=0.008114 \quad(s=14), \quad G(15) \leq 100 \quad(v=43)\end{array}$} \\
\hline
\end{tabular}

we have also

$$
\lambda_{s}=\max \{2 s-15,73.076+2(s-44)(1-0.00633)\}
$$

for $s>44$. Equipped with these preliminary bounds, we refine our procedure as indicated in the table. One may computationally check the validity of the appropriate case of Lemma 5.7 as follows.

(a) $s=34,35$. With process $N_{12}^{s}$, one finds that Lemma 5.7( $\left.\mathrm{I}^{\prime}\right)$ holds with $u=s-13$, by virtue of condition $\left(\mathrm{A}_{1}\right)$.

(b) $36 \leq s \leq 40$. With process $N_{11}^{s}$, one finds that Lemma $5.7\left(\mathrm{I}^{\prime}\right)$ holds with $u=s-12$, by virtue of condition $\left(\mathrm{A}_{1}\right)$.

(c) $s=41$. With process $M_{11}^{s}$, one finds that Lemma 5.7(I) holds with $u=s-12$, by virtue of conditions $\left(\mathrm{D}_{1}\right),\left(\mathrm{C}_{1}\right),\left(\mathrm{C}_{2}\right),\left(\mathrm{C}_{3}\right)$.

(d) $s=42,43$. With process $N_{10}^{s}$, one finds that Lemma 5.7( $\left.\mathrm{I}^{\prime}\right)$ holds with $u=s-11$, by virtue of condition $\left(\mathrm{A}_{1}\right)$.

(e) $s \geq 44$. One finds that process $D^{s}$ applies.

18. Permissible exponents for sixteenth powers. Following the computational procedure outlined in Section 8, we obtain the permissible exponents recorded in the table below. The exponents $\lambda_{s}$ listed in the table improve on those previously available for $s \geq 3$. We follow a similar path to that taken in previous sections. Note first that for $3 \leq s \leq 18$, the condition ( $\delta$ ) of Theorem 3.4 is satisfied. Since one of the conditions (i) and (iii) of Theorem 3.4 is also satisfied in this range of $s$, we deduce that one may take $\tau_{j, l}=0(l=1,2)$ for $3 \leq s \leq 18$. When $j=8,9,10$ and $19 \leq s \leq 24$, meanwhile, we must resort to Theorem 3.4(II)(1). Here we note that condition (iii) is satisfied, and thus the estimate (4.1) holds for $j=8,9,10$ with $\chi_{j, 2}=1 / 3$ and $\tau_{j, 2}=1$.

In order to discuss permissible exponents for $25 \leq s \leq 36$, we apply case (Ic) of Theorem 3.4. In the notation of the latter theorem, we find from 
Section 23 that when $j=11$ and $l=1$ or 2 , one has $J=2$, and hence one may take

$$
\delta_{5}=0.0073658, \quad \delta_{6}=0.0241263, \quad \delta_{12}=0.4026992,
$$

whence we may take

$$
\tau_{11, l}=\frac{1}{11}\left(\delta_{5}+\delta_{6}\right)<0.00287 \quad \text { whenever } \quad \phi_{1} \leq 0.061146 \quad(l=1,2) .
$$

Further, when $j=11,12,13$ and $14 \leq j+l \leq 15$, one has $J=1$, and hence one may take

$$
\delta_{5}=0.0460456, \quad \delta_{6}=0.1127790, \quad \delta_{12}=0.9585035, \quad \delta_{13}=1.1659645 .
$$

Thus we deduce that one may take

$$
\tau_{11, l}=\frac{1}{11}\left(\delta_{5}+\delta_{6}\right)<0.01444 \quad \text { whenever } \quad \phi_{1} \leq 0.059762 \quad(l=3),
$$

that one may take

$$
\tau_{12, l}=\frac{1}{6} \delta_{6}<0.01880 \quad \text { whenever } \quad \phi_{1} \leq 0.059762 \quad(l=2),
$$

and that one may take

$$
\tau_{13, l}=\frac{1}{13} \delta_{13}<0.08969 \quad(l=2) .
$$

As in the previous cases, our computations for $s \geq 37$ depend on first obtaining preliminary estimates by applying the process $M_{10}^{s}$ for $37 \leq s \leq 47$ (noting (5.27) and checking $\left(\mathrm{D}_{1}\right)$ or $\left(\mathrm{D}_{2}\right)$ ), and $D^{s}$ for $s \geq 48$. In this way we obtain the preliminary permissible exponents

$$
\begin{array}{llll}
\lambda_{37}=58.320, & \lambda_{38}=60.280, & \lambda_{39}=62.245, & \lambda_{40}=64.215, \\
\lambda_{41}=66.188, & \lambda_{42}=68.164, & \lambda_{43}=70.143, & \lambda_{44}=72.125, \\
\lambda_{45}=74.110, & \lambda_{46}=76.096, & \lambda_{47}=78.084,
\end{array}
$$

and by virtue of the preliminary exponent

$$
\sigma(16)=0.00586 \quad(s=41, t=11, w=23),
$$

we have also

$$
\lambda_{s}=\max \{2 s-16,78.084+2(s-47)(1-0.00586)\}
$$

for $s>47$. Equipped with these preliminary bounds, we refine our procedure as indicated in the table. One may computationally check the validity of the appropriate case of Lemma 5.7 as follows.

(a) $s=37,38$. With process $N_{13}^{s}$, one finds that Lemma 5.7( $\left.\mathrm{I}^{\prime}\right)$ holds with $u=s-14$, by virtue of condition $\left(\mathrm{A}_{1}\right)$.

(b) $39 \leq s \leq 43$. With process $N_{12}^{s}$, one finds that Lemma $5.7\left(\mathrm{I}^{\prime}\right)$ holds with $u=s-13$, by virtue of condition $\left(\mathrm{A}_{1}\right)$.

(c) $s=44$. With process $M_{12}^{s}$, one finds that Lemma 5.7(I) holds with $u=s-13$, by virtue of conditions $\left(\mathrm{D}_{1}\right),\left(\mathrm{C}_{1}\right),\left(\mathrm{C}_{2}\right),\left(\mathrm{C}_{3}\right)$. 
Table of permissible exponents for $k=16$

\begin{tabular}{|c|c|c|c|c|c|c|}
\hline$s$ & Process & $\lambda_{s}$ & $\phi_{1}$ & $\phi_{j}$ & $\sum_{i=1}^{j} \phi_{i}$ & $\phi_{s}^{*}$ \\
\hline 3 & $B_{1,4}^{3,2}$ & 3.0074816 & 0.00374079 & & & \\
\hline 4 & $B_{1,6}^{4,2}$ & 4.0638321 & 0.01883046 & & & \\
\hline 5 & $A_{1}^{5,2}$ & 5.1787385 & 0.02919246 & & & \\
\hline 6 & $B_{2,8}^{6,2}$ & 6.3412246 & 0.03370200 & 0.0083 & 0.0420 & \\
\hline 7 & $B_{2,8}^{7,2}$ & 7.5457229 & 0.03613827 & 0.0241 & 0.0602 & \\
\hline 8 & $A_{2}^{8,2}$ & 8.7902441 & 0.03788514 & 0.0308 & 0.0687 & \\
\hline 9 & $B_{3,9}^{9,2}$ & 10.0769155 & 0.03976160 & 0.0200 & 0.0957 & 0.9893 \\
\hline 10 & $A_{3}^{10,2}$ & 11.4019639 & 0.04102548 & 0.0289 & 0.1082 & 1.1825 \\
\hline 11 & $B_{4,10}^{11,2}$ & 12.7671799 & 0.04247669 & 0.0185 & 0.1375 & 1.3449 \\
\hline 12 & $A_{4}^{12,2}$ & 14.1702162 & 0.04365257 & 0.0280 & 0.1522 & 1.4130 \\
\hline 13 & $B_{5,12}^{13,2}$ & 15.6119303 & 0.04493631 & 0.0182 & 0.1842 & 1.4861 \\
\hline 14 & $A_{5}^{14,2}$ & 17.0906280 & 0.04608149 & 0.0276 & 0.2003 & 1.5333 \\
\hline 15 & $B_{6,13}^{15,2}$ & 18.6062852 & 0.04726736 & 0.0188 & 0.2354 & 1.5841 \\
\hline 16 & $A_{6}^{16,2}$ & 20.1573791 & 0.04836825 & 0.0285 & 0.2531 & 1.6259 \\
\hline 17 & $B_{7,14}^{17,2}$ & 21.7431712 & 0.04946474 & 0.0198 & 0.2902 & 1.6685 \\
\hline 18 & $A_{7}^{18,2}$ & 23.3621148 & 0.05049786 & 0.0293 & 0.3090 & 1.7107 \\
\hline 19 & $A_{8}^{19,2}$ & 25.0129891 & 0.05150184 & 0.0211 & 0.3485 & 1.7539 \\
\hline 20 & $A_{8}^{20,2}$ & 26.6941169 & 0.05244686 & 0.0306 & 0.3682 & 1.7972 \\
\hline 21 & $A_{9}^{21,2}$ & 28.4039654 & 0.05334847 & 0.0229 & 0.4098 & 1.8413 \\
\hline 22 & $A_{9}^{22,2}$ & 30.1407641 & 0.05419218 & 0.0322 & 0.4304 & 1.8854 \\
\hline 23 & $A_{10}^{23,2}$ & 31.9028113 & 0.05498480 & 0.0243 & 0.4729 & 1.9297 \\
\hline 24 & $A_{10}^{24,2}$ & 33.6883143 & 0.05572055 & 0.0329 & 0.4931 & 1.9734 \\
\hline 25 & $B_{11,19}^{25,2}$ & 35.4955330 & 0.05640278 & 0.0205 & 0.5297 & 2.0143 \\
\hline 26 & $A_{11}^{26,2}$ & 37.3227332 & 0.05703073 & 0.0279 & 0.5485 & 2.0548 \\
\hline 27 & $A_{11}^{27,2}$ & 39.1682523 & 0.05760740 & 0.0351 & 0.5668 & 2.0961 \\
\hline 28 & $B_{11,21}^{28,3}$ & 41.0304795 & 0.05813390 & 0.0408 & 0.5828 & 2.1367 \\
\hline 29 & $A_{12}^{29,2}$ & 42.9078717 & 0.05861191 & 0.0313 & 0.6252 & 2.1765 \\
\hline 30 & $A_{12}^{30,2}$ & 44.7989875 & 0.05904508 & 0.0369 & 0.6410 & 2.2140 \\
\hline 31 & $B_{12,24}^{31,3}$ & 46.7024729 & 0.05943588 & 0.0414 & 0.6547 & 2.2506 \\
\hline 32 & $A_{13}^{32,2}$ & 48.6170673 & 0.05978708 & 0.0316 & 0.6971 & 2.2884 \\
\hline 33 & $A_{13}^{33,2}$ & 50.5416092 & 0.06010180 & 0.0358 & 0.7101 & 2.3210 \\
\hline 34 & $A_{13}^{34,2}$ & 52.4750359 & 0.06038318 & 0.0396 & 0.7220 & 2.3515 \\
\hline 35 & $A_{13}^{35,2}$ & 54.4163771 & 0.06063404 & 0.0429 & 0.7329 & 2.3839 \\
\hline 36 & $A_{13}^{36,2}$ & 56.3647518 & 0.06085714 & 0.0459 & 0.7428 & 2.4179 \\
\hline 37 & $N_{13}^{37}$ & 58.3193587 & 0.06105480 & 0.0469 & 0.7488 & 2.4413 \\
\hline
\end{tabular}


Table of permissible exponents for $k=16$ (cont.)

\begin{tabular}{|c|c|c|c|c|c|c|}
\hline$s$ & Process & $\lambda_{s}$ & $\phi_{1}$ & $\phi_{j}$ & $\sum_{i=1}^{j} \phi_{i}$ & $\phi_{s}^{*}$ \\
\hline 38 & $N_{13}^{38}$ & 60.2794807 & 0.06122977 & 0.0469 & 0.7526 & 2.4577 \\
\hline 39 & $N_{12}^{39}$ & 62.2444892 & 0.06138528 & 0.0543 & 0.7115 & 2.4878 \\
\hline 40 & $N_{12}^{40}$ & 64.2137908 & 0.06152144 & 0.0543 & 0.7141 & 2.4987 \\
\hline 41 & $N_{12}^{41}$ & 66.1868812 & 0.06164181 & 0.0543 & 0.7164 & 2.5084 \\
\hline 42 & $N_{12}^{42}$ & 68.1633061 & 0.06174778 & 0.0543 & 0.7185 & 2.5169 \\
\hline 43 & $N_{12}^{43}$ & 70.1426626 & 0.06184097 & 0.0543 & 0.7203 & 2.5246 \\
\hline 44 & $M_{12}^{44}$ & 72.1245941 & 0.06192285 & 0.0543 & 0.7219 & 2.5313 \\
\hline 45 & $N_{11}^{45}$ & 74.1088058 & 0.06199601 & 0.0584 & 0.6708 & 2.5587 \\
\hline 46 & $N_{11}^{46}$ & 76.0949856 & 0.06205826 & 0.0584 & 0.6718 & 2.5613 \\
\hline 47 & $N_{11}^{47}$ & 78.0829008 & 0.06211345 & 0.0584 & 0.6727 & 2.5636 \\
\hline$s \geq 48$ & \multicolumn{6}{|l|}{$D^{s}$} \\
\hline \multicolumn{7}{|c|}{$\begin{array}{c}\sigma(16)=0.005864 \quad(s=41, t=11, w=23) \\
\tau(16)=0.007549 \quad(s=15), \quad G(16) \leq 109 \quad(v=47)\end{array}$} \\
\hline
\end{tabular}

(d) $45 \leq s \leq 47$. With process $N_{11}^{s}$, one finds that Lemma $5.7\left(\mathrm{I}^{\prime}\right)$ holds with $u=s-12$, by virtue of condition $\left(\mathrm{A}_{1}\right)$.

(e) $s \geq 48$. One finds that process $D^{s}$ applies.

19. Permissible exponents for seventeenth powers. Following the computational procedure outlined in Section 8, we obtain the permissible exponents recorded in the table below. The exponents $\lambda_{s}$ listed in the table improve on those previously available for $s \geq 3$. We follow a similar path to that taken in previous sections. Note first that for $3 \leq s \leq 19$, the condition $(\delta)$ of Theorem 3.4 is satisfied. Since one of the conditions (i) and (iii) of Theorem 3.4 is also satisfied in this range of $s$, we deduce that one may take $\tau_{j, l}=0(l=1,2)$ for $3 \leq s \leq 19$. When $8 \leq j \leq 11$ and $20 \leq s \leq 27$, meanwhile, we must resort to Theorem 3.4(II)(1) and (2). Here we note that condition (iii) is satisfied for $20 \leq s \leq 26$, and that (II)(2) applies for $s=27$, and thus the estimate (4.1) holds for $8 \leq j \leq 11$ and $l=2,3$ with $\chi_{j, l}=1 / 3$ and $\tau_{j, l}=1$. Note here that when $s=27$, the relevant value of $\sigma$ is so small that the condition $\phi_{1}+\ldots+\phi_{j} \geq \frac{1}{3}(1-\sigma)^{-1}$ is satisfied transparently.

In order to discuss permissible exponents for $28 \leq s \leq 39$, we apply case (Ic) of Theorem 3.4. In the notation of the latter theorem, we find from Section 23 that when $j=12$ and $l=2$, one has $J=2$, and hence one may take

$$
\delta_{6}=0.0205621 \text { and } \delta_{12}=0.3706630,
$$

whence we may take

$$
\tau_{12, l}=\frac{1}{6} \delta_{6}<0.00343 \quad \text { whenever } \quad \phi_{1} \leq 0.057704 \quad(l=2) .
$$


Table of permissible exponents for $k=17$

\begin{tabular}{|c|c|c|c|c|c|c|}
\hline$s$ & Process & $\lambda_{s}$ & $\phi_{1}$ & $\phi_{j}$ & $\sum_{i=1}^{j} \phi_{i}$ & $\phi_{s}^{*}$ \\
\hline 3 & $B_{1,4}^{3,2}$ & 3.0061404 & 0.00307019 & & & \\
\hline 4 & $B_{1,6}^{4,2}$ & 4.0554335 & 0.01646473 & & & \\
\hline 5 & $A_{1}^{5,2}$ & 5.1619828 & 0.02701166 & & & \\
\hline 6 & $B_{2,8}^{6,2}$ & 6.3136071 & 0.03134018 & 0.0056 & 0.0369 & \\
\hline 7 & $B_{2,8}^{7,2}$ & 7.5040992 & 0.03349964 & 0.0210 & 0.0545 & \\
\hline 8 & $A_{2}^{8,2}$ & 8.7323022 & 0.03513032 & 0.0278 & 0.0630 & \\
\hline 9 & $B_{3,10}^{9,2}$ & 10.0004288 & 0.03689292 & 0.0168 & 0.0869 & 0.9360 \\
\hline 0 & $A_{3}^{10,2}$ & 11.3043577 & 0.03799316 & 0.0257 & 0.0991 & 1.1377 \\
\hline 1 & $A_{3}^{11,2}$ & 12.6459140 & 0.03927903 & 0.0330 & 0.1097 & 1.3053 \\
\hline & $A_{4}^{12,2}$ & 14.0233197 & 0.04034662 & 0.0245 & 0.1392 & 1.3790 \\
\hline 3 & $A_{4}^{13,2}$ & 15.4369512 & 0.04145984 & 0.0321 & 0.1516 & 1.4456 \\
\hline 4 & $A_{5}^{14,2}$ & 16.8860867 & 0.04251950 & 0.0239 & 0.1831 & 1.4968 \\
\hline 5 & $A_{5}^{15,2}$ & 18.3702735 & 0.04356583 & 0.0325 & 0.1980 & 1.5438 \\
\hline 6 & $A_{6}^{16,2}$ & 19.8886761 & 0.04457565 & 0.0244 & 0.2314 & 1.5855 \\
\hline 7 & $A_{6}^{17,2}$ & 21.4405373 & 0.04556572 & 0.0332 & 0.2477 & 1.6256 \\
\hline 8 & $A_{7}^{18,2}$ & 23.0247863 & 0.04651864 & & 0.2827 & 1.6648 \\
\hline 9 & $A_{7}^{19,2}$ & 24.6403737 & 0.04744334 & 0.0339 & 0.3001 & 1.7059 \\
\hline 0 & $A_{8}^{20,2}$ & 26.2860071 & 0.04832721 & 0.0259 & 0.3366 & 1.7458 \\
\hline 21 & $B_{8,17}^{21,3}$ & 27.9604002 & 0.04917555 & & 0.3554 & 1.7878 \\
\hline 2 & $A_{9}^{22,2}$ & 29.6620874 & 0.04997915 & 0.0279 & 0.3945 & 1.8289 \\
\hline 3 & $B_{9,18}^{23,3}$ & 31.3896134 & 0.05074142 & 0.0362 & 0.4131 & 1.8710 \\
\hline 24 & $A_{10}^{24,2}$ & 33.1414245 & 0.05145731 & 0.0291 & 0.4534 & 1.9126 \\
\hline 25 & $B_{10,19}^{25,3}$ & 34.9159903 & 0.05212921 & 0.0368 & 0.4717 & 1.9544 \\
\hline 6 & $A_{11}^{26,2}$ & 36.7117441 & 0.05275480 & & 0.5126 & 1.9959 \\
\hline 7 & $B_{11,20}^{27,3}$ & 38.5271601 & 0.05333611 & 0.0367 & 0.5307 & 2.0372 \\
\hline 8 & $A_{12}^{28,2}$ & 40.3607211 & 0.05387253 & 0.0245 & 0.5647 & 2.0725 \\
\hline 29 & $A_{12}^{29,2}$ & 42.2109803 & 0.05436691 & 0.0310 & 0.5818 & 2.1119 \\
\hline 30 & $B_{12,23}^{30,3}$ & 44.0765377 & 0.05482022 & 0.0367 & 0.5976 & 2.1499 \\
\hline 1 & $A_{13}^{31,2}$ & 45.9560566 & 0.05523415 & 0.0275 & 0.6371 & 2.1879 \\
\hline 2 & $A_{13}^{32,2}$ & 47.8482757 & 0.05561097 & 0.0328 & 0.6521 & 2.2240 \\
\hline 33 & $B_{13,25}^{33,3}$ & 49.7520144 & 0.05595308 & 0.0379 & 0.6667 & 2.2594 \\
\hline 34 & $B_{13,25}^{34,3}$ & 51.6661665 & 0.05626250 & 0.0409 & 0.6775 & 2.2946 \\
\hline 35 & $A_{14}^{35,2}$ & 53.5897087 & 0.05654168 & 0.0318 & 0.7177 & 2.3262 \\
\hline 36 & $A_{14}^{36,2}$ & 55.5216990 & 0.05679304 & 0.0354 & 0.7292 & 2.3562 \\
\hline 7 & $A_{14}^{37,2}$ & 57.4612719 & 0.05701880 & 0.0386 & 0.7398 & 2.3843 \\
\hline
\end{tabular}


Table of permissible exponents for $k=17$ (cont.)

\begin{tabular}{|c|c|c|c|c|c|c|}
\hline$s$ & Process & $\lambda_{s}$ & $\phi_{1}$ & $\phi_{j}$ & $\sum_{i=1}^{j} \phi_{i}$ & $\phi_{s}^{*}$ \\
\hline 38 & $A_{14}^{38,2}$ & 59.4076368 & 0.05722114 & 0.0414 & 0.7493 & 2.4146 \\
\hline 39 & $A_{14}^{39,2}$ & 61.3600741 & 0.05740216 & 0.0439 & 0.7581 & 2.4458 \\
\hline 40 & $N_{14}^{40}$ & 63.3179278 & 0.05756359 & 0.0442 & 0.7624 & 2.4638 \\
\hline 41 & $N_{14}^{41}$ & 65.2806096 & 0.05770757 & 0.0442 & 0.7658 & 2.4789 \\
\hline 42 & $N_{13}^{42}$ & 67.2475940 & 0.05783611 & 0.0511 & 0.7271 & 2.5076 \\
\hline 43 & $N_{13}^{43}$ & 69.2183942 & 0.05794990 & 0.0511 & 0.7296 & 2.5178 \\
\hline 44 & $N_{13}^{44}$ & 71.1925852 & 0.05805112 & 0.0511 & 0.7318 & 2.5269 \\
\hline 45 & $N_{13}^{45}$ & 73.1697840 & 0.05814094 & 0.0511 & 0.7337 & 2.5350 \\
\hline 46 & $N_{13}^{46}$ & 75.1496484 & 0.05822056 & 0.0511 & 0.7355 & 2.5423 \\
\hline 47 & $N_{12}^{47}$ & 77.1318848 & 0.05829175 & 0.0550 & 0.6878 & 2.5738 \\
\hline 48 & $N_{12}^{48}$ & 79.1162028 & 0.05835376 & 0.0550 & 0.6890 & 2.5767 \\
\hline 49 & $N_{12}^{49}$ & 81.1023673 & 0.05840894 & 0.0550 & 0.6901 & 2.5793 \\
\hline 50 & $N_{12}^{50}$ & 83.0901643 & 0.05845772 & 0.0550 & 0.6910 & 2.5816 \\
\hline$s \geq 51$ & \multicolumn{6}{|l|}{$D^{s}$} \\
\hline \multicolumn{7}{|c|}{$\begin{array}{c}\sigma(17)=0.005454 \quad(s=44, t=12, w=24) \\
\tau(17)=0.007060 \quad(s=16), \quad G(17) \leq 117 \quad(v=50)\end{array}$} \\
\hline
\end{tabular}

Further, when $j=12,13,14$ and $15 \leq j+l \leq 16$, one has $J=1$, and hence one may take

$$
\begin{gathered}
\delta_{6}=0.1004200, \quad \delta_{7}=0.1840767, \quad \delta_{12}=0.8905163, \\
\delta_{13}=1.0847742, \quad \delta_{14}=1.2966345 .
\end{gathered}
$$

Thus we deduce that one may take

$$
\tau_{12, l}=\frac{1}{6} \delta_{6}<0.01674 \quad \text { whenever } \quad \phi_{1} \leq 0.056530 \quad(l=3),
$$

that one may take

$$
\tau_{13, l}=\frac{1}{13}\left(\delta_{6}+\delta_{7}\right)<0.02189 \quad \text { whenever } \quad \phi_{1} \leq 0.055777 \quad(l=2),
$$

and otherwise,

$$
\tau_{13, l}=\frac{1}{13} \delta_{13}<0.08345 \quad(l=2,3),
$$

and that one may take

$$
\tau_{14, l}=\frac{1}{14} \delta_{14}<0.09262 \quad(l=2) .
$$

As in the previous cases, our computations for $s \geq 40$ depend on first obtaining preliminary estimates by applying the process $M_{11}^{s}$ for $40 \leq s \leq 51$ (noting (5.27) and checking $\left(\mathrm{D}_{1}\right)$ or $\left(\mathrm{D}_{2}\right)$ ), and $D^{s}$ for $s \geq 52$. In this way 
we obtain the preliminary permissible exponents

$$
\begin{array}{llll}
\lambda_{40}=63.318, & \lambda_{41}=65.281, & \lambda_{42}=67.248, & \lambda_{43}=69.219, \\
\lambda_{44}=71.193, & \lambda_{45}=73.170, & \lambda_{46}=75.150, & \lambda_{47}=77.133, \\
\lambda_{48}=79.117, & \lambda_{49}=81.103, & \lambda_{50}=83.091, & \lambda_{51}=85.080,
\end{array}
$$

and by virtue of the preliminary exponent

$$
\sigma(17)=0.00545 \quad(s=44, t=12, w=24),
$$

we have also

$$
\lambda_{s}=\max \{2 s-17,85.080+2(s-51)(1-0.00545)\}
$$

for $s>51$. Equipped with these preliminary bounds, we refine our procedure as indicated in the table. One may computationally check the validity of the appropriate case of Lemma 5.7 as follows.

(a) $s=40,41$. With process $N_{14}^{s}$, one finds that Lemma 5.7( $\left.\mathrm{I}^{\prime}\right)$ holds with $u=s-15$, by virtue of condition $\left(\mathrm{A}_{1}\right)$.

(b) $42 \leq s \leq 46$. With process $N_{13}^{s}$, one finds that Lemma $5.7\left(\mathrm{I}^{\prime}\right)$ holds with $u=s-14$, by virtue of condition $\left(\mathrm{A}_{1}\right)$.

(c) $47 \leq s \leq 50$. With process $N_{12}^{s}$, one finds that Lemma 5.7( $\left.\mathrm{I}^{\prime}\right)$ holds with $u=s-13$, by virtue of condition $\left(\mathrm{A}_{1}\right)$.

(d) $s \geq 51$. One finds that process $D^{s}$ applies.

20. Permissible exponents for eighteenth powers. Following the computational procedure outlined in Section 8, we obtain the permissible exponents recorded in the table below. The exponents $\lambda_{s}$ listed in the table improve on those previously available for $s \geq 3$. We follow a similar path to that taken in previous sections. Note first that for $3 \leq s \leq 21$, the condition $(\delta)$ of Theorem 3.4 is satisfied. Since one of the conditions (i) and (iii) of Theorem 3.4 is also satisfied in this range of $s$, we deduce that one may take $\tau_{j, l}=0(l=1,2,3)$ for $3 \leq s \leq 21$. When $9 \leq j \leq 12$ and $22 \leq s \leq 29$, meanwhile, we must resort to Theorem $3.4(\mathrm{II})(1)$ and (2). Here we note that condition (iii) is satisfied for $22 \leq s \leq 27$, and that (II)(2) applies for $s=28,29$, and thus the estimate (4.1) holds for $9 \leq j \leq 12$ and $l=2,3$ with $\chi_{j, l}=1 / 3$ and $\tau_{j, l}=1$. Note here that when $s=28,29$, the relevant value of $\sigma$ is so small that the condition $\phi_{1}+\ldots+\phi_{j} \geq \frac{1}{3}(1-\sigma)^{-1}$ is satisfied transparently.

In order to discuss permissible exponents for $30 \leq s \leq 41$, we apply case (Ic) of Theorem 3.4. In the notation of the latter theorem, we find from Section 23 that when $j=13$ and $l=2$, one has $J=2$, and hence one may take

$$
\delta_{6}=0.0176775, \quad \delta_{7}=0.0427686, \quad \delta_{14}=0.5236334,
$$


whence we may take

$$
\tau_{13, l}=\frac{1}{13}\left(\delta_{6}+\delta_{7}\right)<0.00465 \quad \text { whenever } \quad \phi_{1} \leq 0.054235 \quad(l=2) .
$$

Further, when $j=13,14,15$ and $16 \leq j+l \leq 17$, one has $J=1$, and hence one may take

$$
\delta_{6}=0.0901459, \quad \delta_{7}=0.1679365, \quad \delta_{14}=1.2130601, \quad \delta_{15}=1.4286845 .
$$

Thus we deduce that one may take

$$
\tau_{13, l}=\frac{1}{13}\left(\delta_{6}+\delta_{7}\right)<0.01986 \quad \text { whenever } \quad \phi_{1} \leq 0.052973 \quad(l=3),
$$

that one may take

$$
\tau_{14, l}=\frac{1}{7} \delta_{7}<0.02400 \quad \text { whenever } \quad \phi_{1} \leq 0.052973 \quad(l=2,3),
$$

and otherwise,

$$
\tau_{14, l}=\frac{1}{14} \delta_{14}<0.08665 \quad(l=2,3),
$$

and that one may take

$$
\tau_{15, l}=\frac{1}{15} \delta_{15}<0.09525 \quad(l=2) .
$$

As in the previous cases, our computations for $s \geq 42$ depend on first obtaining preliminary estimates by applying the process $M_{12}^{s}$ for $42 \leq s \leq 54$ (noting (5.27) and checking $\left(\mathrm{D}_{1}\right)$ or $\left(\mathrm{D}_{2}\right)$ ), and $D^{s}$ for $s \geq 55$. In this way we obtain the preliminary permissible exponents

$$
\begin{aligned}
& \lambda_{42}=66.358, \quad \lambda_{43}=68.318, \quad \lambda_{44}=70.283, \quad \lambda_{45}=72.252, \quad \lambda_{46}=74.224, \\
& \lambda_{47}=76.199, \quad \lambda_{48}=78.177, \quad \lambda_{49}=80.157, \quad \lambda_{50}=82.140, \quad \lambda_{51}=84.124 \text {, } \\
& \lambda_{52}=86.110, \quad \lambda_{53}=88.098, \quad \lambda_{54}=90.087 \text {, }
\end{aligned}
$$

and by virtue of the preliminary exponent

$$
\sigma(18)=0.00509 \quad(s=47, t=13, w=26),
$$

we have also

$$
\lambda_{s}=\max \{2 s-18,90.087+2(s-54)(1-0.00509)\}
$$

for $s>54$. Equipped with these preliminary bounds, we refine our procedure as indicated in the table. One may computationally check the validity of the appropriate case of Lemma 5.7 as follows.

(a) $42 \leq s \leq 44$. With process $N_{15}^{s}$, one finds that Lemma 5.7( $\left.\mathrm{I}^{\prime}\right)$ holds with $u=s-16$, by virtue of condition $\left(\mathrm{A}_{1}\right)$.

(b) $45 \leq s \leq 49$. With process $N_{14}^{s}$, one finds that Lemma $5.7\left(\mathrm{I}^{\prime}\right)$ holds with $u=s-15$, by virtue of condition $\left(\mathrm{A}_{1}\right)$.

(c) $50 \leq s \leq 54$. With process $N_{13}^{s}$, one finds that Lemma 5.7( $\left(\mathrm{I}^{\prime}\right)$ holds with $u=s-14$, by virtue of condition $\left(\mathrm{A}_{1}\right)$.

(d) $s \geq 55$. One finds that process $D^{s}$ applies. 
Table of permissible exponents for $k=18$

\begin{tabular}{|c|c|c|c|c|c|c|}
\hline$s$ & Process & $\lambda_{s}$ & $\phi_{1}$ & $\phi_{j}$ & $\sum_{i=1}^{j} \phi_{i}$ & $\phi_{s}^{*}$ \\
\hline 3 & $B_{1,4}^{3,2}$ & 3.0050870 & 0.00254346 & & & \\
\hline 4 & $B_{1,6}^{4,2}$ & 4.0484489 & 0.01447855 & & & \\
\hline 5 & $B_{1,6}^{5,2}$ & 5.1469671 & 0.02493153 & & & \\
\hline 6 & $B_{2,8}^{6,2}$ & 6.2893077 & 0.02933022 & 0.0034 & 0.0328 & \\
\hline 7 & $B_{2,8}^{7,2}$ & 7.4676627 & 0.03123178 & 0.0185 & 0.0497 & \\
\hline 8 & $A_{2}^{8,2}$ & 8.6816032 & 0.03275099 & 0.0254 & 0.0581 & \\
\hline 9 & $B_{3,10}^{9,2}$ & 9.9332381 & 0.03438389 & 0.0135 & 0.0787 & 0.8757 \\
\hline 10 & $A_{3}^{10,2}$ & 11.2185989 & 0.03537489 & 0.0230 & 0.0913 & 1.0979 \\
\hline 11 & $A_{3}^{11,2}$ & 12.5388988 & 0.03647482 & 0.0295 & 0.1006 & 1.2547 \\
\hline 12 & $B_{4,11}^{12,2}$ & 13.8936352 & 0.03749420 & 0.0213 & 0.1278 & 1.3486 \\
\hline 13 & $A_{4}^{13,2}$ & 15.2823305 & 0.03846045 & 0.0284 & 0.1390 & 1.4100 \\
\hline 14 & $A_{5}^{14,2}$ & 16.7049932 & 0.03943607 & 0.0206 & 0.1683 & 1.4650 \\
\hline 15 & $A_{5}^{15,2}$ & 18.1609021 & 0.04036376 & 0.0285 & 0.1816 & 1.5081 \\
\hline 16 & $A_{6}^{16,2}$ & 19.6497248 & 0.04128885 & 0.0209 & 0.2126 & 1.5495 \\
\hline 17 & $A_{6}^{17,2}$ & 21.1706906 & 0.04218253 & 0.0290 & 0.2273 & 1.5872 \\
\hline 18 & $A_{7}^{18,2}$ & 22.7231221 & 0.04306012 & 0.0215 & 0.2599 & 1.6240 \\
\hline 19 & $A_{7}^{19,2}$ & 24.3060925 & 0.04390870 & 0.0296 & 0.2757 & 1.6626 \\
\hline 20 & $A_{8}^{20,2}$ & 25.9186447 & 0.04473174 & 0.0222 & 0.3097 & 1.7001 \\
\hline 21 & $B_{8,17}^{21,3}$ & 27.5596769 & 0.04552348 & 0.0306 & 0.3268 & 1.7397 \\
\hline 22 & $A_{9}^{22,2}$ & 29.2280189 & 0.04628304 & 0.0237 & 0.3628 & 1.7781 \\
\hline 23 & $B_{9,18}^{23,3}$ & 30.9224199 & 0.04700798 & 0.0321 & 0.3807 & 1.8185 \\
\hline 24 & $A_{10}^{24,2}$ & 32.6415685 & 0.04769656 & 0.0253 & 0.4180 & 1.8574 \\
\hline 25 & $B_{10,19}^{25,3}$ & 34.3841221 & 0.04834828 & 0.0331 & 0.4359 & 1.8979 \\
\hline 26 & $A_{11}^{26,2}$ & 36.1487034 & 0.04896179 & 0.0263 & 0.4739 & 1.9370 \\
\hline 27 & $B_{11,20}^{27,3}$ & 37.9339369 & 0.04953749 & 0.0337 & 0.4917 & 1.9773 \\
\hline 28 & $A_{12}^{28,2}$ & 39.7384452 & 0.05007502 & 0.0266 & 0.5304 & 2.0163 \\
\hline 29 & $B_{12,22}^{29,3}$ & 41.5608774 & 0.05057526 & 0.0334 & 0.5475 & 2.0558 \\
\hline 30 & $A_{13}^{30,2}$ & 43.3999089 & 0.05103871 & 0.0214 & 0.5792 & 2.0894 \\
\hline 31 & $A_{13}^{31,2}$ & 45.2542644 & 0.05146692 & 0.0274 & 0.5952 & 2.1257 \\
\hline 32 & $B_{13,25}^{32,3}$ & 47.1227175 & 0.05186115 & 0.0330 & 0.6106 & 2.1626 \\
\hline 33 & $B_{13,24}^{33,3}$ & 49.0040975 & 0.05222287 & 0.0371 & 0.6234 & 2.1972 \\
\hline 34 & $A_{14}^{34,2}$ & 50.8972954 & 0.05255372 & 0.0291 & 0.6620 & 2.2327 \\
\hline 35 & $A_{14}^{35,2}$ & 52.8012695 & 0.05285563 & 0.0335 & 0.6753 & 2.2662 \\
\hline 36 & $B_{14,27}^{36,3}$ & 54.7150443 & 0.05313037 & 0.0374 & 0.6874 & 2.2979 \\
\hline 37 & $A_{15}^{37,2}$ & 56.6377106 & 0.05337974 & 0.0283 & 0.7246 & 2.3305 \\
\hline
\end{tabular}


Table of permissible exponents for $k=18$ (cont.)

\begin{tabular}{ccccccc}
\hline$s$ & Process & $\lambda_{s}$ & $\phi_{1}$ & $\phi_{j}$ & $\sum_{i=1}^{j} \phi_{i}$ & $\phi_{s}^{*}$ \\
\hline 38 & $A_{15}^{38,2}$ & 58.5684269 & 0.05360563 & 0.0317 & 0.7356 & 2.3597 \\
39 & $A_{15}^{39,2}$ & 60.5064172 & 0.05380985 & 0.0347 & 0.7458 & 2.3872 \\
40 & $A_{15}^{40,2}$ & 62.4509681 & 0.05399414 & 0.0375 & 0.7552 & 2.4132 \\
41 & $A_{15}^{41,2}$ & 64.4014262 & 0.05416015 & 0.0399 & 0.7637 & 2.4418 \\
42 & $N_{15}^{42}$ & 66.3571950 & 0.05430945 & 0.0417 & 0.7709 & 2.4685 \\
43 & $N_{15}^{43}$ & 68.3177304 & 0.05444347 & 0.0417 & 0.7744 & 2.4838 \\
44 & $N_{15}^{44}$ & 70.2825406 & 0.05456372 & 0.0417 & 0.7776 & 2.4977 \\
45 & $N_{14}^{45}$ & 72.2511825 & 0.05467161 & 0.0483 & 0.7410 & 2.5253 \\
46 & $N_{14}^{46}$ & 74.2232486 & 0.05476794 & 0.0483 & 0.7433 & 2.5349 \\
47 & $N_{14}^{47}$ & 76.1983771 & 0.05485415 & 0.0483 & 0.7454 & 2.5434 \\
48 & $N_{14}^{48}$ & 78.1762412 & 0.05493118 & 0.0483 & 0.7473 & 2.5512 \\
49 & $N_{14}^{49}$ & 80.1565468 & 0.05499994 & 0.0483 & 0.7490 & 2.5581 \\
50 & $N_{13}^{50}$ & 82.1390360 & 0.05506161 & 0.0519 & 0.7040 & 2.5899 \\
51 & $N_{13}^{51}$ & 84.1234628 & 0.05511611 & 0.0519 & 0.7052 & 2.5927 \\
52 & $N_{13}^{52}$ & 86.1096189 & 0.05516484 & 0.0519 & 0.7063 & 2.5953 \\
53 & $N_{13}^{53}$ & 88.0973151 & 0.05520823 & 0.0519 & 0.7072 & 2.5975 \\
54 & $N_{13}^{54}$ & 90.0863822 & 0.05524686 & 0.0519 & 0.7081 & 2.5996 \\
$\geq 55$ & $D^{s}$ & & & & & \\
\hline & & & & & & \\
\hline
\end{tabular}

21. Permissible exponents for nineteenth powers. Following the computational procedure outlined in Section 8, we obtain the permissible exponents recorded in the table below. The exponents $\lambda_{s}$ listed in the table improve on those previously available for $s \geq 3$. We follow a similar path to that taken in previous sections. Note first that for $3 \leq s \leq 21$, the condition $(\delta)$ of Theorem 3.4 is satisfied. Since one of the conditions (i) and (iii) of Theorem 3.4 is also satisfied in this range of $s$, we deduce that one may take $\tau_{j, l}=0(l=1,2)$ for $3 \leq s \leq 21$. When $9 \leq j \leq 13$ and $22 \leq s \leq 31$, meanwhile, we must resort to Theorem $3.4($ II)(1) and (2). Here we note that condition (iii) is satisfied for $22 \leq s \leq 29$, and that (II)(2) applies for $s=30,31$, and thus the estimate (4.1) holds for $9 \leq j \leq 13$ and $l=2,3$ with $\chi_{j, l}=1 / 3$ and $\tau_{j, l}=1$. Note here that when $s=30,31$, the relevant value of $\sigma$ is so small that the condition $\phi_{1}+\ldots+\phi_{j} \geq \frac{1}{3}(1-\sigma)^{-1}$ is satisfied transparently.

In order to discuss permissible exponents for $32 \leq s \leq 44$, we apply case (Ic) of Theorem 3.4. In the notation of the latter theorem, we find from 
Table of permissible exponents for $k=19$

\begin{tabular}{|c|c|c|c|c|c|c|}
\hline$s$ & Process & $\lambda_{s}$ & $\phi_{1}$ & $\phi_{j}$ & $\sum_{i=1}^{j} \phi_{i}$ & $\phi_{s}^{*}$ \\
\hline 3 & $B_{1,4}^{3,2}$ & 3.0042273 & 0.00211363 & & & \\
\hline 4 & $B_{1,6}^{4,2}$ & 4.0423620 & 0.01272951 & & & \\
\hline 5 & $B_{1,6}^{5,2}$ & 5.1333114 & 0.02298073 & & & \\
\hline 6 & $A_{1}^{6,2}$ & 6.2663273 & 0.02733191 & & & \\
\hline 7 & $B_{2,8}^{7,2}$ & 7.4343072 & 0.02929710 & 0.0164 & 0.0457 & \\
\hline 8 & $A_{2}^{8,2}$ & 8.6357245 & 0.03067723 & 0.0233 & 0.0540 & \\
\hline 9 & $A_{2}^{9,2}$ & 9.8725415 & 0.03215755 & 0.0286 & 0.0608 & \\
\hline 0 & $B_{3,10}^{10,2}$ & 11.1414574 & 0.03308734 & 0.0205 & 0.0843 & 1.0573 \\
\hline 1 & $A_{3}^{11,2}$ & 12.4430359 & 0.03404381 & 0.0266 & 0.0929 & 1.2102 \\
\hline 2 & $B_{4,11}^{12,2}$ & 13.7775620 & 0.03500339 & 0.0186 & 0.1180 & 1.3222 \\
\hline 13 & $A_{4}^{13,2}$ & 15.1440547 & 0.03585180 & 0.0252 & 0.1282 & 1.3792 \\
\hline 4 & $A_{5}^{14,2}$ & 16.5429734 & 0.03674656 & 0.0179 & 0.1555 & 1.4369 \\
\hline 5 & $A_{5}^{15,2}$ & 17.9734687 & 0.03757479 & 0.0250 & 0.1674 & 1.4766 \\
\hline 6 & $B_{6,14}^{16,2}$ & 19.4355306 & 0.03842022 & 0.0178 & 0.1962 & 1.5175 \\
\hline 7 & $A_{6}^{17,2}$ & 20.9284296 & 0.03922960 & 0.0253 & 0.2096 & 1.5529 \\
\hline 8 & $B_{7,15}^{18,2}$ & 22.4517574 & 0.04003558 & 0.0182 & 0.2399 & 1.5885 \\
\hline 9 & $A_{7}^{19,2}$ & 24.0047167 & 0.04081410 & 0.0259 & 0.2544 & 1.6239 \\
\hline 20 & $A_{8}^{20,2}$ & 25.5866021 & 0.04157726 & 0.0189 & 0.2861 & 1.6601 \\
\hline 21 & $A_{8}^{21,2}$ & 27.1964861 & 0.04231369 & 0.0269 & 0.3020 & 1.6966 \\
\hline 22 & $A_{9}^{22,2}$ & 28.8334329 & 0.04302673 & 0.0199 & 0.3348 & 1.7332 \\
\hline 3 & $A_{9}^{23,2}$ & 30.4963841 & 0.04371136 & 0.0281 & 0.3518 & 1.7708 \\
\hline 24 & $A_{10}^{24,2}$ & 32.1842434 & 0.04436768 & 0.0216 & 0.3865 & 1.8075 \\
\hline 25 & $A_{10}^{25,2}$ & 33.8958472 & 0.04499335 & 0.0295 & 0.4037 & 1.8464 \\
\hline 26 & $A_{11}^{26,2}$ & 35.6300014 & 0.04558788 & 0.0229 & 0.4392 & 1.8832 \\
\hline 27 & $B_{11,21}^{27,3}$ & 37.3854842 & 0.04615045 & 0.0304 & 0.4565 & 1.9222 \\
\hline 28 & $A_{12}^{28,2}$ & 39.1610622 & 0.04668075 & 0.0239 & 0.4928 & 1.9592 \\
\hline 29 & $B_{12,22}^{29,3}$ & 40.9555027 & 0.04717878 & 0.0307 & 0.5096 & 1.9978 \\
\hline 30 & $A_{13}^{30,2}$ & 42.7675849 & 0.04764484 & 0.0240 & 0.5463 & 2.0346 \\
\hline 1 & $A_{13}^{31,2}$ & 44.5961098 & 0.04807945 & 0.0304 & 0.5628 & 2.0727 \\
\hline 32 & $A_{14}^{32,2}$ & 46.4399090 & 0.04848337 & 0.0186 & 0.5922 & 2.1057 \\
\hline 33 & $A_{14}^{33,2}$ & 48.2978543 & 0.04885769 & 0.0243 & 0.6075 & 2.1383 \\
\hline 34 & $A_{14}^{34,2}$ & 50.1688626 & 0.04920355 & 0.0296 & 0.6222 & 2.1738 \\
\hline ני & $B_{14,26}^{35,3}$ & 52.0518997 & 0.04952220 & 0.0338 & 0.6350 & 2.2073 \\
\hline 36 & & 53.9459843 & 0.04981500 & 0.0258 & 0.6710 & 2.2402 \\
\hline 37 & $B_{15,29}^{37,3}$ & 55.8501910 & 0.05008342 & 0.0307 & 0.6849 & 2.2740 \\
\hline
\end{tabular}


Table of permissible exponents for $k=19$ (cont.)

\begin{tabular}{|c|c|c|c|c|c|c|}
\hline$s$ & Process & $\lambda_{s}$ & $\phi_{1}$ & $\phi_{j}$ & $\sum_{i=1}^{j} \phi_{i}$ & $\phi_{s}^{*}$ \\
\hline 38 & $B_{15,29}^{38,3}$ & 57.7636509 & 0.05032891 & 0.0342 & 0.6960 & 2.3043 \\
\hline 39 & $B_{15,28}^{39,3}$ & 59.6855524 & 0.05055296 & 0.0366 & 0.7053 & 2.3329 \\
\hline 40 & $A_{15}^{40,3}$ & 61.6151397 & 0.05075705 & 0.0382 & 0.7129 & 2.3620 \\
\hline 41 & $A_{15}^{41,3}$ & 63.5517128 & 0.05094263 & 0.0397 & 0.7198 & 2.3888 \\
\hline 42 & $A_{15}^{42,3}$ & 65.4946250 & 0.05111110 & 0.0410 & 0.7262 & 2.4141 \\
\hline 43 & $A_{15}^{43,3}$ & 67.4432807 & 0.05126380 & 0.0422 & 0.7321 & 2.4379 \\
\hline 44 & $A_{15}^{44,3}$ & 69.3971334 & 0.05140202 & 0.0433 & 0.7376 & 2.4632 \\
\hline 45 & $N_{16}^{45}$ & 71.3556823 & 0.05152695 & 0.0395 & 0.7819 & 2.4876 \\
\hline 46 & $N_{16}^{46}$ & 73.3184698 & 0.05163973 & 0.0395 & 0.7852 & 2.5017 \\
\hline 47 & $N_{16}^{47}$ & 75.2850793 & 0.05174146 & 0.0395 & 0.7882 & 2.5146 \\
\hline 48 & $N_{15}^{48}$ & 77.2551336 & 0.05183320 & 0.0458 & 0.7534 & 2.5413 \\
\hline 49 & $N_{15}^{49}$ & 79.2282862 & 0.05191569 & 0.0458 & 0.7557 & 2.5502 \\
\hline 50 & $N_{15}^{50}$ & 81.2042263 & 0.05198993 & 0.0458 & 0.7577 & 2.5583 \\
\hline 51 & $N_{15}^{51}$ & 83.1826716 & 0.05205667 & 0.0458 & 0.7595 & 2.5657 \\
\hline 52 & $N_{15}^{52}$ & 85.1633672 & 0.05211663 & 0.0458 & 0.7611 & 2.5723 \\
\hline 53 & $N_{14}^{53}$ & 87.1460856 & 0.05217061 & 0.0492 & 0.7186 & 2.6042 \\
\hline 54 & $N_{14}^{54}$ & 89.1306147 & 0.05221882 & 0.0492 & 0.7197 & 2.6070 \\
\hline 55 & $N_{14}^{55}$ & 91.1167691 & 0.05226214 & 0.0492 & 0.7208 & 2.6095 \\
\hline 56 & $N_{14}^{56}$ & 93.1043803 & 0.05230098 & 0.0492 & 0.7217 & 2.6118 \\
\hline 57 & $N_{14}^{57}$ & 95.0932973 & 0.05233578 & 0.0492 & 0.7226 & 2.6138 \\
\hline 58 & $N_{14}^{58}$ & 97.0833838 & 0.05236696 & 0.0492 & 0.7234 & 2.6156 \\
\hline$s \geq 59$ & $D^{s}$ & & & & & \\
\hline \multicolumn{7}{|c|}{$\begin{array}{c}\sigma(19)=0.004780 \quad(s=50, t=13, w=28) \\
\tau(19)=0.006252 \quad(s=18), \quad G(19) \leq 134 \quad(v=58)\end{array}$} \\
\hline
\end{tabular}

Section 23 that when $j=14$ and $l=2$, one has $J=2$, and hence one may take

$$
\delta_{7}=0.0375713 \quad \text { and } \quad \delta_{14}=0.4889692,
$$

whence we may take

$$
\tau_{14, l}=\frac{1}{7} \delta_{7}<0.00537 \quad \text { whenever } \quad \phi_{1} \leq 0.051509 \quad(l=2) .
$$

Further, when $j=14,15$ and $17 \leq j+l \leq 18$, one has $J=1$, and hence one may take

$$
\begin{aligned}
\delta_{7} & =0.1541435, \quad \delta_{8}=0.2475913, \quad \delta_{14}=1.1394461, \\
\delta_{15} & =1.3428361, \quad \delta_{16}=1.5615789 .
\end{aligned}
$$


Thus we deduce that one may take

$$
\tau_{14, l}=\frac{1}{7} \delta_{7}<0.02203 \quad \text { whenever } \quad \phi_{1} \leq 0.050425 \quad(l=3),
$$

that one may take

$$
\tau_{15, l}=\frac{1}{15}\left(\delta_{7}+\delta_{8}\right)<0.02679 \quad \text { whenever } \quad \phi_{1} \leq 0.049834 \quad(l=2,3),
$$

and otherwise,

$$
\tau_{15, l}=\frac{1}{15} \delta_{15}<0.08953 \quad(l=2,3) .
$$

As in the previous cases, our computations for $s \geq 45$ depend on first obtaining preliminary estimates by applying the process $M_{13}^{s}$ for $45 \leq s \leq 58$ (noting (5.27) and checking $\left(\mathrm{D}_{1}\right)$ or $\left(\mathrm{D}_{2}\right)$ ), and $D^{s}$ for $s \geq 59$. In this way we obtain the preliminary permissible exponents

$$
\begin{aligned}
& \lambda_{45}=71.356, \quad \lambda_{46}=73.319, \quad \lambda_{47}=75.286, \quad \lambda_{48}=77.256, \quad \lambda_{49}=79.229 \text {, } \\
& \lambda_{50}=81.205, \quad \lambda_{51}=83.183, \quad \lambda_{52}=85.164, \quad \lambda_{53}=87.147, \quad \lambda_{54}=89.131 \text {, } \\
& \lambda_{55}=91.117, \quad \lambda_{56}=93.105, \quad \lambda_{57}=95.094, \quad \lambda_{58}=97.084 \text {, }
\end{aligned}
$$

and by virtue of the preliminary exponent

$$
\sigma(19)=0.00478 \quad(s=50, t=13, w=28),
$$

we have also

$$
\lambda_{s}=\max \{2 s-19,97.084+2(s-58)(1-0.00478)\}
$$

for $s>58$. Equipped with these preliminary bounds, we refine our procedure as indicated in the table. One may computationally check the validity of the appropriate case of Lemma 5.7 as follows.

(a) $45 \leq s \leq 47$. With process $N_{16}^{s}$, one finds that Lemma $5.7\left(\mathrm{I}^{\prime}\right)$ holds with $u=s-17$, by virtue of condition $\left(\mathrm{A}_{1}\right)$.

(b) $48 \leq s \leq 52$. With process $N_{15}^{s}$, one finds that Lemma 5.7( $\left(\mathrm{I}^{\prime}\right)$ holds with $u=s-16$, by virtue of condition $\left(\mathrm{A}_{1}\right)$.

(c) $53 \leq s \leq 58$. With process $N_{14}^{s}$, one finds that Lemma $5.7\left(\mathrm{I}^{\prime}\right)$ holds with $u=s-15$, by virtue of condition $\left(\mathrm{A}_{1}\right)$.

(d) $s \geq 59$. One finds that process $D^{s}$ applies.

22. Permissible exponents for twentieth powers. Following the computational procedure outlined in Section 8, we obtain the permissible exponents recorded in the table below. The exponents $\lambda_{s}$ listed in the table improve on those previously available for $s \geq 3$. We follow a similar path to that taken in previous sections. Note first that for $3 \leq s \leq 23$, the condition $(\delta)$ of Theorem 3.4 is satisfied. Since one of the conditions (i) and (iii) of Theorem 3.4 is also satisfied in this range of $s$, we deduce that one may take $\tau_{j, l}=0(l=1,2)$ for $3 \leq s \leq 23$. When $10 \leq j \leq 14$ and $24 \leq s \leq 33$, meanwhile, we must resort to Theorem $3.4(\mathrm{II})(1)$ and (2). Here we note 
that condition (iii) is satisfied for $24 \leq s \leq 30$, and that (II)(2) applies for $s=31,32,33$, and thus the estimate (4.1) holds for $10 \leq j \leq 14$ and $l=2,3$ with $\chi_{j, l}=1 / 3$ and $\tau_{j, l}=1$. Note here that when $s=31,32,33$, the relevant value of $\sigma$ is so small that the condition $\phi_{1}+\ldots+\phi_{j} \geq \frac{1}{3}(1-\sigma)^{-1}$ is satisfied transparently.

In order to discuss permissible exponents for $34 \leq s \leq 47$, we apply case (Ic) of Theorem 3.4. In the notation of the latter theorem, we find from Section 23 that when $j=15$ and $l=2$, one has $J=2$, and hence one may take

$$
\delta_{7}=0.0332148, \quad \delta_{8}=0.0661378, \quad \delta_{16}=0.6484502,
$$

whence we may take

$$
\tau_{15, l}=\frac{1}{15}\left(\delta_{7}+\delta_{8}\right)<0.00663 \quad \text { whenever } \quad \phi_{1} \leq 0.048742 \quad(l=2) .
$$

Further, when $j=15,16$ and $18 \leq j+l \leq 19$, one has $J=1$, and hence one may take

$$
\delta_{7}=0.1411109, \quad \delta_{8}=0.2285099, \quad \delta_{15}=1.2643687, \quad \delta_{16}=1.4713863 .
$$

Thus we deduce that one may take

$$
\tau_{15, l}=\frac{1}{15}\left(\delta_{7}+\delta_{8}\right)<0.02465 \quad \text { whenever } \quad \phi_{1} \leq 0.047586 \quad(l=3),
$$

and otherwise

and that one may take

$$
\tau_{15, l}=\frac{1}{15} \delta_{15}<0.08430 \quad(l=2,3),
$$

$$
\tau_{16, l}=\frac{1}{16} \delta_{16}<0.09197 \quad(l=2,3) .
$$

As in the previous cases, our computations for $s \geq 48$ depend on first obtaining preliminary estimates by applying the process $M_{14}^{s}$ for $48 \leq s \leq 61$ (noting (5.27) and checking $\left(\mathrm{D}_{1}\right)$ or $\left(\mathrm{D}_{2}\right)$ ), and $D^{s}$ for $s \geq 62$. In this way we obtain the preliminary permissible exponents

$$
\begin{aligned}
& \lambda_{48}=76.356, \quad \lambda_{49}=78.320, \quad \lambda_{50}=80.289, \quad \lambda_{51}=82.260, \quad \lambda_{52}=84.234, \\
& \lambda_{53}=86.211, \quad \lambda_{54}=88.190, \quad \lambda_{55}=90.171, \quad \lambda_{56}=92.154, \quad \lambda_{57}=94.138, \\
& \lambda_{58}=96.124, \quad \lambda_{59}=98.112, \quad \lambda_{60}=100.101, \quad \lambda_{61}=102.091 \text {, }
\end{aligned}
$$

and by virtue of the preliminary exponent

$$
\sigma(20)=0.00450 \quad(s=54, t=14, w=29),
$$

we have also

$$
\lambda_{s}=\max \{2 s-20,102.091+2(s-61)(1-0.00450)\}
$$

for $s>61$. Equipped with these preliminary bounds, we refine our procedure as indicated in the table. One may computationally check the validity of the appropriate case of Lemma 5.7 as follows. 
Table of permissible exponents for $k=20$

\begin{tabular}{|c|c|c|c|c|c|c|}
\hline$s$ & Process & $\lambda_{s}$ & $\phi_{1}$ & $\phi_{j}$ & $\sum_{i=1}^{j} \phi_{i}$ & $\phi_{s}^{*}$ \\
\hline 3 & $B_{1,4}^{3,2}$ & 3.0035377 & 0.00176883 & & & \\
\hline 4 & $B_{1,6}^{4,2}$ & 4.0372112 & 0.01123775 & & & \\
\hline 5 & $B_{1,6}^{5,2}$ & 5.1214726 & 0.02126317 & & & \\
\hline 6 & $A_{1}^{6,2}$ & 6.2457501 & 0.02547440 & & & \\
\hline 7 & $B_{2,9}^{7,2}$ & 7.4042791 & 0.02754990 & 0.0141 & 0.0417 & \\
\hline 8 & $A_{2}^{8,2}$ & 8.5946271 & 0.02885933 & 0.0215 & 0.0504 & \\
\hline 9 & $A_{2}^{9,2}$ & 9.8176862 & 0.03012126 & 0.0263 & 0.0564 & \\
\hline 0 & $B_{3,10}^{10,2}$ & 11.0720258 & 0.03108406 & 0.0183 & 0.0781 & 1.0176 \\
\hline 1 & $A_{3}^{11,2}$ & 12.3569582 & 0.03191458 & 0.0241 & 0.0862 & 1.1705 \\
\hline 2 & $B_{4,12}^{12,2}$ & 13.6733507 & 0.03281045 & 0.0162 & 0.1093 & 1.2987 \\
\hline 13 & $A_{4}^{13,2}$ & 15.0199527 & 0.03356385 & 0.0225 & 0.1189 & 1.3522 \\
\hline 4 & $B_{5,13}^{14,2}$ & 16.3974554 & 0.03438079 & 0.0150 & & 1.4113 \\
\hline 5 & $A_{5}^{15,2}$ & 17.8050250 & 0.03512761 & 0.0221 & 0.1551 & 1.4486 \\
\hline 6 & $B_{6,14}^{16,2}$ & 19.2428091 & 0.03589874 & 0.0148 & 0.1814 & 1.4887 \\
\hline 7 & $A_{6}^{17,2}$ & 20.7101660 & 0.03663479 & 0.0221 & 0.1941 & 1.5221 \\
\hline 8 & $A_{6}^{18,2}$ & 22.2068593 & 0.03737393 & 0.0293 & 0.2069 & 1.5565 \\
\hline 9 & $A_{7}^{19,2}$ & 23.7322237 & 0.03808882 & 0.0225 & 0.2356 & 1.5891 \\
\hline 20 & $A_{8}^{20,2}$ & 25.2857385 & 0.03879475 & 0.0159 & 0.2652 & 1.6243 \\
\hline 21 & $A_{8}^{21,2}$ & 26.8666319 & 0.03947826 & 0.0234 & 0.2799 & 1.6577 \\
\hline 22 & $A_{9}^{22,2}$ & 28.4741610 & 0.04014501 & 0.0169 & 0.3107 & 1.6935 \\
\hline 33 & $A_{9}^{23,2}$ & 30.1074344 & 0.04078835 & 0.0244 & 0.3260 & 1.7277 \\
\hline 24 & $A_{10}^{24,2}$ & 31.7655438 & 0.04140990 & 0.0182 & 0.3583 & 1.7639 \\
\hline 25 & $A_{10}^{25,2}$ & 33.4474901 & 0.04200611 & 0.0258 & 0.3745 & 1.7992 \\
\hline 26 & $A_{11}^{26,2}$ & 35.1522491 & 0.04257718 & & & 1.8352 \\
\hline 27 & $B_{11,21}^{27,3}$ & 36.8787481 & 0.04312142 & 0.0271 & 0.4245 & 1.8717 \\
\hline 8 & $A_{12}^{28,2}$ & 38.6258968 & 0.04363868 & 0.0210 & 0.4587 & 1.9073 \\
\hline 29 & $B_{12,22}^{29,3}$ & 40.3925867 & 0.04412832 & 0.0279 & 0.4751 & 1.9443 \\
\hline 30 & $A_{13}^{30,2}$ & 42.1777082 & 0.04459040 & 0.0216 & 0.5097 & 1.9795 \\
\hline 1 & $A_{13}^{31,2}$ & 43.9801557 & 0.04502494 & 0.0282 & 0.5259 & 2.0165 \\
\hline 32 & $A_{14}^{32,2}$ & 45.7988398 & 0.04543236 & 0.0216 & 0.5606 & 2.0515 \\
\hline 33 & $B_{14,25}^{33,3}$ & 47.6326920 & 0.04581314 & 0.0276 & 0.5763 & 2.0876 \\
\hline 34 & $A_{15}^{34,2}$ & 49.4806744 & 0.04616803 & 0.0163 & 0.6042 & 2.1205 \\
\hline 35 & $A_{15}^{35,2}$ & 51.3417836 & 0.04649788 & 0.0215 & 0.6187 & 2.1507 \\
\hline 36 & $A_{15}^{36,2}$ & 53.2150565 & 0.04680367 & 0.0264 & 0.6325 & 2.1835 \\
\hline 37 & $B_{15,28}^{37,3}$ & 55.0995728 & 0.04708646 & 0.0308 & 0.6453 & 2.2165 \\
\hline
\end{tabular}


Table of permissible exponents for $k=20$ (cont.)

\begin{tabular}{|c|c|c|c|c|c|c|}
\hline$s$ & Process & $\lambda_{s}$ & $\phi_{1}$ & $\phi_{j}$ & $\sum_{i=1}^{j} \phi_{i}$ & $\phi_{s}^{*}$ \\
\hline 38 & $B_{15,28}^{38,3}$ & 56.9944581 & 0.04734736 & 0.0339 & 0.6559 & 2.2467 \\
\hline 39 & $A_{16}^{39,2}$ & 58.8988854 & 0.04758756 & 0.0276 & 0.6924 & 2.2796 \\
\hline 40 & $B_{16,30}^{40,3}$ & 60.8120763 & 0.04780826 & 0.0312 & 0.7037 & 2.3097 \\
\hline 41 & $B_{16,30}^{41,3}$ & 62.7333008 & 0.04801065 & 0.0337 & 0.7130 & 2.3370 \\
\hline 42 & $A_{16}^{42,3}$ & 64.6618772 & 0.04819593 & 0.0356 & 0.7208 & 2.3636 \\
\hline 43 & $A_{16}^{43,3}$ & 66.5971707 & 0.04836528 & 0.0369 & 0.7276 & 2.3899 \\
\hline 44 & $A_{16}^{44,3}$ & 68.5385926 & 0.04851983 & 0.0381 & 0.7338 & 2.4148 \\
\hline 45 & $A_{16}^{45,3}$ & 70.4855980 & 0.04866069 & 0.0393 & 0.7397 & 2.4384 \\
\hline 46 & $A_{16}^{46,3}$ & 72.4376841 & 0.04878890 & 0.0404 & 0.7451 & 2.4606 \\
\hline 47 & $A_{16}^{47,3}$ & 74.3943883 & 0.04890547 & 0.0414 & 0.7502 & 2.4837 \\
\hline 48 & $N_{17}^{48}$ & 76.3552854 & 0.04901133 & 0.0375 & 0.7919 & 2.5047 \\
\hline 49 & $N_{17}^{49}$ & 78.3199857 & 0.04910737 & 0.0375 & 0.7949 & 2.5178 \\
\hline 50 & $N_{16}^{50}$ & 80.2881335 & 0.04919448 & 0.0435 & 0.7623 & 2.5464 \\
\hline 51 & $N_{16}^{51}$ & 82.2594024 & 0.04927331 & 0.0435 & 0.7647 & 2.5556 \\
\hline 52 & $N_{16}^{52}$ & 84.2334960 & 0.04934469 & 0.0435 & 0.7668 & 2.5640 \\
\hline 53 & $N_{16}^{53}$ & 86.2101439 & 0.04940924 & 0.0435 & 0.7687 & 2.5717 \\
\hline 54 & $N_{16}^{54}$ & 88.1891004 & 0.04946760 & 0.0435 & 0.7704 & 2.5787 \\
\hline 55 & $N_{16}^{55}$ & 90.1701423 & 0.04952031 & 0.0435 & 0.7720 & 2.5851 \\
\hline 56 & $N_{16}^{56}$ & 92.1530669 & 0.04956792 & 0.0435 & 0.7735 & 2.5909 \\
\hline 57 & $N_{15}^{57}$ & 94.1376919 & 0.04961094 & 0.0468 & 0.7328 & 2.6199 \\
\hline 58 & $N_{15}^{58}$ & 96.1238487 & 0.04964967 & 0.0468 & 0.7338 & 2.6224 \\
\hline 59 & $N_{15}^{59}$ & 98.1113875 & 0.04968462 & 0.0468 & 0.7348 & 2.6246 \\
\hline 60 & $N_{15}^{60}$ & 100.1001722 & 0.04971613 & 0.0468 & 0.7356 & 2.6266 \\
\hline 61 & $N_{15}^{61}$ & 102.0900795 & 0.04974452 & 0.0468 & 0.7364 & 2.6284 \\
\hline 62 & $N_{15}^{62}$ & 104.0809983 & 0.04977011 & 0.0468 & 0.7371 & 2.6301 \\
\hline$s \geq 63$ & $D^{s}$ & & & & & \\
\hline & ( & & & & $(v=62)$ & \\
\hline
\end{tabular}

(a) $s=48,49$. With process $N_{17}^{s}$, one finds that Lemma $5.7\left(\mathrm{I}^{\prime}\right)$ holds with $u=s-18$, by virtue of condition $\left(\mathrm{A}_{1}\right)$.

(b) $50 \leq s \leq 56$. With process $N_{16}^{s}$, one finds that Lemma $5.7\left(\mathrm{I}^{\prime}\right)$ holds with $u=s-17$, by virtue of condition $\left(\mathrm{A}_{1}\right)$.

(c) $57 \leq s \leq 62$. With process $N_{15}^{s}$, one finds that Lemma $5.7\left(\mathrm{I}^{\prime}\right)$ holds with $u=s-16$, by virtue of condition $\left(\mathrm{A}_{1}\right)$.

(d) $s \geq 63$. One finds that process $D^{s}$ applies. 
23. Auxiliary permissible exponents. We collect together in this section the permissible exponents for larger $k$ required in our calculation of the exponents in Sections 13-22. We do not work hard here to establish the sharpest such exponents, since good approximations will suffice.

Table of permissible exponents for $k=22$

\begin{tabular}{ccccccc}
\hline$s$ & Process & $\lambda_{s}$ & $\phi_{1}$ & $\phi_{j}$ & $\sum_{i=1}^{j} \phi_{i}$ & $\phi_{s}^{*}$ \\
\hline 3 & $B_{1,4}^{3,2}$ & 3.0025439 & 0.00127191 & & & \\
4 & $B_{1,2}^{4,6}$ & 4.0292912 & 0.00892335 & & & \\
5 & $B_{1,6}^{5,2}$ & 5.1025520 & 0.01845031 & & & \\
6 & $A_{1}^{6,2}$ & 6.2121725 & 0.02238321 & & & \\
7 & $B_{2,9}^{7,2}$ & 7.3545751 & 0.02460381 & 0.0104 & 0.0350 & \\
8 & $B_{2,9}^{8,2}$ & 8.5257736 & 0.02576186 & 0.0183 & 0.0440 & \\
9 & $A_{2}^{9,2}$ & 9.7256814 & 0.02674629 & 0.0225 & 0.0492 & \\
10 & $B_{3,10}^{10,2}$ & 10.9550112 & 0.02771586 & 0.0146 & 0.0678 & 0.9494 \\
\hline
\end{tabular}

Table of permissible exponents for $k=24$

\begin{tabular}{ccccccc}
\hline$s$ & Process & $\lambda_{s}$ & $\phi_{1}$ & $\phi_{j}$ & $\sum_{i=1}^{j} \phi_{i}$ & $\phi_{s}^{*}$ \\
\hline 3 & $B_{1,4}^{3,2}$ & 3.0018708 & 0.00093536 & & & \\
4 & $B_{1,2}^{4,6}$ & 4.0234059 & 0.00718285 & & & \\
5 & $B_{1,7}^{5,2}$ & 5.0866022 & 0.01589207 & & & \\
6 & $A_{1}^{6,2}$ & 6.1846118 & 0.01994744 & & & \\
7 & $B_{2,10}^{7,2}$ & 7.3136219 & 0.02218427 & 0.0071 & 0.0293 & \\
8 & $B_{2,9}^{8,2}$ & 8.4689321 & 0.02322785 & 0.0154 & 0.0386 & \\
9 & $A_{2}^{9,2}$ & 9.6501924 & 0.02406834 & 0.0195 & 0.0436 & \\
10 & $B_{3,11}^{10,2}$ & 10.8586937 & 0.02497080 & 0.0108 & 0.0586 & 0.8645 \\
11 & $B_{3,11}^{1,2}$ & 12.0918680 & 0.02550777 & 0.0167 & 0.0662 & 1.0427 \\
\hline & & & & & & \\
\hline
\end{tabular}

Table of permissible exponents for $k=26$

\begin{tabular}{|c|c|c|c|c|c|}
\hline$s$ & Process & $\lambda_{s}$ & $\phi_{1}$ & $\phi_{j}$ & $\sum_{i=1}^{j} \phi_{i} \quad \phi_{s}^{*}$ \\
\hline 3 & $B_{1,4}^{3,2}$ & 3.0014072 & 0.00070358 & & \\
\hline 4 & $B_{1,6}^{4,2}$ & 4.0190100 & 0.00587034 & & \\
\hline 5 & $B_{1,7}^{5,2}$ & 5.0738636 & 0.01377891 & & \\
\hline 6 & $B_{1,7}^{6,2}$ & 6.1622494 & 0.01794221 & & \\
\hline 7 & $B_{2,10}^{7,2}$ & 7.2802564 & 0.02021446 & 0.0045 & 0.0248 \\
\hline 8 & $B_{2,10}^{8,2}$ & 8.4222375 & 0.02112895 & 0.0129 & 0.0341 \\
\hline
\end{tabular}


Table of permissible exponents for $k=26$ (cont.)

\begin{tabular}{ccccccc}
\hline$s$ & Process & \multicolumn{1}{c}{$\lambda_{s}$} & $\phi_{1}$ & $\phi_{j}$ & $\sum_{i=1}^{j} \phi_{i}$ & $\phi_{s}^{*}$ \\
\hline 9 & $A_{2}^{9,2}$ & 9.5880882 & 0.02188651 & 0.0171 & 0.0390 & \\
10 & $B_{3,11}^{10,2}$ & 10.7792591 & 0.02272621 & 0.0082 & 0.0516 & 0.8018 \\
11 & $B_{3,11}^{11,2}$ & 11.9929177 & 0.02317152 & 0.0141 & 0.0591 & 0.9909 \\
\hline
\end{tabular}

Table of permissible exponents for $k=28$

\begin{tabular}{ccccccc}
\hline$s$ & Process & \multicolumn{1}{c}{$\lambda_{s}$} & $\phi_{1}$ & $\phi_{j}$ & $\sum_{i=1}^{j} \phi_{i}$ & $\phi_{s}^{*}$ \\
\hline 3 & $B_{1,4}^{3,2}$ & 3.0010768 & 0.00053837 & & & \\
4 & $B_{1,6}^{4,2}$ & 4.0156211 & 0.00484986 & & & \\
5 & $B_{1,8}^{5,2}$ & 5.0633584 & 0.01198110 & & & \\
6 & $B_{1,7}^{6,2}$ & 6.1434849 & 0.01623098 & & & \\
7 & $B_{2,10}^{7,2}$ & 7.2523170 & 0.01858310 & 0.0026 & 0.0212 & \\
8 & $B_{2,10}^{8,2}$ & 8.3829073 & 0.01935336 & 0.0107 & 0.0301 & \\
9 & $B_{2,10}^{9,2}$ & 9.5356712 & 0.02005540 & 0.0151 & 0.0351 & \\
10 & $A_{2}^{10,2}$ & 10.7106189 & 0.02066883 & 0.0179 & 0.0385 & \\
11 & $B_{3,12}^{11,2}$ & 11.9079284 & 0.02124033 & 0.0115 & 0.0526 & 0.9281 \\
12 & $A_{3}^{12,2}$ & 13.1262695 & 0.02163492 & 0.0154 & 0.0576 & 1.0629 \\
\hline
\end{tabular}

Table of permissible exponents for $k=30$

\begin{tabular}{ccccccc}
\hline$s$ & Process & \multicolumn{1}{c}{$\lambda_{s}$} & $\phi_{1}$ & $\phi_{j}$ & $\sum_{i=1}^{j} \phi_{i}$ & $\phi_{s}^{*}$ \\
\hline 3 & $B_{1,4}^{3,2}$ & 3.0008289 & 0.00041441 & & & \\
4 & $B_{1,6}^{4,2}$ & 4.0128519 & 0.00400878 & & & \\
5 & $B_{1,8}^{5,2}$ & 5.0536213 & 0.01022522 & & & \\
6 & $B_{1,7}^{6,2}$ & 6.1264298 & 0.01471957 & & & \\
7 & $A_{1}^{7,2}$ & 7.2247478 & 0.01673904 & & & \\
8 & $B_{2,11}^{8,2}$ & 8.3459599 & 0.01789043 & 0.0087 & 0.0266 & \\
9 & $B_{2,10}^{9,2}$ & 9.4875943 & 0.01850453 & 0.0134 & 0.0319 & \\
10 & $A_{2}^{10,2}$ & 10.6496179 & 0.01903383 & 0.0160 & 0.0350 & \\
11 & $B_{3,13}^{11,2}$ & 11.8328152 & 0.01959249 & 0.0094 & 0.0472 & 0.8726 \\
12 & $A_{3}^{12,2}$ & 13.0354619 & 0.01993146 & 0.0135 & 0.0523 & 1.0225 \\
13 & $B_{4,14}^{13,2}$ & 14.2585847 & 0.02034949 & 0.0061 & 0.0641 & 1.1865 \\
14 & $B_{4,14}^{14,2}$ & 15.5012985 & 0.02067161 & 0.0113 & 0.0708 & 1.2261 \\
\hline & & & & & &
\end{tabular}


Table of permissible exponents for $k=32$

\begin{tabular}{ccccccc}
\hline$s$ & Process & \multicolumn{1}{c}{$\lambda_{s}$} & $\phi_{1}$ & $\phi_{j}$ & $\sum_{i=1}^{j} \phi_{i}$ & $\phi_{s}^{*}$ \\
\hline 3 & $B_{1,4}^{3,2}$ & 3.0006513 & 0.00032563 & & & \\
4 & $B_{1,6}^{4,2}$ & 4.0107492 & 0.00336671 & & & \\
5 & $B_{1,2}^{5,2}$ & 5.0460456 & 0.00884787 & & & \\
6 & $B_{1,8}^{6,2}$ & 6.1127790 & 0.01347074 & & & \\
7 & $A_{1}^{7,2}$ & 7.2031814 & 0.01535571 & & & \\
8 & $B_{2,11}^{8,2}$ & 8.3160843 & 0.01661114 & 0.0070 & 0.0236 & \\
9 & $B_{2,11}^{9,2}$ & 9.4480157 & 0.01716982 & 0.0118 & 0.0290 & \\
10 & $B_{2,11}^{10,2}$ & 10.5989372 & 0.01764754 & 0.0145 & 0.0321 & \\
11 & $A_{2}^{11,2}$ & 11.7694872 & 0.01814158 & 0.0166 & 0.0348 & \\
12 & $B_{3,13}^{12,2}$ & 12.9585035 & 0.01847574 & 0.0117 & 0.0477 & 0.9802 \\
13 & $A_{3}^{13,2}$ & 14.1659645 & 0.01878922 & 0.0145 & 0.0513 & 1.0906 \\
14 & $B_{4,15}^{14,2}$ & 15.3924025 & 0.01913448 & 0.0091 & 0.0641 & 1.2034 \\
15 & $A_{4}^{15,2}$ & 16.6372483 & 0.01942050 & 0.0127 & 0.0690 & 1.2378 \\
\hline
\end{tabular}

Table of permissible exponents for $k=34$

\begin{tabular}{ccccccc}
\hline$s$ & Process & \multicolumn{1}{c}{$\lambda_{s}$} & $\phi_{1}$ & $\phi_{j}$ & $\sum_{i=1}^{j} \phi_{i}$ & $\phi_{s}^{*}$ \\
\hline 3 & $B_{1,4}^{3,2}$ & 3.0005147 & 0.00025732 & & & \\
4 & $B_{1,2}^{4,6}$ & 4.0090083 & 0.00283170 & & & \\
5 & $B_{1,2}^{5,2}$ & 5.0397401 & 0.00770030 & & & \\
6 & $B_{1,2}^{6,2}$ & 6.1004200 & 0.01223322 & & & \\
7 & $A_{1}^{7,2}$ & 7.1840767 & 0.01418011 & & & \\
8 & $B_{2,12}^{8,2}$ & 8.2896184 & 0.01548459 & 0.0054 & 0.0209 & \\
9 & $B_{2,11}^{9,2}$ & 9.4129882 & 0.01600048 & 0.0104 & 0.0264 & \\
10 & $B_{2,11}^{10,2}$ & 10.5541305 & 0.01643672 & 0.0131 & 0.0295 & \\
11 & $A_{2}^{11,2}$ & 11.7133896 & 0.01686019 & 0.0150 & 0.0319 & \\
12 & $B_{3,13}^{12,2}$ & 12.8905163 & 0.01721916 & 0.0101 & 0.0436 & 0.9376 \\
13 & $A_{3}^{13,2}$ & 14.0847742 & 0.01748578 & 0.0129 & 0.0471 & 1.0521 \\
14 & $A_{3}^{14,2}$ & 15.2966345 & 0.01778064 & 0.0154 & 0.0504 & 1.1577 \\
15 & $B_{4,15}^{15,2}$ & 16.5259780 & 0.01805376 & 0.0109 & 0.0632 & 1.2170 \\
\hline
\end{tabular}


Table of permissible exponents for $k=36$

\begin{tabular}{ccccccc}
\hline$s$ & Process & $\lambda_{s}$ & $\phi_{1}$ & $\phi_{j}$ & $\sum_{i=1}^{j} \phi_{i}$ & $\phi_{s}^{*}$ \\
\hline 3 & $B_{1,4}^{3,2}$ & 3.0004128 & 0.00020635 & & & \\
4 & $B_{1,2}^{4,6}$ & 4.0076365 & 0.00240826 & & & \\
5 & $B_{1,2}^{5,2}$ & 5.0346195 & 0.00675866 & & & \\
6 & $B_{1,2}^{6,2}$ & 6.0901459 & 0.01118271 & & & \\
7 & $A_{1}^{7,2}$ & 7.1679365 & 0.01316287 & & & \\
8 & $B_{2,12}^{8,2}$ & 8.2670449 & 0.01450637 & 0.0040 & 0.0185 & \\
9 & $B_{2,12}^{9,2}$ & 9.3828824 & 0.01497972 & 0.0091 & 0.0241 & \\
10 & $B_{2,11}^{10,2}$ & 10.5154507 & 0.01538430 & 0.0118 & 0.0272 & \\
11 & $A_{2}^{11,2}$ & 11.6648323 & 0.01575000 & 0.0137 & 0.0294 & \\
12 & $B_{3,14}^{12,2}$ & 12.8314116 & 0.01611772 & 0.0086 & 0.0398 & 0.8917 \\
13 & $A_{3}^{13,2}$ & 14.0140325 & 0.01635130 & 0.0115 & 0.0435 & 1.0180 \\
14 & $A_{3}^{14,2}$ & 15.2130601 & 0.01660506 & 0.0137 & 0.0464 & 1.1166 \\
15 & $B_{4,16}^{15,2}$ & 16.4286845 & 0.01686286 & 0.0093 & 0.0581 & 1.1986 \\
16 & $A_{4}^{16,2}$ & 17.6606068 & 0.01708916 & 0.0120 & 0.0618 & 1.2277 \\
\hline & & & & & &
\end{tabular}

Table of permissible exponents for $k=38$

\begin{tabular}{ccccccc}
\hline$s$ & Process & $\lambda_{s}$ & $\phi_{1}$ & $\phi_{j}$ & $\sum_{i=1}^{j} \phi_{i}$ & $\phi_{s}^{*}$ \\
\hline 3 & $B_{1,4}^{3,2}$ & 3.0003353 & 0.00016763 & & & \\
4 & $B_{1,2}^{4,2}$ & 4.0065387 & 0.00206802 & & & \\
5 & $B_{1,2}^{5,2}$ & 5.0304085 & 0.00597725 & & & \\
6 & $B_{1,2}^{6,2}$ & 6.0814901 & 0.01027884 & & & \\
7 & $A_{1}^{7,2}$ & 7.1541435 & 0.01227563 & & & \\
8 & $B_{2,12}^{8,2}$ & 8.2475913 & 0.01365028 & 0.0029 & 0.0166 & \\
9 & $B_{2,12}^{9,2}$ & 9.3566575 & 0.01406869 & 0.0079 & 0.0220 & \\
10 & $B_{2,11}^{10,2}$ & 10.4816593 & 0.01446221 & 0.0108 & 0.0253 & \\
11 & $A_{2}^{11,2}$ & 11.6223319 & 0.01477912 & 0.0125 & 0.0273 & \\
12 & $B_{3,14}^{12,2}$ & 12.7795262 & 0.01514737 & 0.0072 & 0.0366 & 0.8484 \\
13 & $B_{3,14}^{13,2}$ & 13.9517878 & 0.01535244 & 0.0102 & 0.0402 & 0.9829 \\
14 & $A_{3}^{14,2}$ & 15.1394461 & 0.01557562 & 0.0123 & 0.0429 & 1.0800 \\
15 & $A_{3}^{15,2}$ & 16.3428361 & 0.01581504 & 0.0144 & 0.0457 & 1.1800 \\
16 & $A_{4}^{16,2}$ & 17.5615789 & 0.01601671 & 0.0105 & 0.0571 & 1.2100 \\
\hline
\end{tabular}


Table of permissible exponents for $k=40$

\begin{tabular}{ccccccc}
\hline$s$ & Process & $\lambda_{s}$ & $\phi_{1}$ & $\phi_{j}$ & $\sum_{i=1}^{j} \phi_{i}$ & $\phi_{s}^{*}$ \\
\hline 3 & $B_{1,4}^{3,2}$ & 3.0002717 & 0.00013584 & & & \\
4 & $B_{1,2}^{4,6}$ & 4.0055702 & 0.00176633 & & & \\
5 & $B_{1,2}^{5,2}$ & 5.0266358 & 0.00527374 & & & \\
6 & $B_{1,9}^{6,2}$ & 6.0730909 & 0.00934078 & & & \\
7 & $A_{1}^{7,2}$ & 7.1411109 & 0.01147649 & & & \\
8 & $A_{1}^{8,2}$ & 8.2285099 & 0.01274245 & & & \\
9 & $B_{2,13}^{9,2}$ & 9.3316986 & 0.01327785 & 0.0069 & 0.0201 & \\
10 & $B_{2,12}^{10,2}$ & 10.4498752 & 0.01363320 & 0.0098 & 0.0235 & \\
11 & $A_{2}^{11,2}$ & 11.5828707 & 0.01392605 & 0.0115 & 0.0255 & \\
12 & $A_{2}^{12,2}$ & 12.7314053 & 0.01425870 & 0.0132 & 0.0274 & \\
13 & $B_{3,14}^{13,2}$ & 13.8944894 & 0.01447244 & 0.0091 & 0.0374 & 0.9509 \\
14 & $A_{3}^{14,2}$ & 15.0720426 & 0.01466715 & 0.0111 & 0.0399 & 1.0482 \\
15 & $A_{3}^{15,2}$ & 16.2643687 & 0.01487677 & 0.0130 & 0.0424 & 1.1421 \\
16 & $A_{4}^{16,2}$ & 17.4713863 & 0.01507158 & 0.0093 & 0.0531 & 1.1947 \\
17 & $A_{5}^{17,2}$ & 18.6931953 & 0.01526705 & 0.0056 & 0.0641 & 1.2358 \\
\hline
\end{tabular}

Table of permissible exponents for $k=56$

\begin{tabular}{ccccccc}
\hline$s$ & Process & $\lambda_{s}$ & $\phi_{1}$ & $\phi_{j}$ & $\sum_{i=1}^{j} \phi_{i}$ & $\phi_{s}^{*}$ \\
\hline 3 & $B_{1,4}^{3,2}$ & 3.0000641 & 0.00003202 & & & \\
4 & $B_{1,2}^{4,2}$ & 4.0018248 & 0.00058692 & & & \\
5 & $B_{1,2}^{5,2}$ & 5.0107720 & 0.00223784 & & & \\
6 & $B_{1,10}^{6,2}$ & 6.0336021 & 0.00457587 & & & \\
7 & $B_{1,10}^{7,2}$ & 7.0749443 & 0.00692919 & & & \\
8 & $B_{1,10}^{8,2}$ & 8.1314920 & 0.00816567 & & & \\
9 & $A_{1}^{9,2}$ & 9.2015653 & 0.00890554 & & & \\
10 & $B_{2,15}^{10,2}$ & 10.2835673 & 0.00932007 & 0.0041 & 0.0134 & \\
11 & $B_{2,14}^{11,2}$ & 11.3759867 & 0.00951166 & 0.0063 & 0.0158 & \\
12 & $B_{2,14}^{12,2}$ & 12.4787878 & 0.00967630 & 0.0076 & 0.0173 & \\
13 & $A_{2}^{13,2}$ & 13.5920033 & 0.00982670 & 0.0086 & 0.0184 & \\
14 & $A_{2}^{14,2}$ & 14.7160440 & 0.00999685 & 0.0095 & 0.0195 & \\
15 & $A_{3}^{15,2}$ & 15.8501204 & 0.01009311 & 0.0067 & 0.0265 & 0.9447 \\
16 & $A_{4}^{16,2}$ & 16.9946507 & 0.01021425 & 0.0040 & 0.0337 & 1.1211 \\
\hline & & & & & &
\end{tabular}


Table of permissible exponents for $k=60$

\begin{tabular}{ccccccc}
\hline$s$ & Process & $\lambda_{s}$ & $\phi_{1}$ & $\phi_{j}$ & $\sum_{i=1}^{j} \phi_{i}$ & $\phi_{s}^{*}$ \\
\hline 3 & $B_{1,4}^{3,2}$ & 3.0000471 & 0.00002352 & & & \\
4 & $B_{1,2}^{4,2}$ & 4.0014347 & 0.00046255 & & & \\
5 & $B_{1,8}^{5,2}$ & 5.0088563 & 0.00185607 & & & \\
6 & $B_{1,1}^{6,2}$ & 6.0283226 & 0.00390017 & & & \\
7 & $B_{1,1}^{7,2}$ & 7.0648348 & 0.00611424 & & & \\
8 & $B_{1,1}^{8,2}$ & 8.1162996 & 0.00742085 & & & \\
9 & $A_{1}^{9,2}$ & 9.1804196 & 0.00813325 & & & \\
10 & $B_{2,16}^{10,2}$ & 10.2565541 & 0.00863245 & 0.0031 & 0.0117 & \\
11 & $B_{2,15}^{11,2}$ & 11.3423160 & 0.00880201 & 0.0053 & 0.0142 & \\
12 & $B_{2,15}^{12,2}$ & 12.4377875 & 0.00895800 & 0.0068 & 0.0157 & \\
13 & $A_{2}^{13,2}$ & 13.5428594 & 0.00908753 & 0.0077 & 0.0167 & \\
14 & $A_{2}^{14,2}$ & 14.6578046 & 0.00922726 & 0.0085 & 0.0177 & \\
15 & $A_{3}^{15,2}$ & 15.7824957 & 0.00934563 & 0.0059 & 0.0242 & 0.9129 \\
16 & $A_{4}^{16,2}$ & 16.9168390 & 0.00944915 & 0.0033 & 0.0308 & 1.1104 \\
\hline
\end{tabular}

Table of permissible exponents for $k=64$

\begin{tabular}{ccccccc}
\hline$s$ & Process & $\lambda_{s}$ & $\phi_{1}$ & $\phi_{j}$ & $\sum_{i=1}^{j} \phi_{i}$ & $\phi_{s}^{*}$ \\
\hline 3 & $B_{1,4}^{3,2}$ & 3.0000353 & 0.00001762 & & & \\
4 & $B_{1,6}^{4,2}$ & 4.0011451 & 0.00036996 & & & \\
5 & $B_{1,2}^{5,2}$ & 5.0073658 & 0.00155562 & & & \\
6 & $B_{1,10}^{6,2}$ & 6.0241263 & 0.00335705 & & & \\
7 & $B_{1,11}^{7,2}$ & 7.0565053 & 0.00541830 & & & \\
8 & $B_{1,1}^{8,2}$ & 8.1035626 & 0.00677718 & & & \\
9 & $A_{1}^{9,2}$ & 9.1626169 & 0.00747861 & & & \\
10 & $B_{2,16}^{10,2}$ & 10.2336743 & 0.00804055 & 0.0022 & 0.0103 & \\
11 & $B_{2,16}^{11,2}$ & 11.3136439 & 0.00818830 & 0.0045 & 0.0127 & \\
12 & $B_{2,15}^{12,2}$ & 12.4026992 & 0.00833355 & 0.0060 & 0.0143 & \\
13 & $B_{2,15}^{13,2}$ & 13.5007324 & 0.00845312 & 0.0069 & 0.0153 & \\
14 & $A_{2}^{14,2}$ & 14.6078413 & 0.00856921 & 0.0076 & 0.0162 & \\
15 & $B_{3,18}^{15,2}$ & 15.7242889 & 0.00869521 & 0.0047 & 0.0216 & 0.8471 \\
16 & $B_{3,18}^{16,2}$ & 16.8494116 & 0.00876473 & 0.0059 & 0.0232 & 0.9414 \\
17 & $B_{4,18}^{17,2}$ & 17.9835998 & 0.00885697 & 0.0031 & 0.0289 & 1.1088 \\
18 & $B_{5,18}^{18,2}$ & 19.1266047 & 0.00892866 & 0.0004 & 0.0348 & 1.1504 \\
\hline & & & & & &
\end{tabular}


Table of permissible exponents for $k=68$

\begin{tabular}{ccccccc}
\hline$s$ & Process & $\lambda_{s}$ & $\phi_{1}$ & $\phi_{j}$ & $\sum_{i=1}^{j} \phi_{i}$ & $\phi_{s}^{*}$ \\
\hline 3 & $B_{1,4}^{3,2}$ & 3.0000267 & 0.00001331 & & & \\
4 & $B_{1,2}^{4,6}$ & 4.0009181 & 0.00029715 & & & \\
5 & $B_{1,2}^{5,2}$ & 5.0061304 & 0.00130338 & & & \\
6 & $B_{1,10}^{6,2}$ & 6.0205621 & 0.00288990 & & & \\
7 & $B_{1,12}^{7,2}$ & 7.0489837 & 0.00475323 & & & \\
8 & $B_{1,11}^{8,2}$ & 8.0918863 & 0.00617213 & & & \\
9 & $B_{1,11}^{9,2}$ & 9.1465073 & 0.00690696 & & & \\
10 & $A_{1}^{10,2}$ & 10.2122415 & 0.00742466 & & & \\
11 & $B_{2,17}^{11,2}$ & 11.2872180 & 0.00766025 & 0.0037 & 0.0114 & \\
12 & $B_{2,16}^{12,2}$ & 12.3706630 & 0.00778929 & 0.0053 & 0.0130 & \\
13 & $B_{2,15}^{13,2}$ & 13.4625637 & 0.00790250 & 0.0062 & 0.0142 & \\
14 & $A_{2}^{14,2}$ & 14.5628858 & 0.00800180 & 0.0069 & 0.0149 & \\
15 & $A_{2}^{15,2}$ & 15.6719470 & 0.00811643 & 0.0076 & 0.0158 & \\
16 & $B_{3,19}^{16,2}$ & 16.7893310 & 0.00819260 & 0.0051 & 0.0212 & 0.9024 \\
17 & $B_{3,19}^{17,2}$ & 17.9149233 & 0.00825686 & 0.0061 & 0.0224 & 0.9791 \\
18 & $A_{3}^{18,2}$ & 19.0488059 & 0.00832341 & 0.0069 & 0.0234 & 1.0441 \\
19 & $A_{4}^{19,2}$ & 20.1911221 & 0.00839565 & 0.0047 & 0.0293 & 1.1259 \\
20 & $A_{5}^{20,2}$ & 21.3417807 & 0.00845975 & 0.0024 & 0.0352 & 1.1581 \\
\hline & & & & & &
\end{tabular}

Table of permissible exponents for $k=72$

\begin{tabular}{|c|c|c|c|c|c|c|}
\hline$s$ & Process & $\lambda_{s}$ & $\phi_{1}$ & $\phi_{j}$ & $\sum_{i=1}^{j} \phi_{i}$ & $\phi_{s}^{*}$ \\
\hline 3 & $B_{1,4}^{3,2}$ & 3.0000205 & 0.00001021 & & & \\
\hline 4 & $B_{1,6}^{4,2}$ & 4.0007451 & 0.00024154 & & & \\
\hline 5 & $B_{1,8}^{5,2}$ & 5.0051551 & 0.00110272 & & & \\
\hline 6 & $B_{1,10}^{6,2}$ & 6.0176775 & 0.00250706 & & & \\
\hline 7 & $B_{1,12}^{7,2}$ & 7.0427686 & 0.00419421 & & & \\
\hline 8 & $B_{1,12}^{8,2}$ & 8.0820772 & 0.00565004 & & & \\
\hline 9 & $B_{1,11}^{9,2}$ & 9.1327314 & 0.00639741 & & & \\
\hline 10 & $A_{1}^{10,2}$ & 10.1937853 & 0.00688532 & & & \\
\hline 11 & $B_{2,18}^{11,2}$ & 11.2643373 & 0.00719463 & 0.0031 & 0.0102 & \\
\hline 12 & $B_{2,17}^{12,2}$ & 12.3428217 & 0.00731063 & 0.0046 & 0.0119 & \\
\hline 13 & $B_{2,16}^{13,2}$ & 13.4292736 & 0.00741620 & 0.0056 & 0.0131 & \\
\hline 14 & $A_{2}^{14,2}$ & 14.5236334 & 0.00750632 & 0.0063 & 0.0138 & \\
\hline
\end{tabular}


Table of permissible exponents for $k=72$ (cont.)

\begin{tabular}{ccccccc}
\hline$s$ & Process & $\lambda_{s}$ & $\phi_{1}$ & $\phi_{j}$ & $\sum_{i=1}^{j} \phi_{i}$ & $\phi_{s}^{*}$ \\
\hline 15 & $A_{2}^{15,2}$ & 15.6261004 & 0.00760346 & 0.0070 & 0.0146 & \\
16 & $B_{3,20}^{16,2}$ & 16.7366445 & 0.00769062 & 0.0044 & 0.0195 & 0.8628 \\
17 & $B_{3,19}^{17,2}$ & 17.8548813 & 0.00774646 & 0.0054 & 0.0207 & 0.9486 \\
18 & $A_{3}^{18,2}$ & 18.9808873 & 0.00780459 & 0.0062 & 0.0216 & 1.0116 \\
19 & $A_{3}^{19,2}$ & 20.1147519 & 0.00786555 & 0.0070 & 0.0226 & 1.0785 \\
20 & $B_{4,22}^{20,2}$ & 21.2564959 & 0.00792520 & 0.0048 & 0.0280 & 1.1276 \\
21 & $B_{5,22}^{21,2}$ & 22.4061171 & 0.00798257 & 0.0024 & 0.0334 & 1.1564 \\
22 & $A_{6}^{22,2}$ & 23.5636041 & 0.00803757 & 0.0006 & 0.0394 & 1.1688 \\
\hline
\end{tabular}

Table of permissible exponents for $k=76$

\begin{tabular}{ccccccc}
\hline$s$ & Process & $\lambda_{s}$ & $\phi_{1}$ & $\phi_{j}$ & $\sum_{i=1}^{j} \phi_{i}$ & $\phi_{s}^{*}$ \\
\hline 3 & $B_{1,4}^{3,2}$ & 3.0000159 & 0.00000793 & & & \\
4 & $B_{1,2}^{4,2}$ & 4.0006104 & 0.00019818 & & & \\
5 & $B_{1,2}^{5,2}$ & 5.0043590 & 0.00093729 & & & \\
6 & $B_{1,10}^{6,2}$ & 6.0152939 & 0.00218890 & & & \\
7 & $B_{1,12}^{7,2}$ & 7.0375713 & 0.00372240 & & & \\
8 & $B_{1,12}^{8,2}$ & 8.0734591 & 0.00515450 & & & \\
9 & $B_{1,11}^{9,2}$ & 9.1206671 & 0.00595570 & & & \\
10 & $A_{1}^{10,2}$ & 10.1776325 & 0.00641551 & & & \\
11 & $B_{2,1,2}^{11,2}$ & 11.2442251 & 0.00677969 & 0.0024 & 0.0092 & \\
12 & $B_{2,17}^{12,2}$ & 12.3182827 & 0.00688538 & 0.0040 & 0.0109 & \\
13 & $B_{2,17}^{13,2}$ & 13.3998892 & 0.00698584 & 0.0051 & 0.0121 & \\
14 & $A_{2}^{14,2}$ & 14.4889692 & 0.00706978 & 0.0058 & 0.0129 & \\
15 & $A_{2}^{15,2}$ & 15.5856100 & 0.00715274 & 0.0064 & 0.0135 & \\
16 & $B_{3,21}^{16,2}$ & 16.6900569 & 0.00724602 & 0.0037 & 0.0179 & 0.8224 \\
17 & $B_{3,20}^{17,2}$ & 17.8017421 & 0.00729495 & 0.0048 & 0.0192 & 0.9160 \\
18 & $A_{3}^{18,2}$ & 18.9207482 & 0.00734685 & 0.0056 & 0.0201 & 0.9828 \\
19 & $A_{3}^{19,2}$ & 20.0471373 & 0.00740016 & 0.0063 & 0.0209 & 1.0454 \\
20 & $A_{3}^{20,2}$ & 21.1809902 & 0.00745580 & 0.0070 & 0.0219 & 1.1119 \\
21 & $B_{4,23}^{21,2}$ & 22.3222235 & 0.00750483 & 0.0050 & 0.0271 & 1.1305 \\
22 & $B_{5,24}^{2,2}$ & 23.4709154 & 0.00755634 & 0.0026 & 0.0319 & 1.1551 \\
23 & $A_{5}^{23,2}$ & 24.6270386 & 0.00760498 & 0.0038 & 0.0335 & 1.1634 \\
24 & $A_{6}^{24,2}$ & 25.7906446 & 0.00765482 & 0.0018 & 0.0389 & 1.1744 \\
\hline & & & & & \\
& & & & \\
\end{tabular}


Table of permissible exponents for $k=80$

\begin{tabular}{|c|c|c|c|c|c|c|}
\hline$s$ & Process & $\lambda_{s}$ & $\phi_{1}$ & $\phi_{j}$ & $\sum_{i=1}^{j} \phi_{i}$ & $\phi_{s}^{*}$ \\
\hline 3 & $B_{1,4}^{3,2}$ & 3.0000125 & 0.00000624 & & & \\
\hline 4 & $B_{1,6}^{4,2}$ & 4.0005051 & 0.00016419 & & & \\
\hline 5 & $B_{1,8}^{5,2}$ & 5.0037183 & 0.00080341 & & & \\
\hline 6 & $B_{1,10}^{6,2}$ & 6.0133258 & 0.00192294 & & & \\
\hline 7 & $B_{1,12}^{7,2}$ & 7.0332148 & 0.00332221 & & & \\
\hline 8 & $B_{1,12}^{8,2}$ & 8.0661378 & 0.00472571 & & & \\
\hline 9 & $B_{1,12}^{9,2}$ & 9.1101284 & 0.00554468 & & & \\
\hline 10 & $A_{1}^{10,2}$ & 10.1634946 & 0.00600304 & & & \\
\hline 11 & $B_{2,18}^{11,2}$ & 11.2265639 & 0.00641176 & 0.0019 & 0.0083 & \\
\hline 12 & $B_{2,18}^{12,2}$ & 12.2966473 & 0.00650521 & 0.0035 & 0.0100 & \\
\hline 13 & $B_{2,17}^{13,2}$ & 13.3738933 & 0.00660034 & 0.0046 & 0.0112 & \\
\hline 14 & $B_{2,17}^{14,2}$ & 14.4582437 & 0.00668064 & 0.0053 & 0.0120 & \\
\hline 15 & $A_{2}^{15,2}$ & 15.5496954 & 0.00675332 & 0.0059 & 0.0126 & \\
\hline 16 & $A_{2}^{16,2}$ & 16.6484502 & 0.00683410 & 0.0064 & 0.0133 & \\
\hline 17 & $B_{3,21}^{17,2}$ & 17.7542883 & 0.00689430 & 0.0042 & 0.0178 & 0.8823 \\
\hline 18 & $A_{3}^{18,2}$ & 18.8670322 & 0.00693992 & 0.0050 & 0.0187 & 0.9570 \\
\hline 19 & $A_{3}^{19,2}$ & 19.9867390 & 0.00698693 & 0.0057 & 0.0195 & 1.0160 \\
\hline 20 & $A_{3}^{20,2}$ & 21.1134792 & 0.00703595 & 0.0063 & 0.0203 & 1.0785 \\
\hline 21 & $B_{4,24}^{21,2}$ & 22.2472590 & 0.00708335 & 0.0044 & 0.0252 & 1.1204 \\
\hline 22 & $B_{5,24}^{22,2}$ & 23.3880855 & 0.00712947 & 0.0020 & 0.0297 & 1.1467 \\
\hline 23 & $A_{5}^{23,2}$ & 24.5359375 & 0.00717314 & 0.0032 & 0.0312 & 1.1545 \\
\hline 24 & $A_{6}^{24,2}$ & 25.6908623 & 0.00721788 & 0.0013 & 0.0363 & 1.1651 \\
\hline
\end{tabular}

\section{References}

[1] R. C. Baker, Diophantine Inequalities, Clarendon Press, Oxford, 1986.

[2] E. Bombieri and J. Pila, The number of integral points on arcs and ovals, Duke Math. J. 59 (1989), 337-357.

[3] J. Brüdern, A problem in additive number theory, Math. Proc. Cambridge Philos. Soc. 103 (1988), 27-33.

[4] J. Brüdern and T. D. Wooley, On Waring's problem for cubes and smooth Weyl sums, Proc. London Math. Soc., to appear.

[5] I. Danicic, Contributions to Number Theory, Ph.D. thesis, London, 1957.

[6] T. Estermann, Einige Sätze über quadratfreie Zahlen, Math. Ann. 105 (1931), 653-662.

[7] D. R. Heath-Brown, On the fractional part of $\alpha n^{k}$, Mathematika 35 (1988), 28-37.

[8] H. Z. Li, Waring's problem for sixteenth powers, Sci. China Ser. A 39 (1996), 56-64. 
[9] Z. Z. Meng, Some new results in Waring's problem, Adv. in Math. (China) 25 (1996), 347-353.

[10] —, Some new results in Waring's problem, Acta Math. Sinica 12 (1996), 262-267.

[11] _, Some new results on Waring's problem, J. China Univ. Sci. Tech. 27 (1997), $1-5$.

[12] R. C. Vaughan, On Waring's problem for smaller exponents, Proc. London Math. Soc. (3) 52 (1986), 445-463.

[13] -, A new iterative method in Waring's problem, Acta Math. 162 (1989), 1-71.

[14] -, A new iterative method in Waring's problem, II, J. London Math. Soc. (2) 39 (1989), 219-230.

[15] - The Hardy-Littlewood Method, 2nd ed., Cambridge Univ. Press, Cambridge, 1997.

[16] R. C. Vaughan and T. D. Wooley, Further improvements in Waring's problem, III: Eighth powers, Philos. Trans. Roy. Soc. London Ser. A 345 (1993), 385-396.

[17] —, - Further improvements in Waring's problem, II: Sixth powers, Duke Math. J. 76 (1994), 683-710.

[18] -, -, Further improvements in Waring's problem, Acta Math. 174 (1995), 147-240.

[19] T. D. Wooley, Large improvements in Waring's problem, Ann. of Math. 135 (1992), $131-164$

[20] - , The application of a new mean value theorem to the fractional parts of polynomials, Acta Arith. 65 (1993), 163-179.

[21] —, Breaking classical convexity in Waring's problem: sums of cubes and quasidiagonal behaviour, Invent. Math. 122 (1995), 421-451.

[22] -, New estimates for smooth Weyl sums, J. London Math. Soc. (2) 51 (1995), 1-13.

Department of Mathematics

McAllister Building

Pennsylvania State University

University Park, PA 16802, U.S.A.

E-mail: rvaughan@math.psu.edu
Department of Mathematics University of Michigan East Hall

525 East University Avenue Ann Arbor, MI 48109-1109, U.S.A. E-mail: wooley@math.lsa.umich.edu 